\title{
Microfluidic surgery in single cells and multicellular systems
}

\author{
Kevin S. Zhang ${ }^{\dagger}$, Ambika V. Nadkarni ${ }^{\dagger}{ }^{\ddagger}, 1$, Rajorshi Paul ${ }^{\dagger, 1}$, Adrian M. Martin ${ }^{\dagger}, 1$, and Sindy K.Y. \\ $\operatorname{Tang}^{\dagger}, *$ \\ 'Department of Mechanical Engineering, Stanford University, Stanford, CA, 94305, USA \\ * Department of Biochemistry and Biophysics, University of California San Francisco, San Francisco, CA 94158, USA. \\ ${ }^{1}$ Equal contribution.
}

\begin{abstract}
Microscale surgery on single cells and small organisms have enabled major advances in fundamental biology and in engineering biological systems. Examples of applications range from wound healing and regeneration studies to the generation of hybridoma to produce monoclonal antibodies. Even today, these surgical operations are often performed manually, but they are labor-intensive and lack reproducibility. Microfluidics has emerged as a powerful technology to control and manipulate cells and multicellular systems at the micro- and nanoscale with high precision. Here, we review the physical and chemical mechanisms of microscale surgery, and the corresponding design principles, applications, and implementations in microfluidic systems. We consider four types of surgical operations: 1) Sectioning, which splits a biological entity into multiple parts, 2) ablation, which destroys part of an entity, 3) biopsy, which extracts materials from within a living cell, and 4) fusion, which joins multiple entities into one. For each type of surgery, we summarize the motivating applications and the microfluidic devices developed. Throughout the review, we highlight existing challenges and opportunities. We hope that this review will inspire scientists and engineers to continue to explore and improve microfluidic surgical methods.
\end{abstract}

Keywords: Microfluidics; surgery; single cell; multicellular systems; sectioning; ablation; biopsy; fusion

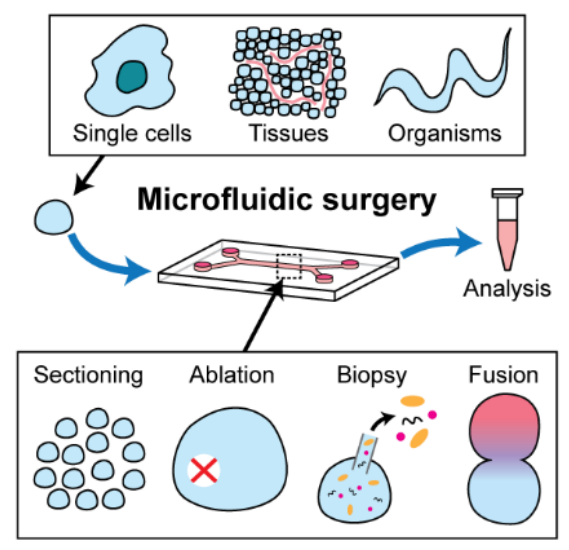

\section{CONTENTS}

\section{Introduction}

1.1 Brief history of surgery on microscopic organisms and single cells

1.2 Microfluidics for surgical operations on single cells and small multicellular systems

1.3 Scope and organization of the review

2. Principles of microscale surgery and implications for the design of microfluidic systems

2.1 Methods to create a cut or opening

2.1.1 Mechanical methods

2.1.1.1 Cutting single cells

2.1.1.2 Cutting multicellular systems

2.1.1.3 Implications for the design of microfluidic surgery
2.1.2 Optical methods

2.1.2.1 Implications for the design of microfluidic surgery

2.1.3 Chemical methods

\subsubsection{Surfactants}

2.1.3.2 Enzymes

2.1.3.3 Implications for the design of microfluidic surgery

2.1.4 Electrical methods

2.1.4.1 Implications for the design of microfluidic surgery

2.2 Methods to fuse

2.2.1 Virus-mediated fusion

2.2.2 Polyethylene glycol (PEG)-mediated fusion

2.2.3 Electrofusion 
2.2.4 Implications for the design of microfluidic surgery

2.3 Methods to transport

2.3.1 Pressure-driven flow

2.3.2 Electrokinetic flow

2.3.2.1 Electroosmosis

2.3.2.2 Electrophoresis

2.3.2.3 Dielectrophoresis

2.3.3 Electrowetting

2.3.4 Diffusion-mediated transport

2.3.5 Implications for the design of microfluidic surgery

\section{Sectioning}

3.1 Single cells

3.1.1 Enucleation

3.1.2 Bisection

3.1.3 Fragmentation

3.2 Cell division

3.3 Multicellular systems

3.3.1 Dissociation into single cells

3.3.2 Sectioning into small tissue fragments

3.3.3 Bisection

3.4 Outlook and future opportunities

\section{Ablation}

4.1 Single cells and subcellular structures

4.1.1 Axotomy

4.1.1.1 Axotomy by vacuum aspiration

4.1.1.2 Axotomy by introduction of surfactants

4.1.1.3 Axotomy by compression

4.1.1.4 Axotomy by laser ablation

4.1.2 Microtubule lattice damage

4.2 In vitro cultured cells

4.2.1 Wounding by compression

4.2.2 Wounding by introduction of chemicals

4.2.3 Wounding by application of hydrodynamic shear force

\section{INTRODUCTION}

\subsection{Brief history of surgery on microscopic organisms and single cells}

Surgical operations on small organisms have been performed as early as the 1600 s to make fundamental and often revolutionary discoveries on anatomical structures and biological processes. Marcello Malpighi (1628-94), an Italian anatomist, was credited as the first naturalist to attempt microdissection. ${ }^{1}$ Malpighi combined microscopy and careful dissecting skills for the microdissection of a mulberry silk moth, which was only $13 \mathrm{~mm}$ long. Remarkable anatomical details of the internal organs of the insect were reported. It was believed that Malpighi used a single-lens microscope, but its optics were poor. Furthermore, Malpighi had to invent instru-
4.3 Multicellular organisms

4.3.1 Immobilization by applying pressure

4.3.2 Immobilization by cooling

4.3.3 Passive immobilization

4.4 Outlook and future opportunities

\section{Biopsy}

5.1 Transport driven by electric field

5.1.1 Nanopipettes

5.1.2 Dielectrophoretic nanotweezer

5.2 Diffusion following electroporation

5.2.1 Nanostraws

5.2.2 Nanoporous membrane

5.3 Pressure-driven flow

5.3.1 Fluidic force microscopy

5.3.2 Extended nanochannel

5.4 Outlook and future opportunities

\section{Fusion}

6.1 Single cells

6.1.1 Polyethylene glycol (PEG)-mediated fusion

6.1.2 Electrofusion

6.1.2.1 Microorifice

6.1.2.2 Microelectrode arrays

6.1.2.3 Droplet encapsulation

6.1.2.4 Cell traps

6.1.3 Virus-mediated fusion

6.2 Multicellular systems

6.3 Outlook and future opportunities

\section{Summary and perspectives}

7.1 Summary

7.2 Perspectives

Author Information

Acknowledgements

References

ments for microdissection since they did not exist yet. Unfortunately, no details of the tools were described. ${ }^{2}$ Regardless, the experiments on such a small scale were incredibly difficult and required extreme dexterity. In fact, Malpighi wrote that "My dissertation on Bombyx was extremely tiring and laborious, because of the novelty, minuteness, fragility and entanglement of the parts. Carrying out the task therefore made it necessary to develop entirely new methods. And since I pursued this exacting work for many months without respite, in the following autumn I was afflicted with fevers and an inflammation of the eyes". 3

It was not until the late 1800 s that mechanical micromanipulators were beginning to be constructed to hold microneedles, to be used along with improved microscopy for microdissection, primarily on single cells. ${ }^{4,5}$ 


\section{“Micrurgy" (Barber's method), 1900s}
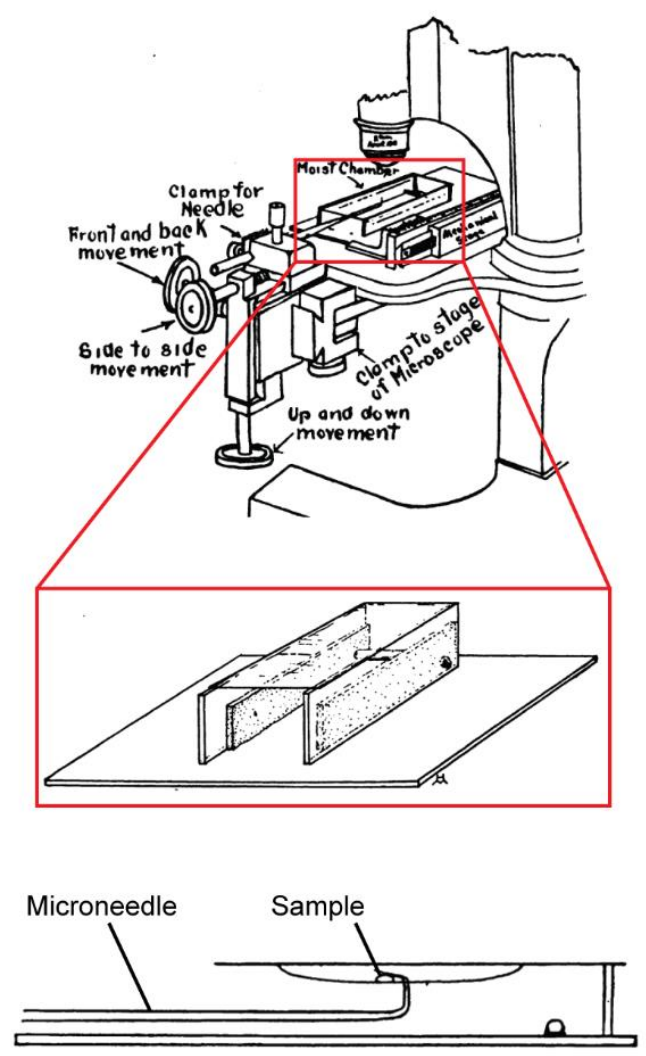

Microfluidic surgery, 2000s
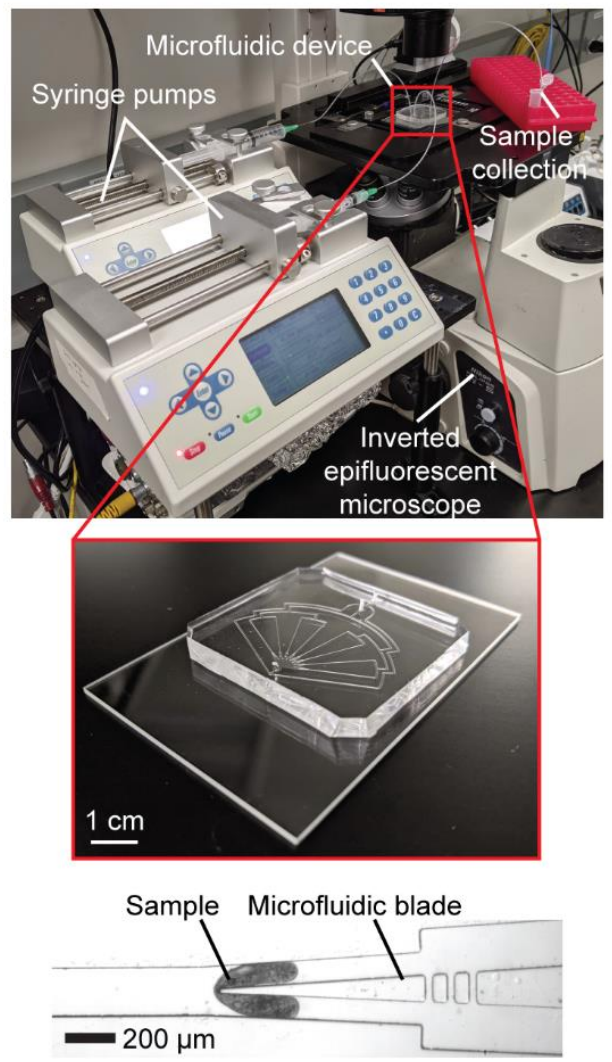

Figure 1. Comparison of "micrurgy" vs. microfluidic surgery systems. Full system (top), microenvironment (inset), and surgical tool (bottom) shown for each. In "micrurgy" as described by Barber (left), samples are contained in a moist glass chamber. The surgical operation and sample positioning are controlled by hand via mechanical screw assemblies. The microtool is a pulled microneedle, and the imaging is brightfield microscopy. Adapted from Chambers. Biol. Bull., 1918, 34, 121. ${ }^{5}$ In microfluidic surgery (right), using the microfluidic guillotine as an example, samples are contained in a microfluidic channel. The surgical operation is controlled automatically via syringe pumps and channel geometry. The microtool is a microblade fabricated inside the channel. Eight parallel channels and microblades allow parallel surgery with many organisms simultaneously (see details in Section 3). Multiple imaging modalities including fluorescence microscopy may be used. ${ }^{6,7}$

Also referred to as micrurgy, the most comprehensive method was perhaps the one described by Marshall Barber in the early 1900s (Figure 1). ${ }^{8-12}$ Barber's setup consisted of a moist chamber where a hanging drop containing living cells was suspended at the roof of a glass box. A dissecting microneedle, held by a needle holder which was clamped on a microscope stage, could be moved in 3 directions using screws. The microneedle was made by pulling a glass tubing over a flame and bending it at right angles $2-3 \mathrm{~mm}$ from the pointed tip. The needle was inserted into the moist chamber with its tip pointing up into the hanging drop, pressing the cell against the roof of the glass chamber. As such, the microneedle did not obstruct the view of the cell from the microscope objective. The entire setup was mounted under a microscope, thereby allowing dissection and observation under the highest magnification then possible. Modifications by Robert Chambers to the mechanical design, referred to as the "microvivisection method", enhanced the control and movement of the microtool, which was subsequently commercialized by the Leitz company. 5,13
While these methods have improved the precision of microscale surgery and have allowed the investigation of subcellular structures (e.g. chromosomes) and biological processes (e.g. fertilization) in single cells, they remained labor and time intensive, required fine motor skills, and were limited to the manipulation of one cell or organism at a time. Nevertheless, these methods laid the foundation for microscale surgery by describing its essential elements (Table 1): 1) a moist chamber to provide a suitable microenvironment for the living cells or organisms, 2) a micromanipulator to control the location of surgery, 3) a microtool to perform the dissection, and 4) microscopy to guide the positioning of the microtool over the sample optically, and to observe the sample after surgery.

1.2 Microfluidics for surgical operations on single cells and small multicellular systems

Microfluidics, the science and technology of processing small amounts of fluids in channels with dimensions less than hundreds of micrometers, has allowed unprecedented capability to manipulate objects, living or nonliving, in the micro and 
Table 1. Comparison of the elements of microscale surgery ("micrurgy") described by Marshall Barber in the early 1900s vs. microfluidic surgery, where most publications started in the 2000 s.

\begin{tabular}{|l|l|l|}
\hline & "Micrurgy" (early 1900s) & Microfluidic surgery (2000s) \\
\hline Microenvironment & $\begin{array}{l}\text { Hanging drop in moist } \\
\text { chamber made of glass }\end{array}$ & $\begin{array}{l}\text { Enclosed microfluidic channel made of poly(dimethylsiloxane) } \\
\text { (PDMS)/glass; precise control of temperature, gas composition, pH bio- } \\
\text { chemical composition of the media }\end{array}$ \\
\hline $\begin{array}{l}\text { Positioning of the } \\
\text { surgical tool to the } \\
\text { sample }\end{array}$ & $\begin{array}{l}\text { Mechanical micromanipulator } \\
\text { operated by screws }\end{array}$ & $\begin{array}{l}\text { Confinement in a narrow channel; laminar flow; pneumatic valves; hydro- } \\
\text { dynamic traps; dielectrophoretic trapping; piezoelectric translation stage }\end{array}$ \\
\hline Surgical tool & $\begin{array}{l}\text { Microneedles or micropipettes } \\
\text { made by pulling a glass pipette }\end{array}$ & $\begin{array}{l}\text { Sharp micro-knives fabricated inside the microchannel; nanopipettes; hy- } \\
\text { drodynamic shear and extension; laser ablation; biochemical agents; elec- } \\
\text { tric field }\end{array}$ \\
\hline Optics & Simple brightfield microscopy & $\begin{array}{l}\text { Brightfield; epifluorescence; confocal fluorescence microscopy; high speed } \\
\text { imaging }\end{array}$ \\
\hline
\end{tabular}

nanoscale. ${ }^{14}$ The application of microfluidics to biology has demonstrated successful operations including the culture, treatment, selection, lysis, separation, and analysis of single cells and multicellular systems (organoids, tissues, and organisms). ${ }^{15}$ The characteristics and advantages of microfluidics have been described in many prior excellent reviews. ${ }^{16-20}$ The features that are of particular relevance to microscale surgery are: 1) precise control of the microenvironment in an enclosed system, 2) immobilization and precise positioning of the sample relative to the surgical tool while performing the surgery, 3 ) ease of integration with mechanical, electrical, optical and other components for trapping, imaging, and/or performing the surgery, and 4) continuous flow to flush the sample in and out of the surgical area, thereby achieving a high throughput of surgery. Figure 1 and Table 1 show the components of microfluidic surgery compared with those in Barber's method.

With technical advances enabled by microfluidics, a broad range of applications is now possible. The applications of microfluidic surgery fall into two main categories. The first category is to understand how biological systems work. For example, ablation surgery has been performed to probe the biophysical properties of cellular structures, and the mechanism of wound healing and regeneration. The second category is to engineer cells or multicellular systems for biomedical needs. Examples include enucleation surgery for cell reprogramming and fusion surgery to create hybridomas for the production of monoclonal antibodies.

\subsection{Scope and organization of the review}

The objective of this review is to summarize the principles of microscale surgery, describe the design of microfluidic systems as guided by these principles, and review microfluidic devices and related applications that have been developed to perform surgery on cells or small multicellular systems less

than a few millimeters in size. The review is intended for anyone interested in microfluidic surgery, including biologists and cellular engineers looking for the best method for their study, physicists or chemists looking to further the understanding of the interactions between biological tissues and physical or chemical forces, and engineers looking to develop new technologies to improve the current surgical methods.

In this review, we consider an operation as surgery if parts of the sample are physically opened, destroyed, or removed for further analysis, and if the sample remains viable after the operation. As such, all operations involving the lysis of cells are excluded. Although previous reviews exist on subcategories of microfluidic surgery, none, to our knowledge, are as comprehensive, and few discuss the principles of surgery and implications for microfluidic design.

We classify surgical operations into the following categories: 1) Sectioning, where a biological entity (a single cell or multicellular system like a tissue or an organoid) is separated into multiple distinct parts. 2) Ablation, where parts of the biological entity are removed or destroyed. 3) Biopsy, where materials are extracted from within a living cell. 4) Fusion, also referred to as grafting in some literature, where multiple biological units are joined to form a single entity (Figure 2). We have excluded the injection of materials into a cell since this topic has been covered by a recent comprehensive review. $^{21}$

The rest of the review is organized as follows. Section 2 discusses the principles of microscale surgery, i.e., the mechanisms underlying methods to introduce cuts, to fuse, and to transport materials from a biological sample. Directly after each principle, we discuss the implications and requirements for the design of microfluidic systems utilizing the respective principle. Sections 3 to 6 summarize microfluidic systems for the four types of surgery mentioned above: sectioning, ablation, biopsy, and fusion. At the end of each of these sections, we include a short summary of existing challenges and opportunities. Section 7 concludes the review with a discussion of future outlook. 


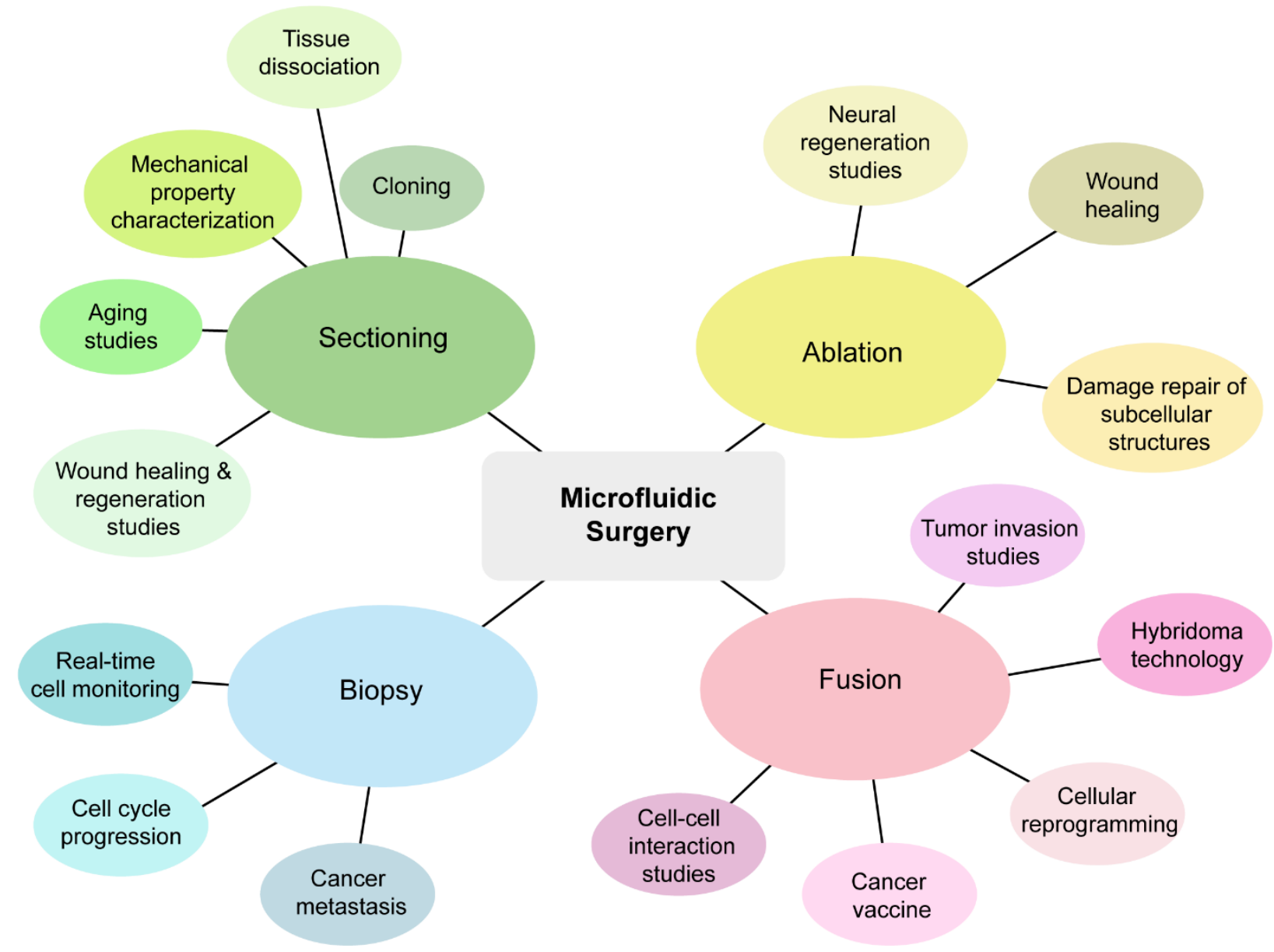

Figure 2. Overview of microfluidic surgery. The four categories of microfluidic surgery and selected applications are shown.

\section{PRINCIPLES OF MICROSCALE SURGERY AND IMPLICATIONS FOR THE DESIGN OF MICROFLU- IDIC SYSTEMS}

All surgery involves the application of external forces to disrupt parts of a cell, tissue, or organism. In this section, we summarize the types of forces that have been applied in microfluidic surgery, and discuss their working principles. We organize this section by the methods to create a cut or opening, to fuse, and to transport materials from the cell, tissue, or organism. Since extensive literature exists on each of the principles, we will only provide an overview here and refer the readers to the cited references for further information. Based on the principles, we will also discuss the implications and requirements for the design of microfluidic systems to perform the surgery.

\subsection{Methods to create a cut or opening}

\subsubsection{Mechanical methods}

To mechanically cut a biological material, a sufficiently large mechanical load must be applied to rupture the material. This mechanical load is typically applied using a sharp solid object, e.g., a knife blade or a needle.

\subsubsection{Cutting single cells}

At the single cell level, the mechanical cutting process involves rupturing the plasma membrane and the underlying cytoskeleton. The failure of a lipid bilayer can be described by the activation energy theory. ${ }^{22}$ In this theory, thermally activated molecular-scale defects open and close spontaneously in membranes. The membrane ruptures when the defect crosses a cavitation barrier and becomes an unstable hole. Increased normal or tensile stress on the membrane can lower the activation energy of both defect formation and the cavitation barrier, which increases the frequency of defect and unstable hole formation, and thus the frequency of rupture.

The role of a sharp object is to introduce a local increase in normal stress or membrane tension past a critical value to

cause rupture (Figure 3A). The critical tension for rupture has been studied in lipid vesicles and live cells, and is found to be $\sim 1-10 \mathrm{mN} / \mathrm{m}$, increasing with strain rate. ${ }^{22,23}$ Although higher strain rates require higher critical tensions for rupture, the typical time required to reach the critical tension decreases faster than the critical tension increases. The frequency of defect and hole formation increases with increasing tension. ${ }^{22}$ Thus, it is often easier and more convenient to rupture membranes at high strain rates.

For objects with a tip radius $>20 \mathrm{~nm}$, the contribution by normal stress is expected to be negligible compared with the increase in membrane tension. ${ }^{23}$ The total tension $\mathrm{T}_{0}$ in the cell 

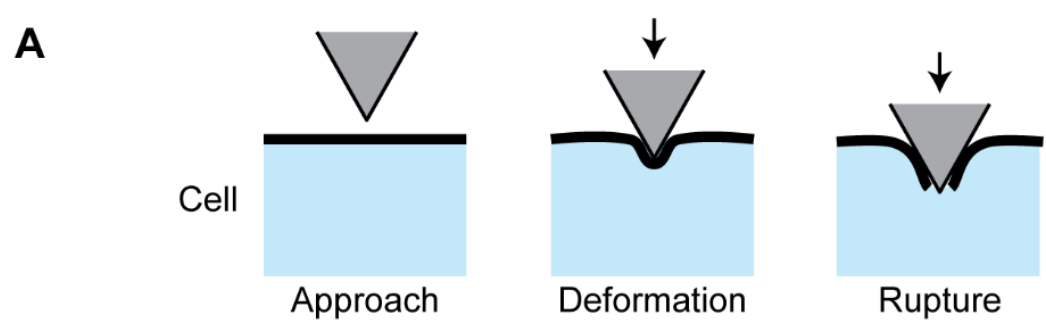

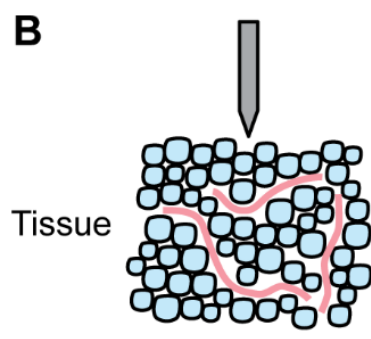

Approach

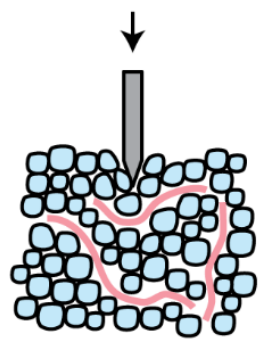

Deformation

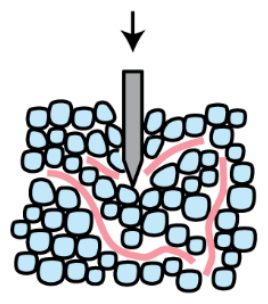

Fracture

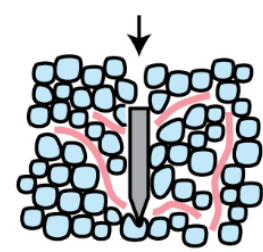

Crack propagation

Figure 3. Stages of mechanically creating a cut or opening in a biological sample. A sharp solid tool is used to cut (A) a single cell or (B) tissue. As the tool contacts and then deforms the sample, the mechanical load on the sample increases and the sample is cut at a critical load.

membrane during indentation and cutting can be expressed as (Eq. 1),

$$
T_{0}=T_{p}+T_{b}+T_{a} \quad \text { (Eq. 1) }
$$

where $T_{p}$ is the intrinsic tension $(\sim 1 \mathrm{mN} / \mathrm{m}), T_{b}$ is the bending tension, and $T_{a}$ is the indentation tension arising from area deformation. $^{23,24}$ The bending tension is given by $T_{b}=$ $\frac{1}{2} K_{b}\left(\frac{2}{R}\right)^{2}$, where $K_{b}$ is the bending modulus of the membrane and $R$ is the tip radius of the indentation tool. The bending modulus can be found using $K_{b}=\frac{E b^{3}}{12}$, where $E$ is the Young's modulus of the membrane and $b$ is the lipid bilayer thickness $(\sim 6 \mathrm{~nm})$. The indentation tension is given by $T_{a}=K_{a} \alpha$, where $K_{a}$ is the expansion modulus given by $K_{a}=E b$ and $\alpha$ is the fractional area dilation. In this description, $T_{p}$ and $T_{b}$ can be assumed as constant after the tool tip has made full contact with the membrane. Further indentation past this point only increases $\alpha$ and $T_{a}$ until the critical tension is reached. For red blood cells, the critical area dilation for membrane rupture has been found to be $2-4 \%$. $^{24-26}$

From the theory outlined above, the Young's modulus or the stiffness of the membrane determines how much indentation is needed before rupture occurs. Soft cell membranes in general require larger indentation depths to rupture compared with stiff cell membranes at the same loading rate. The observed Young's modulus of cell membranes vary widely, from $\sim 1$ - 2 MPa for neuronal cells, ${ }^{27} \sim 10-30 \mathrm{MPa}$ for lymphocytes, ${ }^{28}$ to $\sim 50$ - $75 \mathrm{MPa}$ for red blood cells. ${ }^{24,25}$ Here, the plasma membrane and the underlying cytoskeleton is simplified as an isotropic elastic sheet, with a single set of mechanical parameters. ${ }^{25}$ However, recent molecular simulations of the plasma membrane have suggested the relationship between $E, K_{b}$, and $K_{a}$ may be more complex than previously thought. ${ }^{29}$

Instead of defining a critical tension, an alternative way to characterize membrane rupture is by measuring the applied mechanical load. Using an AFM indenter with a tip diameter of $2-10 \mu \mathrm{m}$, the critical stress for cell membrane rupture in bovine aortic endothelial cells was found to be approximately $12 \mathrm{kPa}^{30}$

In summary, while the detailed model of membrane rupture is still an area of active research, a key insight that informs the design of cutting devices is that sharp tips tend to increase membrane bending, stretching, and local tension, thereby reducing the force required to initiate membrane rupture. ${ }^{31}$

\subsubsection{Cutting multicellular systems}

At the multicellular level, the mechanical cutting process involves fracturing both the cells and the extracellular matrix. At this scale, the cutting process can be described using fracture mechanics. ${ }^{32}$ The biological material can be modelled as

linear elastic, viscoelastic, or hyperelastic. For most studies, the cells and the extracellular matrix are combined and described by a single set of bulk mechanical properties.

The fracture mechanics description includes three main stages (Figure 3B). First, the material deforms under an applied load. Second, the material ruptures when its fracture stress is reached and a crack is initiated. Third, the cutting becomes steady and the crack propagates. This approach has been used to describe the cutting of a bulk tissue, ${ }^{33}$ the cutting of a thin biological membrane (e.g. skin), ${ }^{34}$ and the insertion of a needle into a tissue. ${ }^{35}$

The toughness of biological tissues varies by tissue type, fracture failure mode, and crack geometry. The fracture failure modes involved in cutting biological tissues are typically mode I (opening, e.g. needle insertion or cutting with a single blade) and mode III (out-of-plane shear, e.g. cutting with scissors or tearing). For example, the toughness in mode I of liver $\left(\sim 160 \mathrm{~J} / \mathrm{m}^{2}\right)^{36}$ is much lower than that of aorta tissue $(\sim 1$ $\left.\mathrm{kJ} / \mathrm{m}^{2}\right){ }^{37}$ and skin $\left(\sim 30 \mathrm{~kJ} / \mathrm{m}^{2}\right.$ in mode I and $\sim 20 \mathrm{~kJ} / \mathrm{m}^{2}$ in mode III). ${ }^{38}$ 
Mode I fracture is the most relevant to the microfluidic surgical methods described in this review. The mode I fracture process can be described using an energy balance formulation by (Eq. 2).

$$
F d u+d U_{i}=J_{I C} d A+d \Delta+d \Gamma+P d u \quad \text { (Eq. 2) }
$$

where $F$ is the cutting force, $d u$ is the incremental displacement of the cutting tool, $U_{i}$ is the internal strain energy stored in the sample prior to cutting, $J_{I C}$ is the mode I toughness of the material (also referred to as the strain energy release rate), $d A$ is the incremental increase in crack area, $\Delta$ is the elastic strain energy, $\Gamma$ is the inelastic strain energy, and $P$ is the friction force..$^{32,34,35,39}$ For linear elastic fracture, without friction, the strain energy release rate (i.e. $J_{I C}$ ) scales $\sim E r_{c}$ where $E$ is the Young's modulus of the material and $r_{c}$ is the radius of the crack tip. ${ }^{40,41}$ The crack tip radius $r_{c}$ is not necessarily equal to the tool tip radius $r_{t}$, but the fracture energy may still scale with $r_{t}$. A study using razor blades to cut PDMS samples found that the strain energy release rate scaled as a power law $\sim E r_{t}^{n}$, where $n$ ranged from $\sim 0.8-1.4$ for samples with Young's modulus $\sim 0.9 \mathrm{MPa}-0.3 \mathrm{MPa}$, respectively. ${ }^{42}$ This result suggests that smaller tool tip radii generate smaller crack tip radii and result in a lower energy required to fracture, as posited previously by Lake and Yeoh. ${ }^{40}$ Furthermore, it appears that the scaling relationship between $r_{t}$ and $J_{I C}$ is similar to that between $r_{c}$ and $J_{I C}$. However, the precise relationship between fracture energy, crack geometry, and tool geometry is complex and remains an open question, especially for nonlinear biological materials.

When cutting tissues, a lower cutting force is desirable as it typically leads to smaller tissue deformation, yields a higher quality cut, and reduces unnecessary damage to both the blade and the tissue.$^{33,43}$ For typical conditions when using a surgical blade or needle, the required force is on the order of $1-10 \mathrm{~N}$ for liver (toughness $\left.\sim 160 \mathrm{~J} / \mathrm{m}^{2}\right)^{36}$, aorta $\left(\sim 1 \mathrm{~kJ} / \mathrm{m}^{2}\right),{ }^{44}$ and elastomer surrogates $\left(\sim 2-4 \mathrm{~kJ} / \mathrm{m}^{2}\right){ }^{43,45}$ From the theory above, sharper cutting tools reduce $J_{I C}$ and thus reduce the cutting force $F$. Experiments on using surgical blades have found that smaller blade wedge angles and tip radii (i.e., sharper blades), moderate blade inclination angles, and faster cutting speeds contribute to lower force and tool displacement required to initiate a fracture and cut the tissue..$^{33,43-45}$

\subsubsection{Implications for the design of microfluidic sur-} gery

The cutting principles for single cells and tissues described above can inform the experimental design of microfluidic cutting methods. The cutting edge should be as sharp as possible to increase the stress at the edge and reduce the required cutting force, which yields higher quality, cleaner cuts. ${ }^{43,45}$ Conventional surgical blades have blade tip widths on the order of $100 \mathrm{~nm} .{ }^{46}$ This degree of sharpness can be fabricated using standard micro or nanofabrication techniques, soft lithography, ${ }^{47}$ or high-resolution 3D printing. ${ }^{48,49}$

Another consideration is the stiffness of the cutting tool. Stiffer materials are preferred, as they deform and break less easily and cut more efficiently. ${ }^{33}$ Poly(dimethylsiloxane) (PDMS) has a stiffness of $\sim 2 \mathrm{MPa},{ }^{50,51}$ far less than that of steel scalpel blades ( 180-200 GPa) and some tissues. ${ }^{52} \mathrm{Nev}-$ ertheless, PDMS blades have been successful in cutting very soft cells and organoids (see Section 3). Some studies have explored using stiff polymers or silicon in microfluidic devices to overcome these limitations. ${ }^{53,54}$ However, factors such as the ease of fabrication and integration with the rest of the microfluidic system must be considered when selecting a material for the cutting tool.

Ultimately, the success of mechanical cutting in a microfluidic device depends on an interplay between the geometry, tool and sample material, and cutting parameters. Certain parameters such as blade inclination angle can be more difficult to change during an experiment in a microfluidic device. An alternative is to compensate by adjusting other parameters such as the flow profile and flow rate to achieve sufficiently high forces and stresses for cutting the sample.

\section{Photochemical damage}

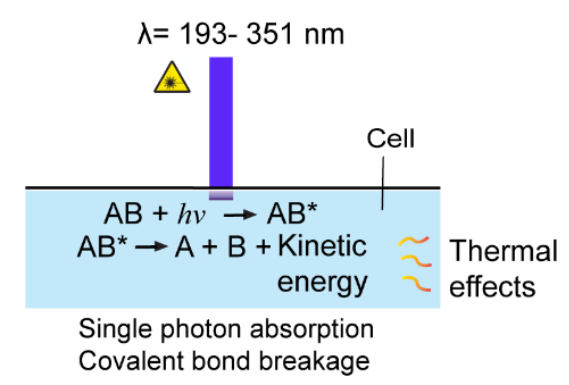

Intensity threshold $0.26 * 10^{12} \mathrm{~W} / \mathrm{cm}^{2}$

Pulse repetition rate $>1 \mathrm{MHz}$
Plasma-mediated ablation

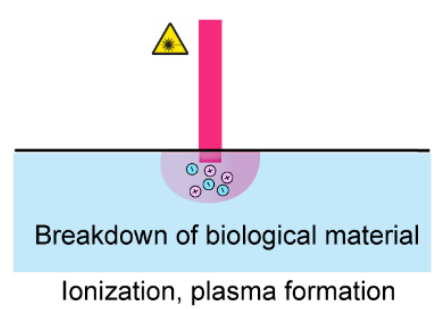

Intensity threshold $6.54 * 10^{12} \mathrm{~W} / \mathrm{cm}^{2}$ Pulse repetition rate $<1 \mathrm{MHz}$
Thermoelastic stress confinement

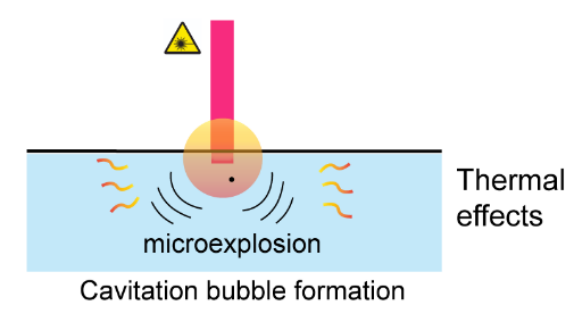

Intensity threshold $5.1 * 10^{12} \mathrm{~W} / \mathrm{cm}^{2}$

Pulse repetition rate $<1 \mathrm{MHz}$

Figure 4. Types of effects of laser light on biological samples. Laser light ablates biological samples by either photochemical damage, plasma-mediated ablation or thermoelastic stress confinement. Photochemical damage only takes place under UV-light. Intensity threshold, a measure of the spatial concentration of the power and pulse repetition rate are indicated. ${ }^{55}$ 


\subsubsection{Optical methods}

Laser ablation is the primary method of cutting biological structures optically. It involves focusing a high energy laser onto a specific location within a cell or tissue to ablate a desired structure. Compared with mechanical methods of cutting, lasers can ablate structures with a much higher spatial resolution. Importantly, internal structures can be focused and ablated without cutting open the cell, tissue, or organism, as long as the biological material is transparent to the laser light.

The interaction between laser light and biological materials is complex. ${ }^{56}$ When biological tissues are exposed to laser light, at least three types of effects have been identified (Figure 4). ${ }^{55}$

(i) Photochemical damage occurs when a single photon is absorbed to result in the excitation of electrons and dissociation of molecules. However, this regime is highly wavelength dependent and occurs at UV wavelengths $(193 \mathrm{~nm}-351$ $\mathrm{nm}) .{ }^{56-58}$

(ii) Plasma-mediated ablation occurs when multiple photons are absorbed by the tissue, causing electrons to enter excited states, which further cause ionization and the formation of electron-ion plasma. Electrons may excite other unexcited electrons in a process known as avalanche ionization. Ionization can alter the nature of biological material and induce its breakdown, and can also lead to photochemical damage. ${ }^{59}$

(iii) Thermoelastic stress confinement is caused by the high temperature and high pressure plasma where the thermal stresses overcome the tensile strength of the medium around the plasma, thus causing a micro-explosion and cavitation bubbles. Local temperature rise, boiling and cavitation bubbles can cause mechanical disruption of structures. ${ }^{59,60}$

Many microfluidic laser ablation surgery studies have used pulsed lasers in the near IR wavelengths $(\sim 800 \mathrm{~nm})$, pulse durations from $100 \mathrm{fs}-3 \mathrm{~ns}$, repetition rates of $1 \mathrm{kHz}-80$ $\mathrm{MHz}$, and pulse energies from $5-20 \mathrm{~nJ}{ }^{61-66}$ Due to the very short pulses (fs to ps), even low pulse energies can achieve high enough peak power for ablation. ${ }^{59}$ The damage is also localized to extremely small regions. A sub-femtoliter volume of the cell or tissue can be ablated without affecting adjacent areas. ${ }^{67}$

The parameters that determine the effects of laser ablation on biological tissues include the laser wavelength, repetition rate, pulse duration, pulse intensity, and the number of pulses. ${ }^{55}$ Although some studies have used UV wavelengths (340 $\mathrm{nm}-440 \mathrm{~nm}$ ) for ablation, ${ }^{62,68,69} \mathrm{UV}$ lasers can cause photodamage to cells and subsequent cell death by the formation of reactive oxygen species. In a study on the viability of cells 12 hours post-laser ablation, it was found that cells ablated with a $337 \mathrm{~nm}$ laser (3 ns pulses) had lower viability than cells ablated with a $770 \mathrm{~nm}$ two-photon laser (110 fs pulses).$^{58}$

Both low $(1-250 \mathrm{kHz})$ and high $(\sim 80 \mathrm{MHz})$ repetition rates have been used for femtosecond laser surgery. Ablations at low repetition rates use larger pulse energies and tend to cause the formation of transient cavitation bubbles due to thermoelastic perturbation. Ablations at higher repetition rates use lower pulse energies and rely primarily on the photochemical damage of biological structures. ${ }^{70,71}$ In one study investigating the cellular response to irradiation at $532 \mathrm{~nm}$ with pulse duration varying from $180-1100 \mathrm{ps}$, shorter pulse durations were found to reduce the threshold laser pulse energy for plasma formation and cavitation bubble energy, and correlate with better spatial precision of laser ablation. ${ }^{72}$

\subsubsection{Implications for the design of microfluidic sur- gery}

To be compatible with laser ablation, the microfluidic channels must be transparent to the laser. Both PDMS and glass are transparent from the UV to near IR wavelengths. ${ }^{73}$ Along with their chemical inertness, devices made of PDMS and glass are compatible with laser surgery, as well as post-surgery viability monitoring. ${ }^{69}$

Despite its high precision, a major drawback of laser ablation is its low throughput. Integration with a microfluidic flow-through system can increase the throughput dramatically. For example, a sample can be introduced into the focal point of the laser, become ablated, and then be flushed out of the system immediately in a continuous manner.

Prior to laser ablation, the positioning and immobilization of the sample being ablated is critical. Laser surgery in single cells and small organisms is thus assisted significantly by the use of microfluidics to provide the confinement and positioning of cells or organisms (see details in Section 4).

\subsubsection{Chemical methods}

\subsubsection{Surfactants}

Surfactants, or detergents, are amphiphilic molecules with a polar head group and a hydrophobic tail. They can solubilize hydrophobic or lipid molecules and destabilize lipid membranes. ${ }^{74,75}$ Experimental evidence of membrane disruption has been found as early as 1962, when electron micrographs of membranes of the Rous sarcoma virus as well as erythrocytes showed holes with size $\sim 80 \AA$ when treated with a surfactant consisting of a saponin solution. ${ }^{76}$

A common feature between phospholipids, the constituent molecules of the lipid bilayer, and surfactants is that they are both amphiphilic. However, the shape of the molecules, and therefore their packing and their preferred curvature, are different. Phospholipids are typically idealized as a cylinder (with a packing parameter $\mathrm{p} \sim 1$ ). They favor the assembly of flat bilayers with a spontaneous curvature that is close to zero or slightly negative. Surfactants such as Triton are idealized as a cone $(\mathrm{p}<1)$. Thus, these molecules favor the formation of curved assemblies or micelles with a positive spontaneous curvature. ${ }^{77,78}$

In terms of the mechanism for lipid bilayer destabilization, the surfactant inserts itself into the membrane asymmetrically (Figure 5A) (for example, in the outer membrane monolayer but not in the inner membrane monolayer). ${ }^{75}$ Thus, lipids in the layer incorporating surfactant molecules may be compressed, or the underpopulated layer may expand, to rectify this asymmetric insertion. This causes curvature strain, that is, the bilayer cannot curve in a normal fashion. Curvature strain can also be experienced within each monolayer of a bilayer. The strain can lead to several outcomes. The membrane might become more flexible, as the lipids composing the membrane become more disordered. The membrane may transiently fail. Curvature strain can be reduced by the reorganization of surfactant molecules into toroidal structures that form the edges of a pore in the membrane to account for the presence of the surfactant. ${ }^{75,79}$ Some surfactants (e.g., saponin) may form 
A

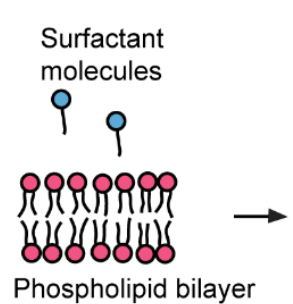

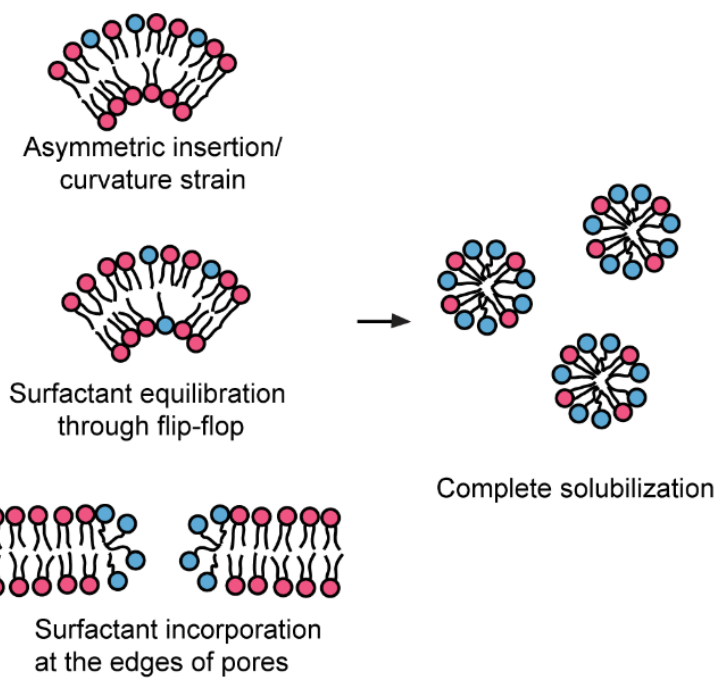

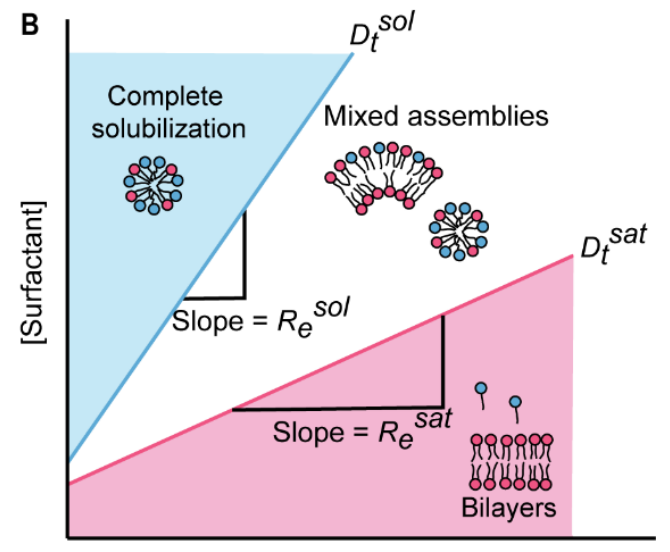

[Lipid]

Surfactant incorporation

Figure 5. Action of surfactants on lipid bilayers. (A) Mechanism of action is at the molecular level. (B) Phase diagram of lipidsurfactant-water system. At low surfactant concentrations, assemblies exist in bilayer configuration. With increasing surfactant, mixed assemblies are formed and finally micelles are generated on complete solubilization. $D_{t}^{s a t}$ and $D_{t}^{s o l}$ are surfactant concentrations at the start and end of solubilization and $R_{e}^{\text {sat }}$ and $R_{e}{ }^{\text {sol }}$ are the molar ratio of surfactant to lipid in an assembly at the onset and the end of solubilization, respectively. ${ }^{78}$

complexes with molecules such as cholesterol that are found in biological membranes. As cholesterol can stabilize membranes mechanically, the removal of cholesterol can disrupt these membranes. ${ }^{75,80-82}$

The generally accepted model of the progression of membrane solubilization involves 3 stages. ${ }^{83}$ In stage 1 ("detergent binding"), the surfactants partition into the lipid bilayer. In stage 2 ("lamellar-micellar phase transition"), the membrane is saturated with surfactant and lipid-saturated micelles are in coexistence. Here, the proportions between lamellae and micelles change but their internal compositions (i.e., the surfactant-to-lipid ratios) are constant. In stage 3 (complete solubilization), no lamellar structures are left and only micelles remain.

The different stages can be represented as a phase diagram plotted between surfactant and lipid concentration (Figure 5B). ${ }^{77}$ The critical surfactant concentrations, $D_{t}^{\text {sat }}$ and $D_{t}^{\text {sol }}$, denote the surfactant concentrations needed for the onset and the end of solubilization of the lipid membrane, respectively. The slopes of the two lines, $R_{e}{ }^{\text {sat }}$ and $R_{e}{ }^{\text {sol }}$, are the molar ratio of surfactant to lipid in an assembly at the onset and the end of solubilization, respectively.

Physically, these values represent how much surfactant is needed to solubilize phospholipid bilayers. Strong surfactants with polar head groups and alkyl tails have typical $R_{e}^{\text {sat }} \sim 0.5$. The solubilization of 1-palmitoyl-2-oleoyl-sn-glycero-3phosphocholine (POPC) by Triton X-100 starts at $R_{e}^{\text {sat }} \sim 0.4$ (i.e., a molar ratio of 0.4 surfactants to POPC) ${ }^{84}$ Weak surfactants $\left(R_{e}^{\text {sat }}>1\right)$ initiate solubilization only when more than half of the molecules in the membrane are surfactants. ${ }^{75}$ Furthermore, it has been found that $R_{e}{ }^{\text {sat }}$ scales with the ratio of the spontaneous curvature of the lipid to that of the surfactant. Solubilization is thus enhanced by using a surfactant with a higher positive spontaneous curvature, or when the lipid has a less negative spontaneous curvature. For example, the solubilization of 1,2-Dioleoyl-sn-glycero-3-phosphoethanolamine (DOPE), which has a strong negative spontaneous curvature, requires high concentrations of surfactant $\left(R_{e}^{s a t} \sim 2\right.$ using Triton-X100). ${ }^{75}$

Due to their action on lipids, surfactants have found use in single cell surgery involving the disruption of the plasma membrane. Examples of surfactants used in studies of plasma membrane disruption include saponin, Triton X-100 and sodium dodecyl sulfate (SDS), typically at $0.1 \%$.

\subsubsection{Enzymes}

At the multicellular level, the most common chemical method to remove cells is by using the enzyme trypsin for disrupting the adhesion between cells. Trypsin is a proteolytic enzyme that uses the amino acid serine in its active site. It is produced by the pancreas and cleaved to its final form in the small intestine. It was named by Kuhne in 1876 who described the proteolytic activity of this enzyme. ${ }^{85,86}$ Trypsin was crystallized in 1931 by Northrop and Kunitz. ${ }^{87}$

Trypsin cleaves proteins at the C-terminal of lysines and arginines, a process known as trypsinization. ${ }^{88}$ When applied to adherent cells or tissues, trypsin digests the protein matrix that binds cells in the tissue as well as other cell surface proteins such as cadherins and integrins. This digestion causes the detachment of cells from culture substrates and subsequent rounding of the detached cells (Figure 6) ${ }^{89,90}$ Trypsin is generally inactivated by the addition of serum, which contains trypsin inhibitors that are components of the $a_{1^{-}}$and $a_{2^{-}}$ globulin..$^{91,92}$ 

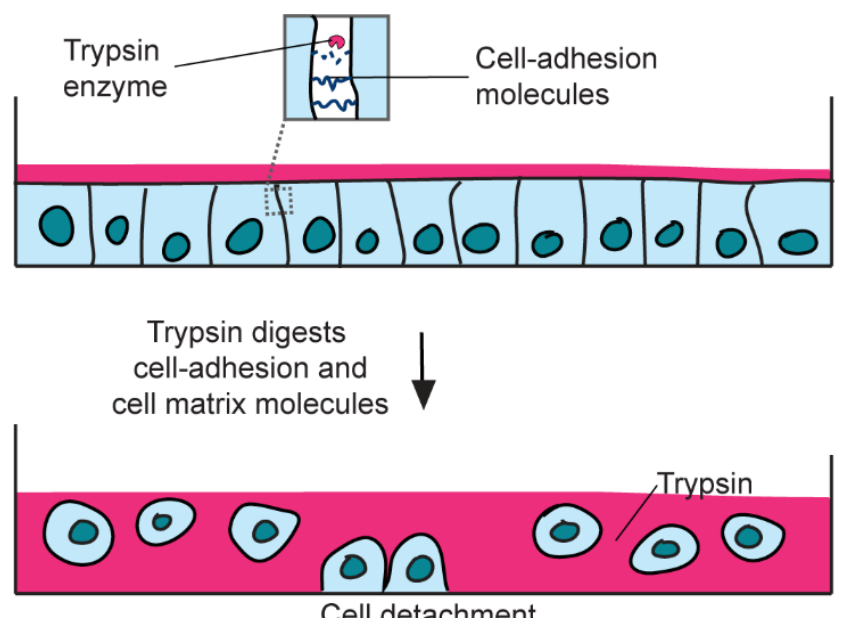

Figure 6. Effect of trypsin on a cell monolayer. Trypsin causes cell detachment when applied to a monolayer of cells, by digesting the cellular adhesion and matrix molecules. Trypsinized cells appear rounded due to lack of attachment to substrate.

\subsubsection{Implications for the design of microfluidic sur-} gery

In bulk solution, the application of surfactant or enzymes inflicts an "injury" to the entire cell or population of cells. In order to inflict a local injury to a small, specific region on a cell membrane or cell population (e.g., a monolayer of cultured cells), the surfactant and enzyme solution would need to be applied at a length scale that is smaller than the size of the cell or the monolayer, ideally with high spatiotemporal precision.

To this end, microfluidics offers significant advantages due to laminar flow at low Reynolds numbers $(\operatorname{Re}<100) .{ }^{16}$ In this flow regime, viscous effects dominate over inertia. Mixing occurs primarily by diffusion. The Peclet number $\left(\mathrm{Pe}=\mathrm{v}^{*} \mathrm{w} / \mathrm{D}\right.$ $=\mathrm{Z} / \mathrm{w}$, where $\mathrm{v}=$ velocity, $\mathrm{w}=$ channel width, $\mathrm{D}=$ diffusion constant, and $\mathrm{Z}=$ channel length at which mixing is complete), characterizing the relative importance of advection to diffusion, can be quite high.

As an example, a stream of surfactant or enzyme solution can be sandwiched between two buffer streams to flow over a monolayer of cells to confine the region of injury. The width of the injured region is determined by the width of the laminar stream of surfactant or enzyme, which is in turn governed by diffusion of the surfactant or enzyme molecules. The diffusion coefficients of SDS, Triton X-100, and trypsin are $\sim 10^{-6} \mathrm{~cm}^{2} / \mathrm{s}$, $10^{-7} \mathrm{~cm}^{2} / \mathrm{s}, \sim 10^{-6} \mathrm{~cm}^{2} / \mathrm{s}$, respectively. ${ }^{93,94}$ To maintain a stream width of $\mathrm{w}=10 \mu \mathrm{m}$ for a length $\mathrm{Z}=1 \mathrm{~cm}$, or a $\mathrm{Pe}=1000$, the flow velocities needed are $\sim 1 \mathrm{~cm} / \mathrm{s}, 0.1 \mathrm{~cm} / \mathrm{s}$, and $1 \mathrm{~cm} / \mathrm{s}$, respectively.

These flow rates are easily achievable in typical microfluidics systems. Therefore, the use of laminar flow allows spatial control of the injury by chemical methods with high precision. Furthermore, the stream of surfactants or enzymes can be switched to a buffer at a later time point to stop the injury and observe subsequent healing or regeneration events. The temporal resolution is limited by the mass transport of changing to a solution with a different composition, and is typically on the order of seconds.

\subsubsection{Electrical methods}

Electroporation utilizes an electric field to permeabilize the plasma membrane of a cell, typically for the transport of cargo in and out of the cell. ${ }^{95,96}$ Cells in their natural state maintain an electrical potential difference, the transmembrane voltage, across their plasma membranes. ${ }^{96}$ In eukaryotic cells, the transmembrane voltage ranges from $-40 \mathrm{mV}$ to $-70 \mathrm{mV}$. Application of an external electric field causes the transmembrane voltage to increase, resulting in changes in the structure and composition of the lipid bilayer. Beyond a critical induced transmembrane voltage, the plasma membrane becomes permeabilized (Figure 7). The critical transmembrane voltage required for membrane permeabilization depends on several factors including the size and the type of the cell, the curvature of the plasma membrane and the exposure duration. ${ }^{96}$ In Chinese hamster ovary cells, permeabilization of the membrane was found to occur when the transmembrane voltage reached $240 \mathrm{mV}$ and the electric field necessary to permeabilize the membrane was around $0.3 \mathrm{kV} / \mathrm{cm} .{ }^{97}$ The size of the pore created during electroporation is usually of the order of nanometers. The pore seals spontaneously over a timescale of seconds to minutes post electroporation. However, if the induced transmembrane voltage is 3-5 times the critical threshold, there can be irreversible damage to the plasma membrane. ${ }^{96}$

\subsubsection{Implications for the design of microfluidic sur-} gery

The fabrication and integration of electrodes consisting of different materials in a microfluidic system has been demonstrated successfully for a range of applications. ${ }^{98-100}$ Electroporation requires a high electric field for plasma membrane permeabilization. Such high electric fields can be achieved easily in microfluidic systems by applying a small voltage across a device with dimensions that can be as small as the size of a cell (tens of micrometers). For example, to generate an electric field of $1 \mathrm{kV} / \mathrm{cm}$, a voltage of only $1 \mathrm{~V}$ is needed across a $10 \mu \mathrm{m}$ space. In addition, the integration of cell cultures on an array of electrodes, or a continuous flow of suspended cells between two electrodes in a microfluidic system makes it possible to electroporate a large number of cells at a time in a high throughput manner.

\subsection{Methods to fuse}

Most microfluidic fusion surgery have focused on the fusion between cells. Cell fusion involves the coalescence of cell membranes of two cells into one. A generally accepted model of cell fusion is the stalk-pore model (Figure 8). ${ }^{101-103}$ In this model, fusion initiates when the lipids in the outer leaflets of the plasma membrane connect, which can be mediated by the interactions between the polar head groups of the phospholipids in the outer leaflets through receptor-ligand interactions. The fusion of the outer leaflets forms an intermediate stalk. Radial expansion of the stalk induces a transient hemifusion diaphragm, which is a local bilayer made of the two initial inner leaflets. A fusion pore is formed in the diaphragm after the inner leaflets merge. Finally, the pore expands and leads to complete fusion.

In vivo, cell fusion is typically mediated by fusogen proteins. ${ }^{104}$ Given the biotechnological applications of fused cells (see Section 6), external forces have been applied to induce 

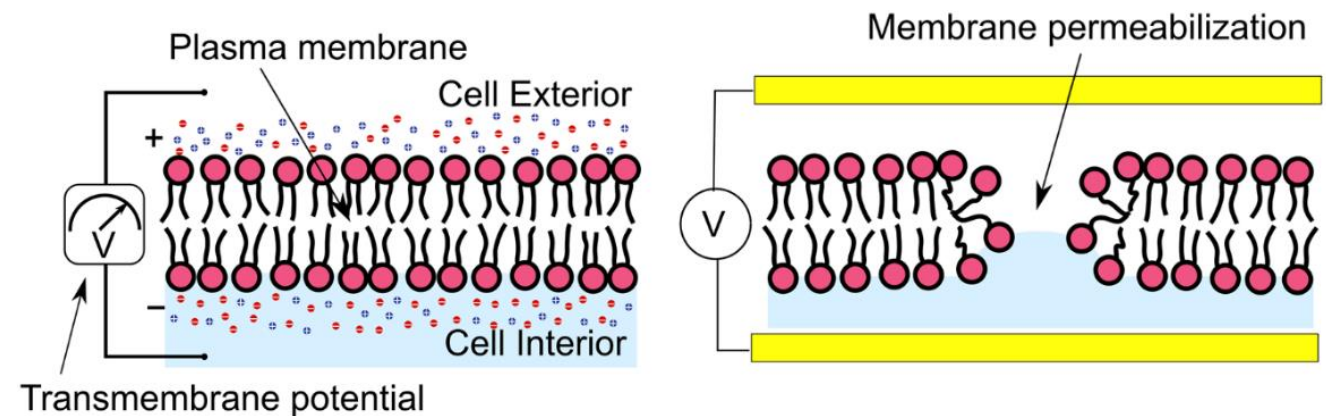

Figure 7. Electroporation. Schematic diagram showing the process of plasma membrane disruption by the principle of electroporation.

cell fusion involving biological, chemical, and electrical methods. Below, we will summarize the mechanisms of the most common methods for inducing cell fusion in microfluidics: virus-mediated fusion, polyethylene glycol-mediated fusion, and electrofusion (Figure 9). ${ }^{105}$

\subsubsection{Virus-mediated fusion}

Many viruses have been reported to be capable of fusing human cells. ${ }^{106}$ Sendai virus, a mouse respirovirus, also called the hemagglutinating virus of Japan (HVJ), is a popular virus used for in vitro cell fusion. It is infectious to many cancer cell lines, and has been used to fuse cells to form hybridoma for monoclonal antibody production. ${ }^{107}$ In addition to Sendai virus, a number of viruses have been used for cell fusion. ${ }^{108}$

There are two primary mechanisms through which viruses fuse cells. ${ }^{106,109}$ The first mechanism involves infecting a cell and causing the infected cell to express fusogenic proteins on the plasma membrane. The infected cell can then fuse with another cell having a receptor for the fusogenic protein, by inducing membrane rearrangement and expanding the fusion pore. The second mechanism does not involve the infection of the cells by the virus. Rather, the virus particles bridge two cells together, and mediate the modification of membrane structure thereby allowing fusion to occur. ${ }^{10}$

Virus-mediated fusion depends on the concentration of the virus, $\mathrm{pH}$, temperature, and incubation time. For the $\mathrm{HVJ}$ mediated fusion of Ehrlich ascites tumor (EAT) cells, viral infection occurs at 1200 to 3600 virus particles per cell at neutral $\mathrm{pH} .{ }^{111}$ Cells are treated with virus aggregate at $0{ }^{\circ} \mathrm{C}$ and fusion occurs at $37^{\circ} \mathrm{C}$ within $\sim 5$ minutes.

A challenge of using viruses for cell fusion is that the experimental parameters must be tailored to different cells. Additionally, virus-mediated fusion is limited to cell types with appropriate receptors. Given these limitations, virus-mediated fusion is now less common after the discovery of PEGmediated fusion and electrofusion. ${ }^{112}$

\subsubsection{Polyethylene glycol (PEG)-mediated fusion}

Polyethylene glycol (PEG)-mediated fusion is the most widely used reagent for chemical fusion by addition to cell suspensions. ${ }^{113-115}$ PEG is a hydrophilic linear polymer of ethylene oxide with a hydroxyl terminal.

The mechanism by which PEG fuses cells is still not fully understood. ${ }^{116-118}$ However, PEG-mediated fusion is believed to occur through the aggregation of the cells to be fused and the destabilization of the lipid bilayer. Cell aggregation with
PEG is attributed to PEG's high affinity for water, causing the depletion of water from solution. ${ }^{119}$ This dehydration causes an elevation of osmotic pressure that regulates the amount of water between cells, thereby creating an attractive depletion force driving cell aggregation. ${ }^{117,118,120}$ PEG destabilization of the bilayer is caused by the dehydration of water molecules interacting with head groups of the bilayer as described in vesicle-fusion studies. ${ }^{121,122}$ Agents that enhance or alleviate the dehydration-induced stress to the cell membrane will favor or inhibit fusion. ${ }^{123}$

The PEG used for fusion typically has a molecular weight between 1000 and $10000 .{ }^{118}$ The advantages of PEG are that it is water soluble, easy to handle, and has relatively low toxicity to cells at the optimal concentrations. However, the fusion efficiency in bulk is low (around $8 \%$ only). ${ }^{124}$ Importantly, the window for PEG concentrations that promotes cell fusion is narrow. PEG concentrations beyond a threshold level cause severe toxicity to cells. ${ }^{125-128}$ In a study on the effect of PEG concentration on the hybridization of 3T3 and RG6 cells, it was found that the exposure to PEG at a concentration of around $50 \%$ for 1 minute led to the highest yield of hybrids. ${ }^{115}$ Beyond this concentration, the yield decreased significantly, attributed in part to an overall decrease in cell viability.

\subsubsection{Electrofusion}

The electrical method for cell-cell fusion is also called electrofusion. Electrofusion was first described by Zimmerman in $1981 .^{129}$ The process starts with the application of an electric field $(\sim 100 \mathrm{~V} / \mathrm{cm})$ to pair cells and keep them in contact using dielectrophoresis. Next, the application of pulses of high electric field $(\sim \mathrm{kV} / \mathrm{cm})$ causes electroporation of the cell membranes. Subsequently, the pores expand and the membranes of the cells in contact fuse allowing cytoplasmic exchange. ${ }^{130}$

The factors affecting fusion yield include the electric field strength $\mathrm{E}_{0}$, pulse width $\mathrm{t}_{\mathrm{s}}$, the total duration of application $\Sigma \mathrm{t}_{\mathrm{s}}$, and the waveform of the electric field. ${ }^{131}$ Higher yields of fusion have been achieved using a square pulse than an exponential pulse with the same $\mathrm{E}_{0}$ and $\mathrm{t}_{\mathrm{s}}{ }^{131}$ Additionally, the fusion rate increases with $\mathrm{E}_{0}$ and decreases with temperature.

Electrofusion experiments are traditionally performed in cell suspensions. The fusion efficiency is low, ranging from $0.7 \%$ to $5.7 \%,{ }^{132}$ which has been attributed to random cell alignment and the lack of tools to retrieve hybrids from unfused parents. 


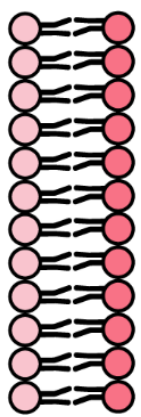

Cell 1

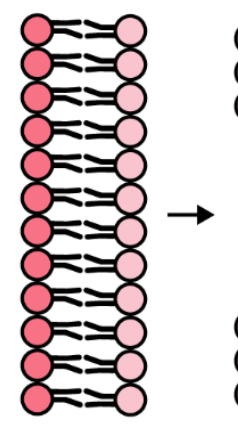

Cell 2

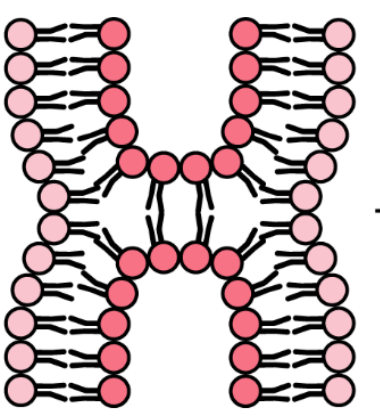

Stalk
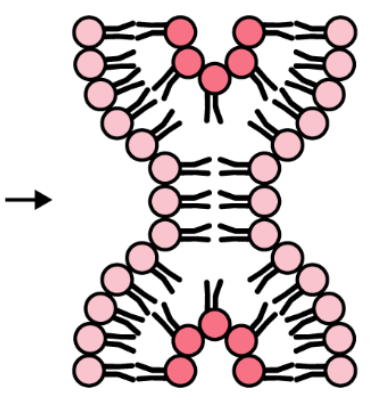

Hemifusion

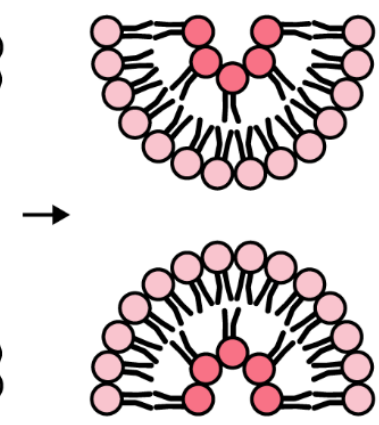

Pore

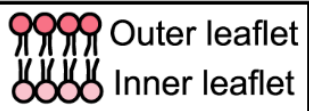

Figure 8. Stock pore model for fusion. This model describes the steps leading to the fusion of separate cells. First, the two cells that fuse are in close proximity. Then, stimuli promote the outer leaflet of each cell to mix and form a "stalk". The formation of the stalk promotes the fusion of the inner leaflet of the bilayer completing partial fusion or hemifusion. Finally, the more the inner leaflets mix a fusion pore forms allowing for cytoplasmic exchange completing the fusion process.
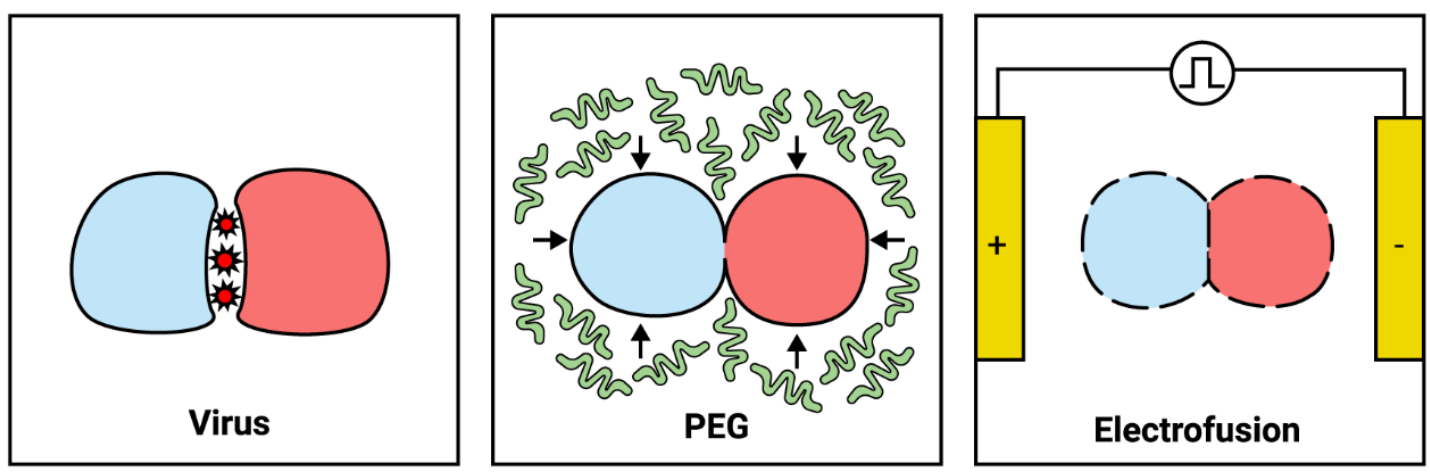

Figure 9. Common fusion methods. Schematic showing the three most commonly used methods for fusion in microfluidic devices.

\subsubsection{Implications for the design of microfluidic surgery}

One of the biggest challenges in cell fusion in bulk solution is the difficulty in pairing cells and ensuring their contact prior to the application of the fusing agents. The ability to control the flow and the trapping of single cells in microfluidics is thus of great value to fusion surgery. In addition, if the fusing agents are in solution (e.g., virus or PEG), they can be applied and washed off the cells with high temporal precision in microfluidics to maximize fusion yield and minimize cytotoxicity (if any). The simple integration with electrodes in a microfluidic system, as discussed above, also makes microfluidic systems amenable to electrofusion.

\subsection{Methods to transport}

In single-cell biopsy, the materials to be extracted, such as organelles and biomolecules, are in the micrometer to nanometer range. Their extraction thus requires transport mechanisms that differ significantly from macroscopic biopsies. Microfluidic techniques have emerged as a powerful tool enabling efficient extraction from within the cell. In this section, we will summarize principles of micro/nanoscale fluid flow relevant for single-cell biopsy.

\subsubsection{Pressure-driven flow}

A common way to drive fluid flow is by applying a pressure gradient across a pipe, channel, or other conduits (Figure $10 \mathrm{~A})$. In the microscale, viscous effects dominate and the flow is typically laminar. The flow exerts hydrodynamic drag on particles present and causes them to move. As the diameter of the conduit decreases, however, the pressure $\Delta P$ needed to drive the flow increases significantly as it scales inversely with the $4^{\text {th }}$ power of the radius $R$ as given by the Poiseuille equation (Eq. 3),

$$
\Delta P=\frac{8 \eta L Q}{\pi R^{4}} \quad \text { (Eq. 3) }
$$

where $\eta$ is the viscosity of the fluid, $L$ is the length of the pipe, and $Q$ is the volumetric flow rate. For example, a nano-conduit with a diameter $D_{c}=100 \mathrm{~nm}$ and a length $L_{c}=10 \mu \mathrm{m}$ would require a pressure difference $\Delta P \sim 10^{-1}-10^{1}$ atmospheres to drive the flow of cytosol (with a viscosity assumed to be 1 $10^{2}$ times higher than water $)^{133,134}$ at an average velocity $u \sim 1$ $\mathrm{mm} / \mathrm{s}$, or approximately $1 \%$ of cell volume per second if the cell is assumed to have a size of $\sim 10 \mu \mathrm{m}$. 


\subsubsection{Electrokinetic flow}

An alternative way to drive fluid flow is by applying an electric field. This type of flow is also known as electrokinetic flow (Figure 10B).

\subsubsection{Electroosmosis}

Electroosmotic flow of an electrolyte inside a micro/nanofluidic channel is generated by application of an electric field across the channel. ${ }^{18,135,136}$ An electrical double layer is formed at the interface between a solid surface and an electrolyte solution due to electrostatic attraction between the free ions in the electrolyte (counterions) and the bound surface charges on the solid surface (Figure 10B). The bulk electrolyte thus gains a net charge. When an external electric field is applied across the electrolyte, the net charge in the bulk electrolyte generates a fluid flow due to the movement of the ions. The electroosmotic velocity profile depends on the thickness of the electrical double layer, applied potential difference and the material properties of the electrolyte (electrical permittivity and viscosity). The thickness of the electrical double layer, or the Debye length, is a function of the ionic strength of the electrolyte, and is typically on the order of angstroms (at high electrolyte concentration) to nanometers (at low electrolyte concentration). For example, for a $5 \mathrm{mM}$ ammonium acetate solution, the Debye length is $\sim 4 \mathrm{~nm}$. In the limit that the channel is wide compared with the Debye length, the electroosmotic flow can be approximated as a plug flow with the velocity $u_{E O F}$ given by the classic Helmholtz-Smoluchowski equation (Eq. 4),

$$
u_{E O F}=\frac{\varepsilon \xi}{\eta} E
$$

where $\varepsilon$ is the permittivity of the solution, $\xi$ is the zeta potential, $\eta$ is the dynamic viscosity of the electrolyte, and $E$ is the electric field applied. In the nano-conduit example above (diameter $100 \mathrm{~nm}$, length $10 \mu \mathrm{m}$ ), assuming the material is glass $(\xi \sim-50 \mathrm{mV})$ and the nanopipette is filled with a 1:1 electrolyte (5 mM ammonium acetate), an electric field $\mathrm{E} \sim 10^{4} \mathrm{~V} / \mathrm{m}$ would be needed to drive the flow at $u_{E O F} \sim 1 \mathrm{~mm} / \mathrm{s}$.

\subsubsection{Electrophoresis}

Electrophoresis is the transport of charged particles in an electrolyte in the presence of an external electric field. ${ }^{136,137}$ Like electroosmosis, electrophoresis is brought about by the formation of an electrical double layer at the interface between the electrolyte and the particle. A charged particle surrounded by a diffuse layer of counterions moving in an electrolyte solution experiences an electrical force due to the applied electric field, and the drag force due to the relative motion between the fluid and the particle (Figure 10B). ${ }^{137}$ The resulting velocity of the particle, $u_{E P}$, is proportional to the electric field and the electrophoretic mobility of the particle (Eq. 5). ${ }^{138}$

$$
u_{E P}=\mu_{E P} E \quad \text { (Eq. 5) }
$$

The electrophoretic mobility depends on the zeta potential and the permittivity of the particle, as well as the viscosity of the fluid, but the detailed expression depends on the shape and the size of the particle relative to the Debye length. ${ }^{138}$ As an example, hexanucleotide ssRNA homopolymers of polyadenylic acid have an estimated electrophoretic mobility $\mu_{E P}$ of $4 \times 10^{-8} \mathrm{~V}^{-1} \mathrm{~m}^{2} \mathrm{~s}^{-1}$ in an aqueous electrolyte. ${ }^{139}$ To achieve an electrophoretic velocity $u_{E P} \sim 1 \mathrm{~mm} / \mathrm{s}$ in an aqueous electrolyte (e.g. ammonium acetate), an electric field $E \sim 10^{4} \mathrm{~V} / \mathrm{m}$ would be needed.
A Pressure driven flow

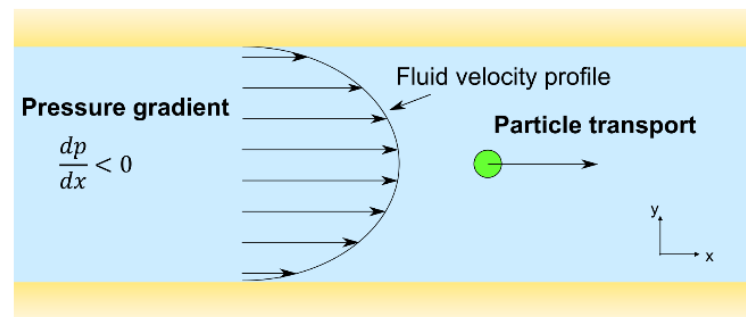

B Electrokinetic flows

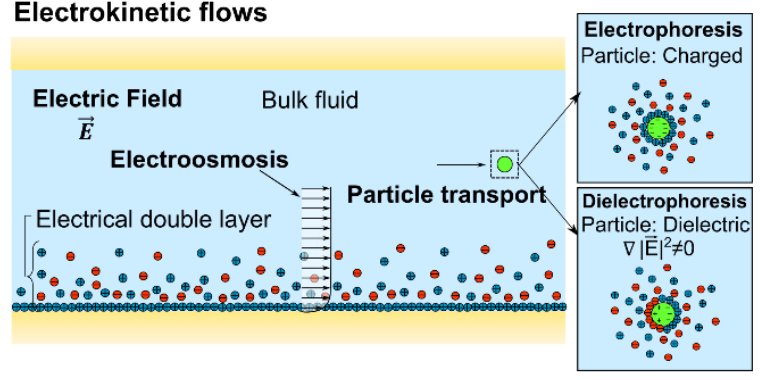

C Electrowetting

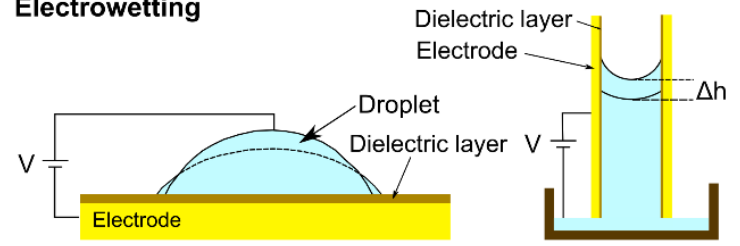

D Transport by diffusion

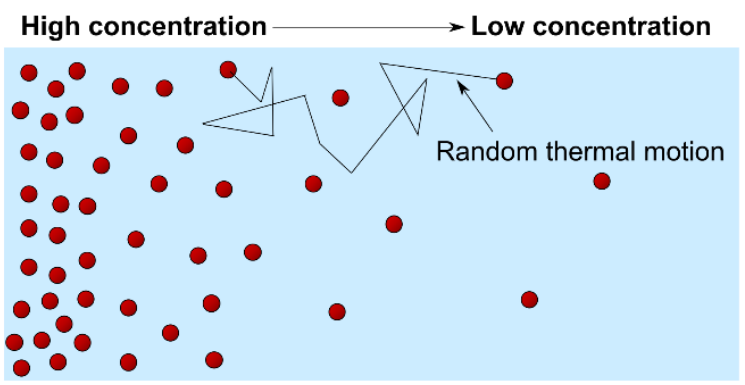

Figure 10. Methods to transport cellular contents. Schematic diagrams showing (A) pressure-driven flow, (B) electrokinetic flows, (C) electrowetting, and (D) diffusion mediated transport.

\subsubsection{Dielectrophoresis}

Dielectrophoresis is the transport of neutral dielectric particles upon the application of a non-uniform electric field (Figure 10B). A dielectric particle placed in an electric field polarizes to form an electrical dipole. In a non-uniform electric field, the dipole experiences a net force due to the non-zero gradient of the electric field which causes the particle to move. ${ }^{140}$ The dielectrophoretic velocity is given by (Eq. 6), ${ }^{141}$

$$
u_{D E P}=\frac{a^{2} \varepsilon}{6 \eta} f_{C M} \nabla|E|^{2} \quad \text { (Eq. 6) }
$$

where $a$ is the radius of the particle, $\varepsilon$ and $\eta$ are the permittivity and dynamic viscosity of the fluid respectively, and $f_{C M}$ is the real part of the Clausius-Mossotti factor $\left(f_{C M}=\right.$ $\left.\operatorname{Re}\left[\frac{\hat{\varepsilon}_{p}-\hat{\varepsilon}}{\hat{\varepsilon}_{p}+2 \hat{\varepsilon}}\right]\right)$, where $\hat{\epsilon}_{p}$ and $\hat{\varepsilon}$ are the frequency-dependent complex permittivities of the particle and the fluid respectively. Given this relationship, larger particles experience a higher dielectrophoretic force and move faster in a given electric field 
gradient than smaller particles. Therefore, dielectrophoresis offers a way to transport particles based on their size, making it possible to extract specific molecules from the cell during biopsy.

For example, for the trapping of a DNA molecule at the tip of a nanotweezer, a force of $\sim 10 \mathrm{fN}$ was found to be needed to overcome Brownian motion. ${ }^{142}$ This force was achieved by applying an electric field gradient $\left.|\nabla| E\right|^{2} \mid>2.5 \times 10^{16} \mathrm{~V}^{2} / \mathrm{m}^{3} .{ }^{143}$ At this electric field gradient, assuming the size of the molecules is $\sim 10 \mathrm{~nm}$ and $f_{C M}$ is on the order of $1,{ }^{144,145}$ the velocity of the molecule $u_{D E P} \sim 10^{2}-10^{0} \mathrm{~nm} / \mathrm{s}$ for a cytosol viscosity 1 $-10^{2}$ times that of water.

\subsubsection{Electrowetting}

The wetting characteristics at a solid-liquid interface depend on the balance between the cohesive forces between the liquid molecules at the interface and the adhesion between liquid molecules and the surface of the solid. A liquid droplet wets the solid surface more if the adhesive forces are higher than the cohesive forces, and vice versa. The degree of wetting is characterized by the contact angle at the interface and is related to the interfacial tensions by the Young-Dupre equation. Application of an electric field between the two phases induces charges at the solid-liquid interface, thereby modulating the contact angle, which can in turn drive fluid flow (Figure 10C). ${ }^{146}$ For example, in a $100 \mathrm{~nm}$ diameter glass capillary filled with water, assuming the surface force is only countered by gravity, we can approximate the change in the height $h$ of the water column using the capillary rise equation (Eq. 7),

$$
\frac{d h}{d \theta}=-\frac{2 \gamma_{L G} \sin \theta_{0}}{\rho g r} \quad \text { (Eq. 7) }
$$

where $\gamma_{L G}$ is the liquid-gas interfacial tension, $\theta_{0}$ is the initial contact angle of water on glass, $\rho$ is the density of water, and $r$ is the radius of the capillary. A $0.1^{\circ}$ change in the contact angle (assuming $\left.\theta_{0}=15^{\circ}\right)$ would result in $\sim 1 \mathrm{pL}(\sim 1$ cell volume) change in volume of water inside the capillary. The voltage needed to obtain this change in contact angle is given by the Young-Lipmann equation (Eq. 8),

$$
\left.V=\sqrt{\frac{2 \gamma_{L G} d}{\epsilon}\left(\cos \theta-\cos \theta_{0}\right)} \quad \text { (Eq. } 8\right)
$$

where $\varepsilon$ is the permittivity of water, and the dielectric layer is assumed to have a thickness $d=10 \mathrm{~nm}$ between the electrode and the liquid. The voltage necessary for a $0.1^{\circ}$ change in the contact angle is thus only $\sim 10^{-2} \mathrm{~V}$.

\subsubsection{Diffusion-mediated transport}

At small length scales, the effect of the thermal energy of molecules is significant. The movement of molecules at such length scales is dictated, to a large extent, by diffusion down a concentration gradient (Figure 10D). Diffusion-mediated transport from cells requires disruption of the plasma membrane which can be achieved by methods described in Section 2.1. The downside of diffusion is that it is slow. The coefficient of diffusion, given by the Stokes-Einstein equation, $D=$ $\frac{k_{B} T}{6 \pi \eta r}$ ( $k_{B}$ is the Boltzmann constant, $T$ is the temperature), depends on the viscosity of the liquid $\eta$ and the size $r$ of the particle being transported. ${ }^{133}$ Let us consider hexanucleotide ssRNA homopolymers of polyadenylic acid which has an estimated coefficient of diffusion in water of $\sim 0.24 \times 10^{-9} \mathrm{~m}^{2} / \mathrm{s} .{ }^{139}$ The time scale $t$ for diffusing across the length $L_{c}$ of the nano- conduit scales as $t \sim \frac{L^{2}}{D} \sim 10^{-1}$ to $10^{1}$ seconds, assuming the viscosity of the cytosol is $1-10^{2}$ times that of water.

\subsubsection{Implications for the design of microfluidic surgery}

In order to minimize damage to cells, the opening in the plasma membrane caused by the biopsy process should be as small as possible, ideally less than hundreds of nanometers. With existing fabrication techniques, it is possible to fabricate nanochannels, pipettes, or other conduits with a diameter of this size range (see details in Section 5).

As can be seen from the examples on the nano-conduit above, one of the challenges of using conduits with such a small diameter is the increasing difficulty in transporting fluids using some of the common transport mechanisms such as pressure driven flow, given the exceedingly large pressure that may be required depending on the viscosity of the cytosol. In practical devices that rely on pressure driven flow, the nanoconduit can be tapered such that only the tip is small, but the diameter of the rest of the conduit is in the tens of micrometers range.

Comparing different electrokinetic transport mechanisms, electrowetting is advantageous in that only a very small voltage is needed to cause a significant change in the capillary height and thus the volume of fluid aspirated into the conduit. Indeed, in devices that apply an electric field across the conduit, electrowetting dominates over electroosmosis and electrophoresis in driving the flow (see details in Section 5). The velocity of molecules driven by dielectrophoresis is small. As such, most applications of dielectrophoresis are for local trapping of molecules (or cells, see Section 6), rather than for transport over long distances.

For transport based on the diffusion of molecules down a concentration gradient, while it is simple, the process is slow compared with other transport mechanisms. It may still be suitable for probing cellular processes that span long time scales (tens of minutes). However, the extraction of larger organelles like mitochondria will be very difficult using diffusion alone. 


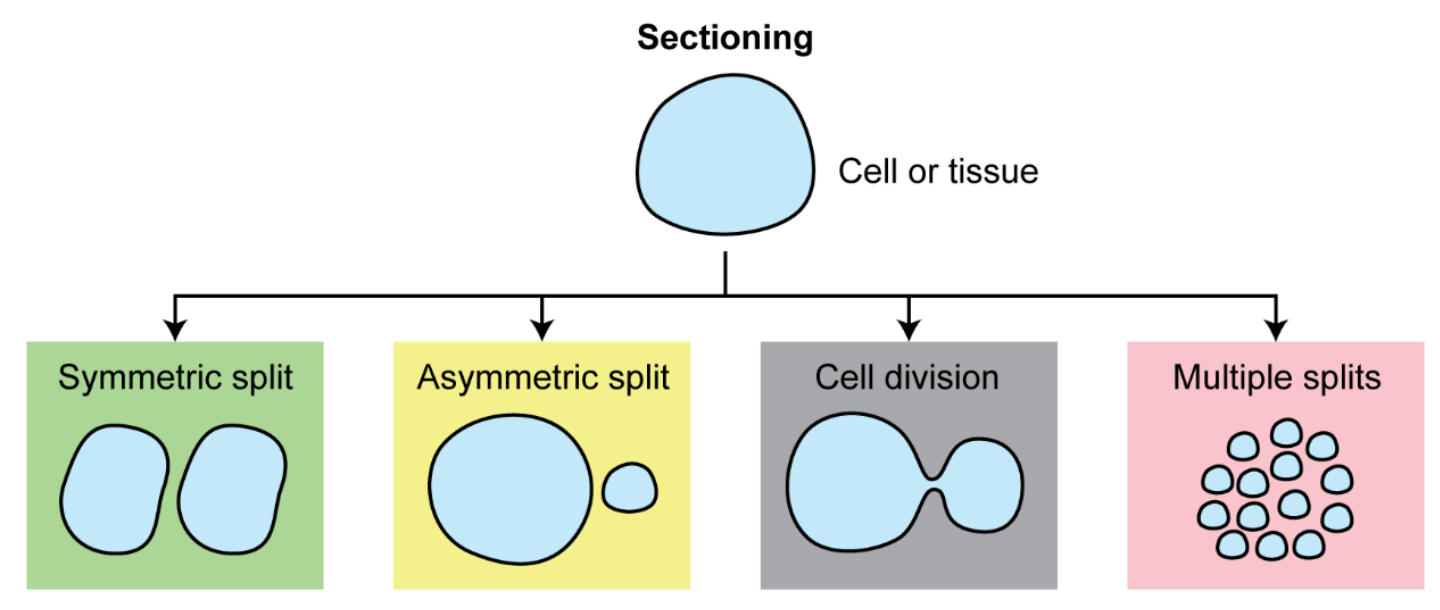

Figure 11. Types of sectioning surgery. In a sectioning surgery, a cell or tissue sample may be split symmetrically into two halves, asymmetrically into two pieces, during cell division, or into multiple pieces (listed from left to right).

\section{SECTIONING}

We define sectioning as the process of dividing or separating an entity physically into multiple distinct parts, or sections. This process can be applied in various forms, including splitting an entity into two symmetric halves, into two asymmetric parts, or into many fragments, and even facilitating cell division (Figure 11). The multiple pieces generated can be used in downstream experiments or analysis, including histology, microscopy, sequencing, drug screening, wound healing, and regeneration studies as described below.

In these studies, microfluidics can facilitate the handling and analysis of samples that have already been sectioned outside the device using existing methods such as the microtome or tissue chopper. The advantages of microfluidics in such use cases have been demonstrated in applications such as spatial transcriptomics and cancer drug screening. ${ }^{147-150}$ Alternatively, microfluidic methods can be used to perform the sectioning procedure itself. Here, we will focus on describing the microfluidic methods to perform the sectioning procedure at the single cell, dividing cell, and multicellular scales (Figure 12). We will consider a microfluidic device to have performed sectioning surgery when the sample is kept alive during the sectioning, and when the microfluidic design is an integral component of the sectioning process.

\subsection{Single cells}

At the single-cell level, sectioning involves splitting a cell into two or more subcellular fragments. Two key applications demonstrated so far are to generate enucleated cells, and to study wound repair and regeneration capabilities at the single cell level. We note that these operations are distinct from cell lysing in that the subcellular fragments remain viable.

\subsubsection{Enucleation}

High quality enucleated cells are crucial for cell reprogramming and cloning via somatic cell nuclear transfer. Somatic cell nuclear transfer involves the removal of a donor nucleus from a cell and transferring it into an enucleated receptor cell. ${ }^{151}$ It is typically performed by mechanical separation via a micropipette. ${ }^{152}$ Compared with other methods (e.g., drug and/or UV treatment), mechanical separation has yielded the highest viability of enucleated cells. ${ }^{152}$ However, the process suffers from low efficiency and reproducibility. Manual methods require $>1$ min per cell (not including locating or retrieving the sample) and suffer from high variability (standard deviation of $\pm 30 \%$ of original total volume removed) and low viability $(\sim 40 \%) .{ }^{153}$ While efforts have been made to use robotically controlled micropipettes and/or computer vision to automate and enhance processes such as enucleation, ${ }^{153-155}$ microfluidic methods offer a unique advantage in facilitating high precision and high throughput enucleation procedures.

The first approach of enucleation is to use solid tools to separate and pinch off the nucleus from the oocyte mechanically inside a microfluidic channel. Magnetically driven microtools (MMTs) can generate forces on the order of millinewtons. An early strategy to enucleate oocytes in microfluidics used two MMTs inside a microfluidic device. ${ }^{156,157}$ The MMT consisted of a $200 \mu \mathrm{m}$ thick, $\sim 1 \mathrm{~mm}$ long blade with a tip that was approximately $20 \mu \mathrm{m}$ wide which tapered to $<100 \mu \mathrm{m}$ wide in the body. The base of the MMT contained large circular regions plated with $\mathrm{Ni}$ for magnetization. The rest of the MMT was fabricated in Si for biocompatibility. Each MMT was sealed inside a microfluidic channel and controlled by permanent magnets attached to a linear actuator that were placed outside the glass bottom of the microfluidic device. To reduce friction between the MMTs and the glass, the surface of the MMT was etched with small $8 \mu \mathrm{m}$ wide grooves, which reduced the contact area with the glass. The glass was vibrated by an ultrasonic piezoelectric ceramic to further reduce the friction. The reduced friction lowered the lag between the actuator and the true device position, which enabled a $1.1 \mu \mathrm{m}$ precision in positioning the MMT tip.

Swine oocytes used were $\sim 100 \mu \mathrm{m}$ in diameter. To enucleate the oocytes, the oocytes first flowed into a chamber containing the MMT tips. The two MMTs rotated the oocyte into position and cut off the nucleus portion, and the nucleus portion and the enucleated oocyte then flowed into separate outlet channels. The device performed the cutting process in $\sim 10 \mathrm{~s}$, not including manipulating or flowing the oocyte. The device succeeded in removing an average of $20 \%$ of the original total cell volume, which was a significant improvement from manual methods. 


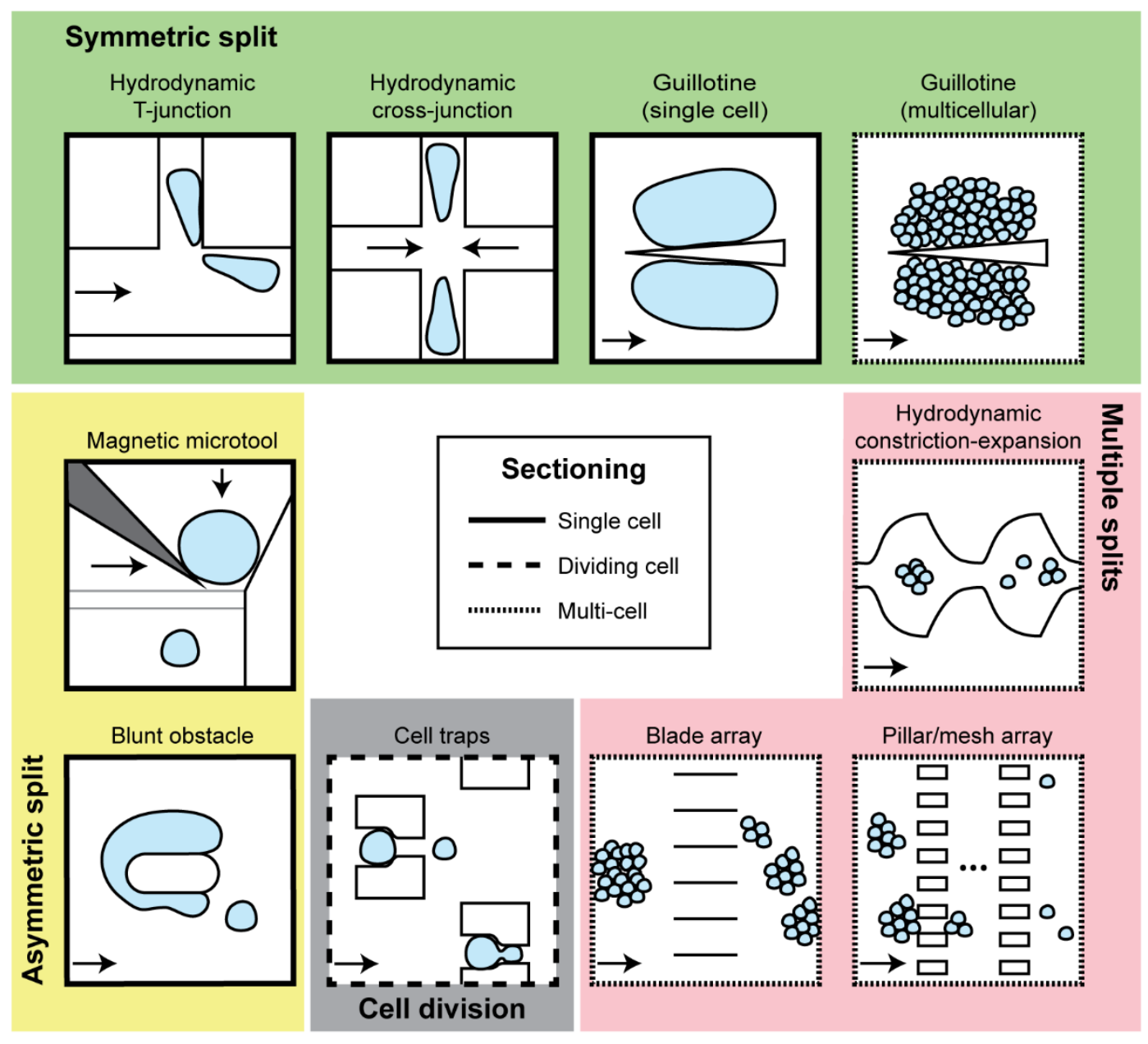

Figure 12. Summary of sectioning procedures. Methods are sorted within the four categories of sectioning: (1) symmetric split, (2) asymmetric split, (3) cell division, and (4) multiple splits. The operating biological scale of each method is indicated by the method schematic border. Arrows indicate direction of flow and tool movement (if applicable).

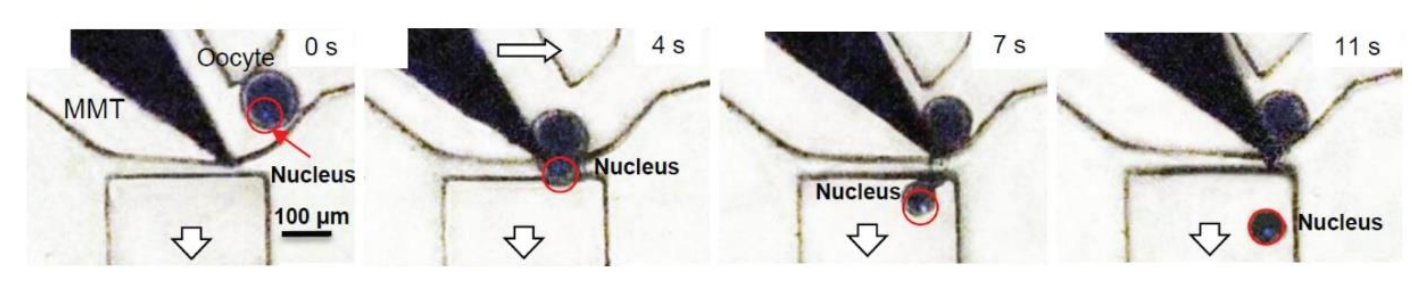

Figure 13. Application of microfluidics to cell enucleation. Magnetic microtool for enucleation. The microtool positions the oocyte against a ridge. The nucleus-containing portion of the oocyte is extruded under the ridge, and the microtool pushes against the opposite wall to complete the cut. Reproduced from Feng et al. under a Creative Commons BY 4.0 License. ${ }^{158}$

A follow-up study used the same MMT design, but only used a single MMT for bovine oocytes ( 100 $\mu$ m diameter).$^{158}$ The change to a single MMT was made to avoid sticking of the MMT caused by cutting the oocyte. Instead, the MMT pinned the oocyte against a ridge and the wall of the device, and hydrodynamic forces extruded the nucleus-containing portion under the ridge and through the small gap between the MMT tip and the wall (Figure 13). The MMT was pressed fully against the wall to cut the cell and complete the enucleation. The size of the removed portion of cytoplasm was controllable by the time between pinning the oocyte and completing the cut. The improved design demonstrated a cutting time of $2.5 \mathrm{~s}$ when removing $20 \%$ of the original total cell volume, not including manipulating or flowing the oocyte. Both the enucleated oocyte and the removed nucleus portion flowed into the same outlet for later sorting. The downstream viability 
was not reported for either of these MMT studies, so the true advantage of microfluidic enucleation using MMTs compared with manual or robotically-assisted methods remains to be seen.

A second approach to enucleate cells uses hydrodynamic forces generated by a flow in a microfluidic $\mathrm{T}$-junction to remove the nucleus-containing portion of the oocyte. ${ }^{159-161}$ In these studies, bovine oocytes softened by an actin depolymerizing drug were aspirated into a thin microchannel, and hydrodynamic forces sheared the cell into two pieces. The channel consisted of a straight channel $(700 \mu \mathrm{m}$ wide x $200 \mu \mathrm{m}$ tall $)$, with a side channel $(200 \mu \mathrm{m}$ wide $\mathrm{x} 50 \mu \mathrm{m}$ tall $)$ oriented orthogonal to the straight channel for aspiration. ${ }^{161}$ The device contained an inlet for injecting the oocytes at a variable speed and an inlet for injecting the fluid at $25 \mu \mathrm{L} / \mathrm{min}$ for cutting ("cutting flow"). The oocyte injection speed was variable and controlled by a computer algorithm to position the cell at the aspiration channel. The cell was aspirated at $0.2 \mu \mathrm{L} / \mathrm{min}$ until half of the cell volume entered the aspiration channel and detected using image processing, at which point the cutting flow was turned on. The cells were split roughly in half by the cutting flow shearing the aspirated cell against the corner of the T-junction. The orientation of the oocyte was not controlled during this process and both cell halves exited from the outlet for downstream sorting. The enucleation process took $\sim 100 \mathrm{~s}$, not including aspiration or positioning time, but there is potential for improvement in the syringe pump control. The success rate of the enucleation process was $98 \%$, but the oocyte viability was not reported.

\subsubsection{Bisection}

Studying wound repair and regeneration in single cells can provide insight into cellular functions and related pathologies. Single-cell organisms such as Amoeba ${ }^{162}$ and Stentor coerule$u s^{163}$ have been studied for their regenerative properties upon major injury such as bisection. These systems have been studied traditionally by manual surgery using sharp needles, where the organism is sectioned into two or more small fragments. Due to the small size of these organisms $(<1 \mathrm{~mm})$, controlling the location of surgery requires much dexterity. The manual surgery may be performed by hand or with the assistance of a micromanipulator apparatus. The earliest apparatus for cutting single cells under a microscope was described by Schmidt in 1859 , consisting of various microneedles and tools that could be positioned via screw mechanisms and demonstrated by cutting liver cells into two halves. ${ }^{164}$ As discussed in Section 1.1, improvements were made by Barber and Chambers in the early 1900 s by inverting the dissection stage so that higher magnifications could be used..$^{5}$ Today, both forms of manual surgery (by hand or by using micromanipulators) are still used. ${ }^{165,166}$ The basic principles and methods have not changed significantly despite the limitations (requirement for dexterity, low reproducibility, and throughput).

Microfluidics has drastically increased the throughput and reproducibility of single cell sectioning. The high throughput is critical for mechanistic studies and analysis (e.g., RNA sequencing) that require many cells synchronized in the same stage of their repair and regeneration process.

An intuitive approach to cutting a single cell is to use a physical blade. This approach was realized in a microfluidic guillotine designed for single-cell bisection of Stentor coeruleus. The guillotine consisted of a stationary blade centered inside a microfluidic channel. ${ }^{6}$ The blade was a triangular wedge made of PDMS, which was found to be sufficiently stiff to cut the cell. The cells were introduced into the channel having a width smaller than the diameter of the cell to provide a tight confinement and alignment with the blade. Due to the limited sharpness of the blade as fabricated by soft lithography, the actual cutting process likely involved the deformation of the cell around the blade followed by pinch-off of the membrane. The extent of the wound inflicted on the cells was found to increase with an increasing applied flow rate. At a flow rate of $\sim 1 \mathrm{~cm} / \mathrm{s}$, the bisected fragments had a viability $>90 \%$. This viability decreased to $\sim 70 \%$ when the flow rate was increased to $\sim 6 \mathrm{~cm} / \mathrm{s}$. To increase the throughput, eight guillotines were used in parallel to achieve an overall throughput of $\sim 64$ cells/minute. To prevent the fouling of the blades from cell debris after cutting, cells were encapsulated inside water-in-oil droplets which facilitated the self-cleaning of the blades. Surface tension between the aqueous media and the oil facilitated the retaining of cell fragments within the drop, thereby preventing residues from accumulating on the knife. In a follow-up study, the microfluidic guillotine was used to wound a large number of cells, which were then analyzed from 4 seconds to 150 minutes post injury to evaluate the degree of wound closure, as well as the mechanical modes of wound response, at different time points. ${ }^{7}$ In these two studies, the blade had a vertical cutting edge since it was fabricated by conventional 2.5-dimensional (2.5D) photolithography. High resolution $3 \mathrm{D}$-printing methods, such as those employing twophoton polymerization, could enable the fabrication of blades with complex 3D geometries with improved cutting performance. ${ }^{167}$

Instead of a physical blade, it is also possible to bisect single cells using hydrodynamic forces. The hydrodynamic cell splitter contained a microfluidic cross junction that used extensional flow to stretch Stentor coeruleus until the cell split into two. ${ }^{168}$ Compared with the microfluidic guillotine, the hydrodynamic cell splitter operated at a higher throughput of $\sim 500$ cells/minute, an order of magnitude higher than the guillotine, while preserving high cell viability. Furthermore, since the sectioning procedure did not involve contact with any solid blades, there was no build-up of cellular debris. The device could be operated over long periods of time without any fouling issues.

\subsubsection{Fragmentation}

During cancer metastasis, cancer cells need to enter the microvasculature and can become fragmented due to fluid stresses. ${ }^{169,170}$ The ability of the cancer cell to resist fragmentation could thus be correlated to its metastatic potential. An in vitro device containing a blunt obstacle at the center of the microchannel was fabricated to observe the fragmentation behavior of prostate cancer cell lines. ${ }^{171}$ The gap between the obstacle and the sidewall of the channel was less than half of the cell diameter. The purpose of the obstacle was not to cut cells per se, but to induce cell deformation and the subsequent pinch-off of the cytoplasm. Using 10 parallel channels each containing an obstacle, the work was able to analyze the fragmentation probability, fragmentation time, and excess membrane area of over 60 cells. The experiments were conducted at a fixed pressure drop of $20 \mathrm{mmHg}$, which was within the range of intravascular pressure. The corresponding fluid shear stress in the 
gap between the obstacle and the sidewall was approximately $7 \mathrm{~Pa}$. The study found that the more invasive prostate cancer cell line (CL2) was more resistant to fragmentation than the less invasive cell line (LNCaP). ${ }^{171}$

\subsection{Cell division}

The study of cell division is of enormous interest to agerelated diseases and cancer. ${ }^{172,173}$ While cell division is a naturally occurring phenomenon, we consider it an application of microfluidic surgery when the device provides active intervention that facilitates cell division. This active intervention typically involves physically confining a mother cell in place and then separating the daughter cell away from the mother cell.

Studying the replicative lifespan of a cell, which is the number of cell divisions an individual cell undergoes before reaching senescence and then cell death, is fundamental to the biology of aging. Replicative lifespan studies have been performed traditionally in the yeast model Saccharomyces cerevisiae by seeding virgin daughter cells onto an agar plate, allowing them to divide, and using a micropipette to periodically remove the daughter cells while leaving the mother cell in place for observation. ${ }^{174,175}$ The daughter cells are initially smaller than the mother cells, and must be removed promptly to prevent nutrient depletion of the agar plate near the mother cell and before they grow to a size that is difficult to distinguish from the mother cell. Since the manual removal of daughter cells only occurs at discrete time intervals, excess nutrients may still be consumed by the daughter cells. Furthermore, for practical reasons, a maximum of $200-300$ mother cells can be studied per researcher in an experiment. Cells are also incubated at $4{ }^{\circ} \mathrm{C}$ overnight to slow cell division when researchers are not present, which increases the typical total time for a single experiment to $\sim 2-3$ weeks and may affect the results and interpretation. Clearly, these studies are limited by their low throughput and long experiment times, as well as by factors that may influence the cell biology.

Various microfluidic approaches have been developed to facilitate replicative aging studies in yeast. ${ }^{176-180}$ Generally, the key intervention provided by the microfluidic device is to keep the mother cell trapped while continually removing the daughter cells via fluid flow (Figure 14). Compared with the traditional method, microfluidic-facilitated replication lifespan studies achieved higher throughput (up to thousands vs. 200300 cells per experiment), required less time to complete (a few days vs. 2-3 weeks per experiment), and required significantly less manual labor. Furthermore, the microfluidic approach enabled continuous imaging of the trapped mother cells and also provided a constant microenvironment.

An early approach to trap mother cells was to seed yeast cells in microfluidic "jails". ${ }^{176}$ Cells were first loaded onto the open PDMS surface, and then the device was sealed in a custom screw assembly. These jails had various geometries, typically enclosing an area of $\sim 11 \mu \mathrm{m}$ width, and included several $\sim 4.5 \mu \mathrm{m}$ gaps in the walls. The daughter cells exited the jails through these gaps and were washed away by media flow. However, this device design had a loading efficiency of only $<25 \%$ and began to clog after a few generations due to inefficient daughter cell removal.

A later approach trapped mother cells using PDMS pillars. ${ }^{177,178}$ The pillars had a footprint of $\sim 40 \mu \mathrm{m} \mathrm{x} 40 \mu \mathrm{m}$ and had a gap approximately the size of a yeast cell $(\sim 4-5 \mu \mathrm{m})$ between the bottom of the pillar and the glass. When the cells were injected at $\sim 1000 \mu \mathrm{L} / \mathrm{h}$, the hydrostatic pressure expand- ed this gap, and when the injection was stopped, the pillars returned to their original position and trapped the yeast cells. A constant media flow of $\sim 100 \mu \mathrm{L} / \mathrm{h}$ provided fresh media and washed away the daughter cells, which were less likely to be trapped by the pillar due to their smaller size. This design supported up to $\sim 100-200$ pillars per array. In these studies, up to 4 arrays were placed in parallel and cells were observed for 40-60 hours. However, this trap design yielded a variable number of initial cells trapped per pillar, occasionally trapped the daughter cells, and had low retention rates (decreasing to a plateau $\sim 30 \%$ by 20 hours into the experiment).

More recent designs utilized an array of hydrodynamic traps that were optimized to hold a single yeast cell. ${ }^{179,180}$ The trap design was of critical importance as it ensured a single cell loading per trap, biased flow towards unoccupied traps to maximize efficiency, and forced daughter cells out of the trap where they would be removed by the media flow. Both hydro dynamic trap studies described here used flow rates of $\sim 5$ $\mu \mathrm{L} / \mathrm{min}$ for loading the cells and for providing continuous media flow. Crane et al. used a trap design consisting of two 5 $\mu \mathrm{m}$ long blocks angled at $90^{\circ}$, with a $3 \mu \mathrm{m}$ gap at the corner (Figure 14). In an array of 1500 traps, this trap design achieved $>90 \%$ loading efficiency. While the cell retention of the angled block traps was improved compared to the pillar trap design, with $\sim 75 \%$ cell retention at 20 hours compared to $30 \%$, the cell retention decreased to $\sim 60 \%$ by $\sim 50$ hours. An improved design on the hydrodynamic trap was achieved by using a deeper, cup-like trap shape. ${ }^{180}$ The cup had an opening of $6 \mu \mathrm{m}$, a depth of $\sim 6 \mu \mathrm{m}$, and a $3 \mu \mathrm{m}$ gap in the center. They achieved a $96 \%$ loading efficiency and $89-92 \%$ retention rate at 96 hours in a device containing $~ 8000$ traps, which was a far superior performance to any other trap design described here for replicative lifespan studies.

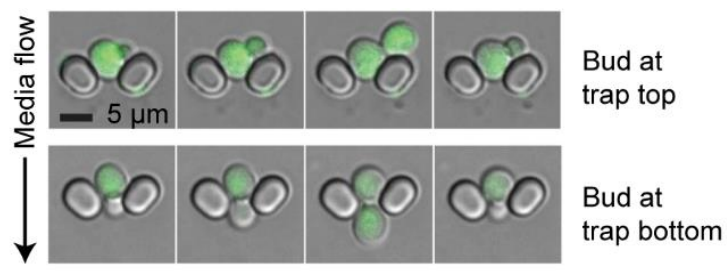

Figure 14. Application of microfluidics to cell division. Hydrodynamic trap where mother cells are held inside the trap by media flow, while daughter cells exit either through the top or bottom of the trap. Reproduced from Crane et al. under a Creative Commons BY 4.0 License. ${ }^{179}$

\subsection{Multicellular systems}

At the multicellular level, sectioning involves breaking a large tissue into its constituent single cells or into small tissue fragments. Applications include dissociating tissue into single cells for single-cell analysis, generating small tissue fragments for cell culture, and to study organoid regeneration.

\subsubsection{Dissociation into single cells}

For multicellular systems, single-cell analyses are powerful methods to better understand cell heterogeneity and its relation to biological function. ${ }^{181}$ For example, cell heterogeneity within a tumor is increasingly recognized to play an important role in understanding and treating cancer. ${ }^{182}$ In single-cell analysis 


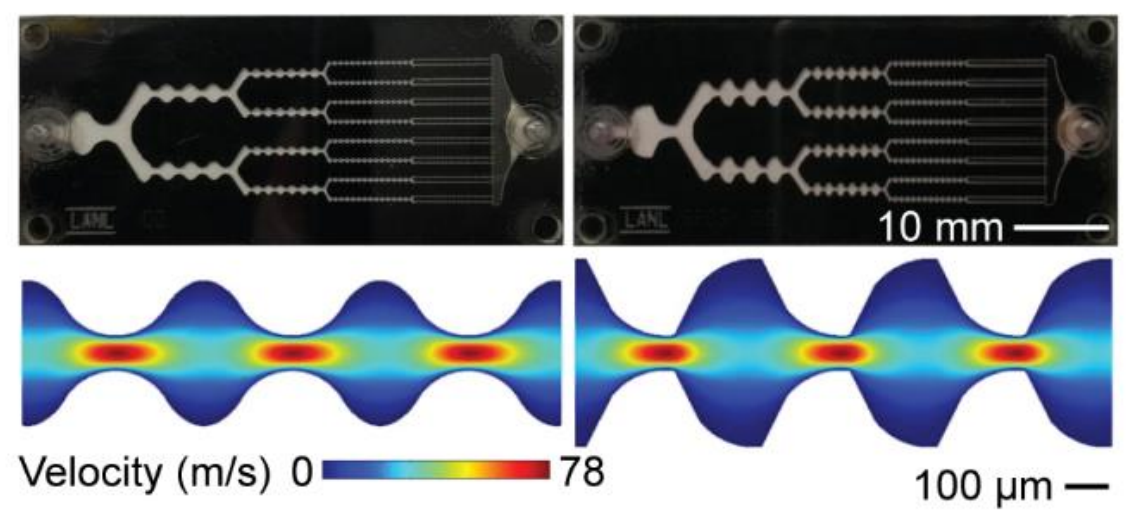

Figure 15. Application of microfluidics to the dissociation and fragmentation of tissues. Hydrodynamic dissociation of tissues into single cells. Original constriction-expansion design (left) and modified shark-fin designs (right) generate high velocity gradients and shear stress environments to dissociate samples. Reproduced from Qiu et al. under a Creative Commons BY 4.0 License. ${ }^{183}$

workflows, tissues are typically dissociated mechanically and/or chemically into single cells, and then analyzed using flow cytometry, sequencing, or other assays. However, conventional mechanical dissociation via vortexing or repeated pipetting is poorly controlled, and chemical dissociation using digestive enzymes must be carefully tuned to minimize the loss of surface markers and avoid excess cell death. ${ }^{184}$

Microfluidic methods offer greater control in generating fluid shear flow environments and thus offer improved mechanical dissociation, which may enable milder enzyme treatments or even enzyme-free dissociation. Lin et al. developed a pillarbased microfluidic cell dissociation chip, which used an array of narrowly spaced microfluidic pillars to dissociate neurospheres into single neural stem/progenitor cells. ${ }^{185}$ The device consisted of an array of rectangular pillars (50 $\mu \mathrm{m}$ wide x 167 $\mu \mathrm{m}$ tall $\mathrm{x} 240 \mu \mathrm{m}$ long). The pillars were arranged in a $142 \mathrm{x}$ 10 array and separated by a gap of $20 \mu \mathrm{m}$ in the lateral direction and a gap of $100 \mu \mathrm{m}$ in the longitudinal direction. Neurospheres were first passed through a $40 \mu \mathrm{m}$ filter and then flowed through the pillar-based device at flow rates ranging from 3 - $15 \mathrm{~mL} / \mathrm{min}$. When operated at $10-15 \mathrm{~mL} / \mathrm{min}$, the maximum shear stress occurred on the sidewall of the micropillar and ranged from $10-18 \mathrm{~Pa}$. The device performance was very favorable, with $90-95 \%$ yield of dissociated single cells from the neurospheres and $80-85 \%$ viability. Additionally, the cells retained their differentiation potential and their ability to regrow into neurospheres. Critically, the high performance of the pillar-based device was achieved without the use of digestive enzymes.

Qiu et al. devised a simple method to dissociate breast cancer tissue samples in a microfluidic device using nylon mesh filters. ${ }^{186}$ The integration of the filter into the microfluidic device was critical to prevent sample loss and clogging at small pore sizes that would occur in bulk conditions. The optimal combination tested was to first pass samples tangential to a $50 \mu \mathrm{m}$ filter and then to pass sample directly through a 15 $\mu \mathrm{m}$ filter. This combination resulted in a single cell yield of $\sim 65 \%$, which was $\sim 3$ times greater than conventional methods, and $\sim 80 \%$ viability. Higher single cell yields could be obtained using smaller filter sizes but came at the cost of drastically reduced viability.
Hydrodynamic forces can also be used to dissociate a tissue sample. Using a series of constriction-expansion channels to generate "hydrodynamic micro-scalpels", Qiu et al. dissociated colon and lung tumor samples into single cells. ${ }^{187}$ The device consisted of a branched design containing 5 stages, each containing a series of constriction-expansion structures (Figure 15). The device channels were laser-cut in polyethylene terephthalate plastic, and the channel height was 300 um throughout the device. The channel layers were sandwiched using adhesives between layers to seal the top and bottom to form the final device. Starting with a single channel of $2 \mathrm{~mm}$ minimum width, the channel number doubled and the channel dimension reduced by half in each stage, ending in a final stage containing 16 channels of $0.125 \mathrm{~mm}$ minimum width. The expansion chambers were roughly square shaped with the diagonal oriented in the flow direction, and a diagonal length three times the constriction width. The constriction-expansion geometries generated regions of high fluid shear in the constriction regions that progressively dissociated the tumor samples, ranging from $\sim 3.5 \mathrm{~Pa}$ in the first stage to $\sim 11 \mathrm{~Pa}$ in the final 5th stage when operated at $12.5 \mathrm{~mL} / \mathrm{min}$. For small cell clusters, a trypsin digest followed by the hydrodynamic microscalpels increased the single cell recovery to $94 \%$ compared with $76 \%$ when using a trypsin digest alone. For intact cell monolayers that were only treated with collagenase to release the monolayers, the total cell recovery rate when operating the device enzyme-free exceeded that of a trypsin digest and repeated pipetting by $30 \%$, and the single cell yield was improved to $95 \%$ from $61 \%$. For tumor spheroids $\sim 200-300 \mu \mathrm{m}$ in diameter, operating the device enzyme-free led to poor results, but a combined enzyme-device treatment greatly increased the total cell recovery by up to 10 -fold compared to enzyme digestion alone, with up to $90 \%$ single cell yield.

The authors further improved this device in a follow-up study by tuning the channel geometry. ${ }^{183}$ They decreased the minimum width to $75 \mu \mathrm{m}$ in the final stage, which increased the maximum shear stress by $150 \%$, and used an extended shark fin geometry in the expansion chambers, which generated an additional large peak in the shear stress along the centerline of the channel (Figure 15). Additionally, the shark-fin device was easier to fabricate and assemble and had improved resolution compared to the original device, since the channels 
were fabricated on a single polyimide film layer using laser micromachining. The optimized shark fin design resulted in a $\sim 20 \%$ increase in single cell yield and a reduction in the fraction of small and large aggregates compared to the original device.

A recent study integrated the hydrodynamic micro-scalpel method and the cell filtration method described above with an on-chip digestion module to form a fully microfluidic platform to process tissues into single cells. ${ }^{188}$ Unlike previous studies described here, this study took the additional step of performing single-cell RNA sequencing on the resulting cell suspension. Using kidney and breast cancer tissue, they found that the fully microfluidic platform yielded $>2$ times more epithelial cells and leukocytes, and $>5$ times more endothelial cells compared with conventional methods. Furthermore, stress response genes were not elevated in samples processed by the microfluidic platform, indicating that the process flow was not excessively harsh on the cells.

While some of these microfluidic methods have demonstrated high performance under enzyme-free conditions, the results are generally further improved using a combination approach of enzyme treatment and microfluidic dissociation. Typically, a combined enzyme and device can achieve similar or even better results in less time compared to conventional methods using the same enzyme treatment.

\subsubsection{Sectioning into small tissue fragments}

Sectioning live tissue into small tissue fragments can be achieved using similar methods to tissue dissociation into single cells. A motivation to generate such fragments is to facilitate rapid cell culture expansion, for example, for clinical use.

Towards this application, Wallman et al. developed Biogrid, a closed and enzyme-free system to dissociate cell aggregates into small fragments. ${ }^{189}$ The Biogrid consisted of multiple rows of thin knives etched into a silicon wafer, aligned parallel to each other in a single direction and covering an area $2.5-$ $6 \mathrm{~mm}^{2}$. The blades were fabricated in silicon using an anisotropic etch, which resulted in vertical sidewalls. Various grid designs ranging from $10-30 \mu \mathrm{m}$ edge thickness and $60-200$ $\mu \mathrm{m}$ spacing were tested. The authors did not report any significant biological differences between the various grid sizes tested. The silicon microgrid was fit into an adaptor inside a fluidic system containing the suspended cell culture. The cell suspension was passed at $0.5-1 \mathrm{~mL} / \mathrm{s}$ across the microgrid to cut large aggregates, without the need for enzymatic treatments or the risk of contamination by manual mincing. Smaller fragment sizes were achieved by passing the sample repeatedly back and forth. The Biogrid was capable of expanding neural stem cell cultures at rates that were comparable to but also more consistent than previous enzymatic methods.

\subsubsection{Bisection}

Studying regeneration in multicellular models such as planarian flatworms could shed insight on the communication and interaction between different parts of the organism. ${ }^{190}$ As is the case in single-cell wound healing, microfluidic devices offer new opportunities and advantages in wounding and studying regeneration in multicellular systems.

For example, bisection of multicellular samples can be achieved in a similar fashion to the bisection of single cells.
Toda et al. adapted the single-cell microfluidic guillotine described in Section 3.1.2 to bisect multicellular structures. ${ }^{191}$ In this study, the authors engineered cells with synthetic surface ligands and receptors that promoted cell-cell adhesion. These engineered cells then self-assembled into three-layered multicellular spheroids. The guillotine geometry was adjusted to $150 \mu \mathrm{m}$ in width and $50 \mu \mathrm{m}$ in height to provide confinement that aligned the spheroid to the blade. The flow velocity was increased to $148 \mathrm{~cm} / \mathrm{s}$, which was much greater than the velocity needed to cut Stentor cells, likely due to the increased stiffness of the spheroid. The organization of these cells was robust and remarkably regenerated its layered structure by 25 hours after bisection.

\subsection{Outlook and future opportunities}

In summary, microfluidic methods can be utilized to perform a diverse set of sectioning surgery, with broad applications across biology. Some of these devices have massively improved the process flow for existing applications, such as for replicative lifespan studies in yeast or for tissue dissociation into single-cells. However, for some applications, especially cell enucleation, the advantages of the microfluidic methods are less clear and require further study. In addition to improving existing applications, other devices such as the microfluidic guillotine and the cancer cell fragmentation obstacles have created new opportunities and enabled studies that could not be realized without microfluidics.

While microfluidic methods have already seen success in cutting some biological materials, stiffer biological materials such as cartilage remain a challenge. A limiting factor in microfluidic sectioning in a pure PDMS system is the ability of the PDMS blade to pierce stiff biological samples, as PDMS is relatively soft (Young's modulus $\sim 2 \mathrm{MPa}$ ) ${ }^{50,51}$. While noncontact hydrodynamic methods may be able to alleviate this issue simply by increasing the flow rate, another solution is to use a stiff material such as silicon (Young's modulus $\sim 140$ $\mathrm{GPa}^{192}$ ) to fabricate the blades, as shown in the Biogrid by Wallman et al. ${ }^{189}$ Several other studies have devised silicon grids with sharp edges that can cut samples into 100-200 $\mu \mathrm{m}$ sized pieces. ${ }^{193,194}$ However, they were not demonstrated on live samples and did not leverage microfluidic methods. An alternative approach is to utilize in situ direct laser writing to fabricate a blade structure made of a stiff photoresist (e.g., the Nanoscribe IP series photoresist has a Young's modulus $\sim 1-4$ $\mathrm{GPa}^{195}$ ) directly inside a microfluidic channel. ${ }^{49}$ This approach also has the advantage of fabricating truly three-dimensional blade structures.

Another limitation in current microfluidic sectioning methods is that it is difficult to control the orientation of the cell or organism and therefore the exact position of the cut. Integration with real-time image analysis and micro-manipulation is likely required to achieve this type of control.

Finally, the current work has revealed gaps in our understanding of the physics of cutting biological tissues. For example, studies on how biological materials are damaged by hydrodynamic forces have been explored for bulk methods such as bioreactors. ${ }^{196}$ However, much less attention has been given to how biological materials are damaged or cut by hydrodynamic forces in microfluidic environments. For single cells, the fluid shear forces on the surface of the cell must introduce sufficiently high membrane tension to create a rupture. The mechanical stress on a single cell arising from fluid forces 


\section{Ablation}
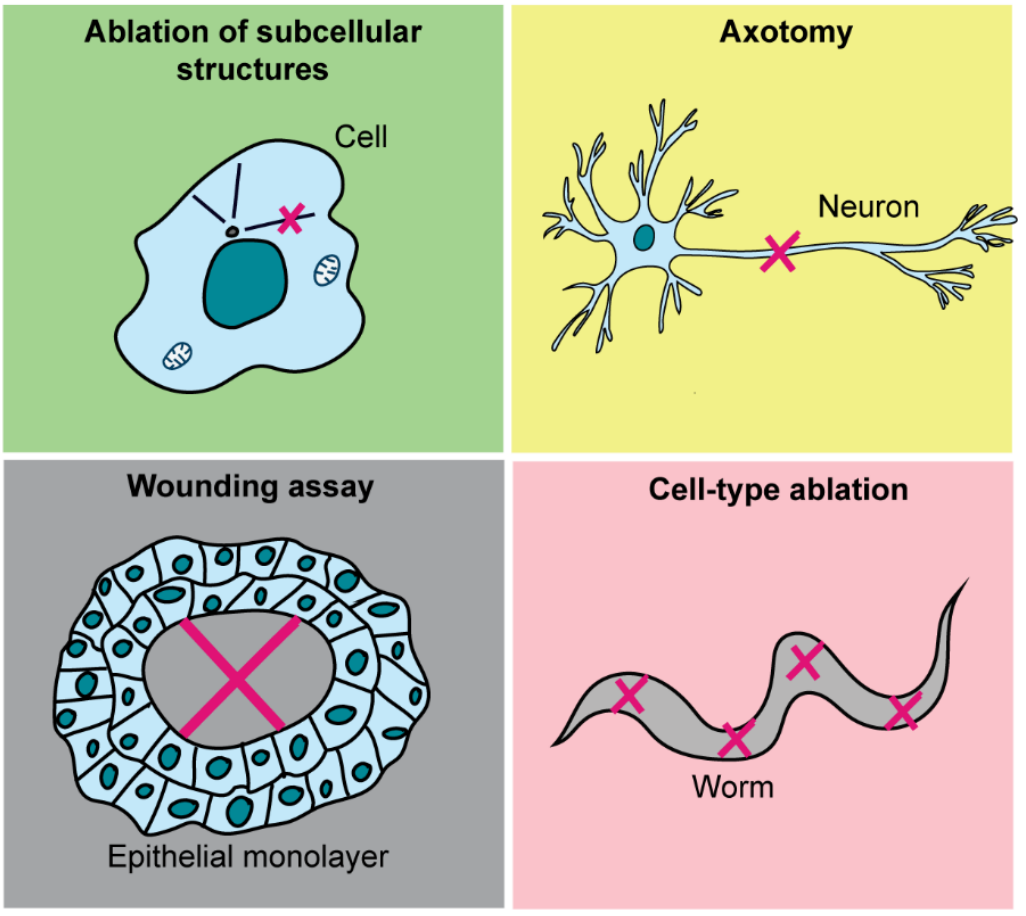

Figure 16. Types of ablation surgery. Four examples of ablation surgery are shown, ablation of subcellular structures (top left), cutting of neuronal axon or axotomy (top right), wounding of an epithelial monolayer (bottom left) and ablation of a specific cell type in a worm (bottom right).

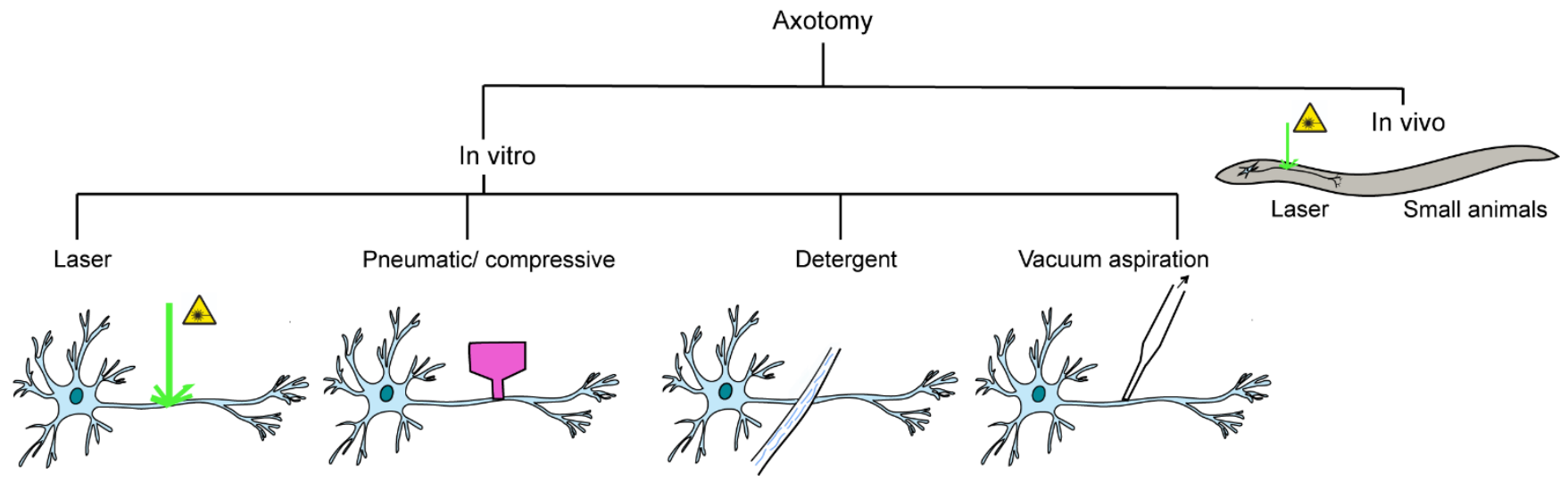

Figure 17. Types of axotomy surgery. Axotomy, or the cutting of an axonal part of a neuron can be carried out either in vitro, at the single cell level, or in intact animals (in vivo). Single neurons can be axotomized by various ways including laser, compression, surfactant flow or vacuum aspiration. Laser is most commonly used for intact animals.

in a microfluidic cross-junction channel has been investigated, although membrane rupture was not considered in this study. ${ }^{197}$ For multicellular tissues, a mixture of all three fracture modes will presumably occur (opening, in-plane shear, and out-of-plane shear; see Section 2.1.1) since the sample orientation is not well-controlled when using hydrodynamic forces, but this process is not well-studied.

\section{ABLATION}

We define ablation as the process of removing or destroying parts of a biological sample. Ablation can involve the cutting of a single cell, for example, a neuron, or deletion of an entire population of cells in an organism (Figure 16). Ablation of subcellular structures, cells and populations of cells within tissues can provide information about the function and mechanical properties of organelles and cells, and their roles in morphogenesis and regeneration. So far, the use of microfluidics for ablation surgeries has predominantly focused on studying the mechanisms of neural regeneration within single neu- 
rons and in intact organisms such worms, flies and zebrafish, as well as in the study of wound healing in cultured cells.

Several advantages of microfluidics have enabled these studies. First, microfluidic devices are transparent. They are thus compatible with ablation using laser light. Cutting procedures can also be visualized as they are performed. Second, laminar flow makes it simple to vary the buffer composition in different micro-compartments, which can reside on top of long cells such as neurons. Streams of fluids can be directed precisely to ablate parts of single or multiple cells. Third, a wide range of microfluidic devices have been developed to capture and immobilize small animals, typically prior to laser ablation. Microfluidic immobilization removes the need for anesthetics, and thus allows for faster regeneration and improves the reproducibility and processing time of ablated cells or animals. Coupled with flow control, ablated samples can be easily flushed out of the microfluidic channel to increase the throughput of surgery.

\subsection{Single cells and subcellular structures}

\subsubsection{Axotomy}

The most widespread use of microfluidics at the single cell level for ablation has been in the axotomies of neurons (Figure 17). Nerve surgery is an ancient discipline dating back to the 7th century AD when Paulus Aeginatus hypothesized that severed nerves could regenerate, but it was Gabriele Ferrara (1543-1627) who described the suturing of nerve stumps in detail. ${ }^{198,199}$ Structural and morphological changes of neurons in response to injury or cutting have been described in seminal works of Nissl (1892) and most notably Ramón y Cajal (1928). ${ }^{200-202}$ These works elucidated biological events following neuronal injury to various parts of the nervous system. In 1975, Grafstein wrote, “Axotomy usually involves removal of a significant portion of the nerve cell volume, but most of the synthetic machinery of the cell, because it is localized in the soma, is left intact, and the defect produced in the surface membrane at the site of amputation is small in relation to the total cell surface that remains". ${ }^{201}$

From these early roots, the use of microfluidics in axotomy started to appear in the 2000s. Readers are also referred to previous literature for comprehensive reviews. ${ }^{203,204}$ For neuron axotomy, microfluidic methods are useful as they allow separate interrogation of different parts of the cell. A common approach used in several studies is the spatial separation of the cell body and axon in different compartments connected by tiny microchannels through which the axon traverses (Figure $18 \mathrm{~A}, \mathrm{~B}){ }^{205}$ These ex vivo studies have allowed a greater variety of axotomy methods, as detailed below, than in vivo studies.

\subsubsection{Axotomy by vacuum aspiration}

Axotomy by vacuum is a common technique to injure axons. The first study demonstrating this technique grew cortical and hippocampal rat and mouse neurons in 2-compartment devices. ${ }^{206}$ Axons traversed through $10 \mu \mathrm{m}$ wide microgrooves, away from the somal compartment, eventually reaching the second compartment of the device (Figure 18A). Minute volume differences between compartments and the high fluidic resistance of the microgrooves created a flow that counteracted diffusion of chemicals between the somal and axonal compartments. Axons were injured simply by applying vacuum aspiration to the axonal compartment. An example from a different study that also used vacuum aspiration is shown. ${ }^{207}$ Soma were then collected to assay resultant transcriptional changes.

Fluidic isolation was exploited to locally apply neutrophins in the axonal compartment to study their effects on regeneration. Several subsequent studies have used similar approach based on aspiration to injure cultured neuronal cells. ${ }^{207-213}$
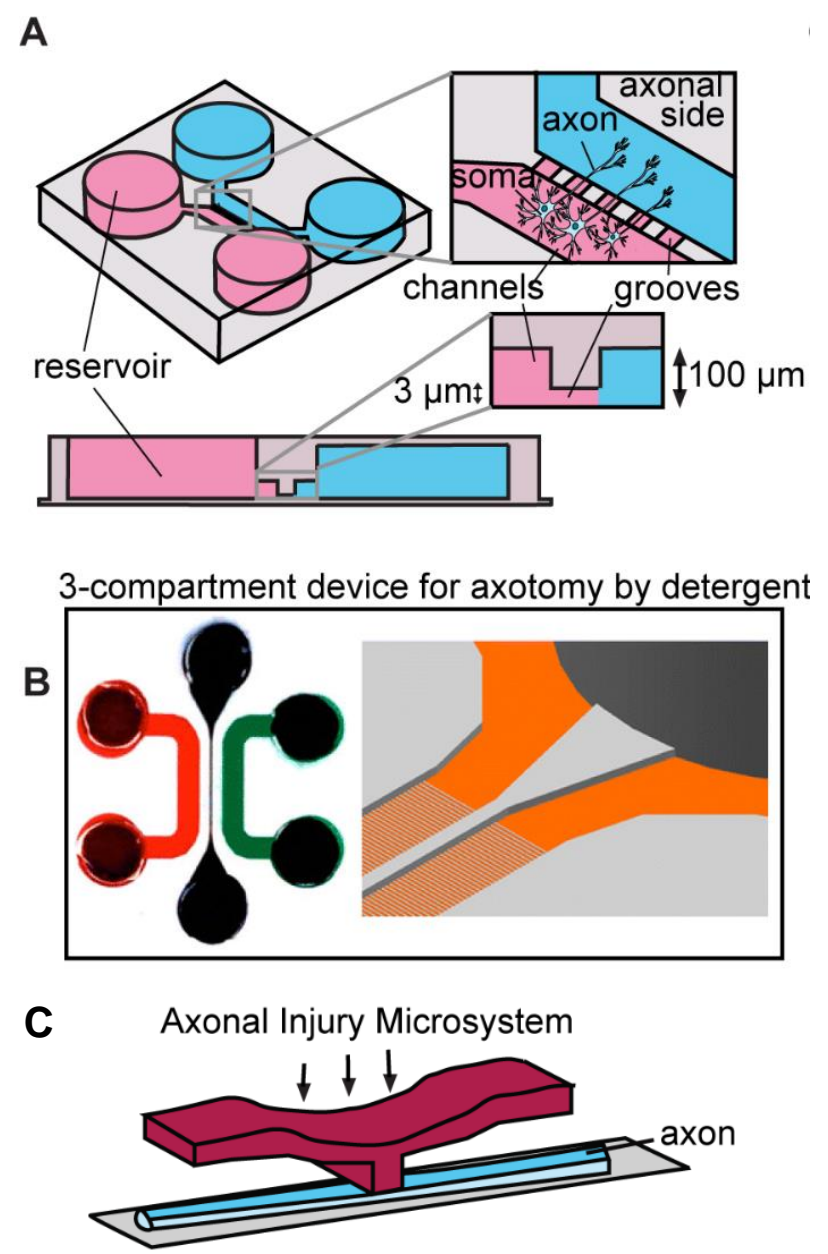

Axotomy by compression

Figure 18. Devices and techniques used in single neuron axotomy. (A) 2-compartment device used in neuronal culturing to spatially separate soma and axons, connected by minute microgrooves. (B) 3-compartment device used to carry out axotomy by detergent. Distinct compartments are indicated by the colors. Detail of the microgrooves is shown in the right panel. Reproduced from Kilinc et al. under a Creative Commons BY-NC 2.0 license. ${ }^{214}$ (C) Schematic of valve-based, $\mathrm{CO}_{2}$ routing, axonal injury microsystem used to carry out axotomy by compression.

\subsubsection{Axotomy by introduction of surfactants}

Kilinc et al. designed a 3-compartment device using a principle similar to the 2-compartment device described above (Figure 18B). ${ }^{214}$ The third compartment was centered on top of 
the axons. Axotomy was carried out on primary mouse neural cultures by flowing $0.1 \%$ saponin solution through the central (third) compartment between the somatic and axonal compartments. The other compartments were held at high pressure to localize detergent flow. This device was used to demonstrate degeneration of the distal part of the axon that was not connected to the soma after axotomy. Other surfactants such as sodium dodecyl sulfate (SDS) and Triton-X100 have also been used in similar studies. ${ }^{215,216,217}$

\subsubsection{Axotomy by compression}

Microfluidic devices have also been used to apply mechanical compression to injure axons and study their repair process. These experiments may serve to mimic traumatic brain injury or spinal cord injuries. ${ }^{204}$ Hosmane et al. devised a method to apply compressive injury on axons to mimic traumatic axonal injuries (Figure 18C). ${ }^{218}$ The 2-layered device contained 3 compartments, for the isolation of cell bodies, proximal axons, and distal axons, respectively. The second layer was a valvebased elastomeric injury pad for inflicting micron-scale mechanical injuries on the axons. This setup was used to injure primary hippocampal neurons from rat pups by lowering the injury pads, which were actuated by increasing the pressure of the gas applied to the second layer. Unlike previous devices based on vacuum aspiration and surfactant, this device allowed simple control of the degree of injury to the axons. By increasing the pressure applied to the second layer, the injury pads were lowered to a greater extent to increase the compression on the axons. The compression was held for approximately 5 seconds. The axons displayed a graded response to the injury. After a small, transient compression from the injury pad, axons remained healthy and continued to grow. But after a large, transient compression, complete transection or rapid severing of axons was observed.

\subsubsection{Axotomy by laser ablation}

By far, laser ablation is the most versatile tool for ablation (also see details in Section 2.1.2). ${ }^{219,220}$ It can be used not only to ablate single neurons in vitro inside microfluidic devices similar to those described above, but also to ablate neurons in vivo inside intact animals (see Section 4.3). The optical transparency of PDMS and glass substrates from $\sim 400 \mathrm{~nm}$ to 1000 $\mathrm{nm}$ facilitated their use in laser ablation experiments. ${ }^{73}$ At the single cell level, femtosecond laser ablation has been used to sever axons and minimize thermal damage beyond the ablation zone. ${ }^{66}$ Dorsal root ganglion and cortical neurons were isolated from embryonic rat pups and grown in the classic 2compartment microfluidic isolation device for neural studies. In this study, post-axotomy visualization was difficult due to the immediate recoil of axons when a certain energy deposition threshold was exceeded. However, the regeneration of axotomized neurons was successfully observed. Targeted ablation of axons was also achieved on micropatterned PDMS substrates, and regeneration was observed in the presence and absence of calcium, after partial and complete transection of axons by laser. ${ }^{221}$

\subsubsection{Microtubule lattice ablation}

Microfluidics has been used to study the nature of dynamic instability of microtubules in vitro by inducing damage to the microtubule lattice and observing its self-repair. ${ }^{222}$ Here, mi- crotubules were assembled inside a microfluidic chamber and focused laser light was used to induce photodamage onto the microtubule lattice. The laser power used was above that needed for bleaching but below the severing threshold, and was shown to be capable of inducing local damage and to promote self-repair of microtubules. ${ }^{223,224}$ Microfluidic flow was used to introduce free tubulin monomer that would elongate filament "seeds" to form long filaments, and was used to flush out unpolymerized monomer after a certain elongation time. This work showed that free tubulin can be incorporated not only at the ends but along the length (sides) of microtubules, especially in areas where they experience physical constraints such as bending. This study also illustrated the flexibility offered by microfluidic systems in switching out solutions and carrying out perturbations without disrupting the system (in this case, microtubules) being studied.

\subsection{In vitro cultured cells}

Another example of use of ablation surgery is the classic wound healing assay. Wound healing involves multiple biological processes working in concert, for example, cell migration, adhesion and division, and is richly informative when induced in vitro. A common method to study wound healing at the multicellular level is the scratch wound assay. In the traditional version of the scratch assay, a wound is manually generated in an adherent cell culture, often by scratching with a pipette. The subsequent migration, proliferation and other behaviors of the cells to fill the scratched or wounded area are then observed. The scratch wound assay can thus be considered a form of ablation surgery, in which a region of the monolayer of cells is removed. Scratch assays were carried out as early as 1965 when Todaro et al. induced a wound in a monolayer of contact-inhibited 3T3 cells cells, to see if the wound stimulus would reactivate cell division. ${ }^{225}$ This process was also repeated with chick embryo fibroblasts. ${ }^{226}$

Microfluidic methods can make the scratch wound assay more controllable and repeatable than manual scratching (Figure 19). They also allow for constant infusion of nutrients and waste removal. Other advantages include less injury to the cells, retention of the extracellular matrix, removal of cell debris, and automation. For detailed discussion on cutaneous wound healing studies, the readers are referred to a prior review. ${ }^{227}$

\subsubsection{Wounding by compression}

Sticker et al. used a pneumatically operated device to study the wound healing of HUVECs (Figure 19A). The device contained a bottom microfluidic channel layer, and a top pneumatic layer separated by a flexible PDMS membrane. ${ }^{228}$ When the PDMS membrane was pushed down, the cells underneath were removed from the rest of the cell layer and destroyed. The ensuing cell migration into the "wound" was observed. By analyzing the fluorescence of fibrinogen remaining at the wound site, the study confirmed that the extracellular matrix (ECM) was retained in the pneumatic device, but not when the monolayer was physically scratched with a stylus. There was also minimal death of cells at the edge of the wounds in the microfluidic assay.

A similar pneumatically-controlled membrane-wounding method was employed by Go and colleagues. ${ }^{229}$ Some wound healing studies have used geometrical constraints to seed cells 
around an empty region, followed by a release of those constraints to observe cell migration into that region. For example, Zhang et al. fabricated a two-layered device where cells grew around removable PDMS pillars. ${ }^{230-233}$ Wang et al. devised a system to circumvent the limitations of $2 \mathrm{D}$ cell culture systems using an SU-8 mesh chip that would have a 3D aggregate of cells with a hole in the middle. ${ }^{234}$.

\subsubsection{Wounding by introduction of chemicals}

In addition to the mechanical approach described above, microfluidic implementation of the scratch wound assay can also be achieved chemically. A laminar co-flow design allows the flow of tightly controlled streams of reagents over cells cultured inside a microfluidic device. Typically, a stream of trypsin, lysate, or drug is introduced in the center of the channel to generate a wounded area. This stream is sandwiched by
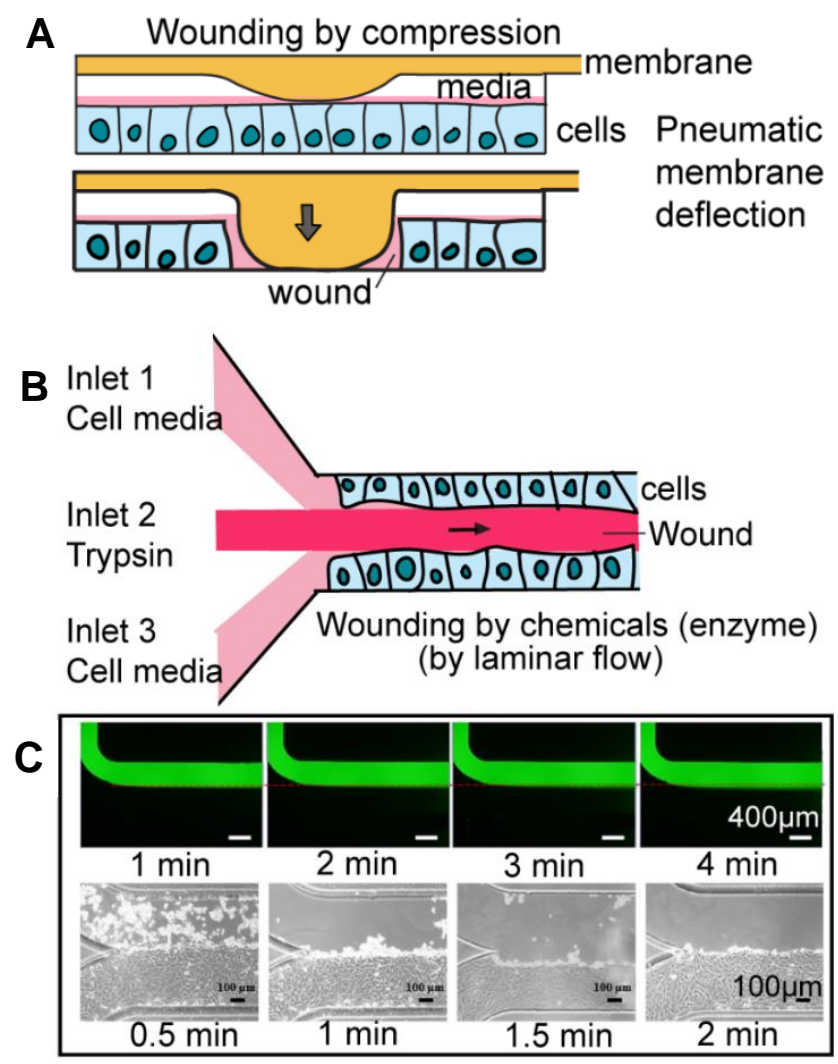

Figure 19. Types of devices and techniques used in the microfluidic generation of wounds in cell monolayers. (A) Sketch of a pneumatically controlled device that generates wounds in a HUVEC monolayer by compression. (B) Cartoon of a device to generate wounds in a cell monolayer in a microfluidic device with 3 separate inlets converging into a single channel is shown. Trypsin is injected through the middle channel, and a wound is caused due to laminar flow. (C) Pump-free, gravity-driven two-inlet device, where the height difference between the inlet and outlet channels drives the fluid-flow. Laminar flow is maintained over a period of many minutes (green- FITC dextran, upper panels). Lower panel shows that 2 minutes of exposure to trypsin is sufficient to generate a wound in a monolayer of T/G HA-VSMCs (aortic vascular smooth muscle cells). Reproduced from Wei et al. under a Creative Commons BY 4.0 License. ${ }^{235}$ two streams containing cell culture media for the unwounded areas (Figure 19B,C).

These studies recapitulated findings from traditional scratch wound assays, but offered higher precision and thus enabled systematic exploration of parameters such as wound size. Additionally, a microfluidic scratch wound assay can explore more complex experimental parameters such as the effect of fluid shear stress. Several studies have utilized laminar flows of trypsin, a protease that detaches cells, to wound monolayers chemically. ${ }^{235-241}$ Laminar streams of trypsin have also been used in combination with nanopatterning to study the influence of ECM topography on wound healing. ${ }^{242}$

\subsubsection{Wounding by application of hydrodynamic shear} force

Shear force has been used to detach cells and create wounds. Soitu et al. used microjetting to create wounds on confluent monolayers. ${ }^{243}$ An immiscible, biocompatible fluorocarbon oil (FC-40) was poured over cells in a dish with media. A jet of FC-40 was introduced over the cells and the resulting fluid flow resulted in cell detachment. FC-40 flow rates above $5.4 \mu \mathrm{L} / \mathrm{s}$ produced wounds. Different types of wound thickness, size and shapes were generated by changing the jetting parameters such as flow rate and changing the position of the needle over the monolayer.

\subsection{Multicellular organisms}

Precision cuts are critical for in vivo surgeries in live multicellular organisms. Microfluidic surgery in multicellular organisms have thus far been performed exclusively using laser ablation. The key role of microfluidics is the immobilization of the organism. Microfluidics also enables the automation of the handling of the organisms and allows for high throughput surgeries to generate enough samples for subsequent studies that require a large number of specimens, such as RNA sequencing or population behavior assays. For additional reviews on animal microsurgery, we refer the readers to prior literature. ${ }^{55,220,244}$ Below, we will review the microfluidic methods used for immobilization of live organisms for laser ablation studies.

\subsubsection{Immobilization by applying pressure}

Immobilization by the application of pressure on living organisms has been carried out alone or in combination with the application of carbon dioxide to the organisms. Guo et al. designed an adjustable microfluidic trap to ablate axons in live C. elegans using a femtosecond laser. ${ }^{61}$ The device was 2 layered. Worms were loaded in the bottom layer, with a thin membrane separating it from the top layer which was pneumatically controlled. Pressurization of the top layer deflected the membrane and immobilized the worm (Figure 20A). It also flattened the animal against the cover glass on the bottom of the channel, thereby allowing the axon to be in focus for imaging and ablation. Survival tests carried out on 20 worms immobilized at $110 \mathrm{kPa}$ for 60 minutes showed no defects or death. This work demonstrated that immobilization by microfluidics was effective and removed the need for anesthetics, which delayed regeneration by several hours. Gokce et al. used a similar approach for fully-automated ablation in $C$. 

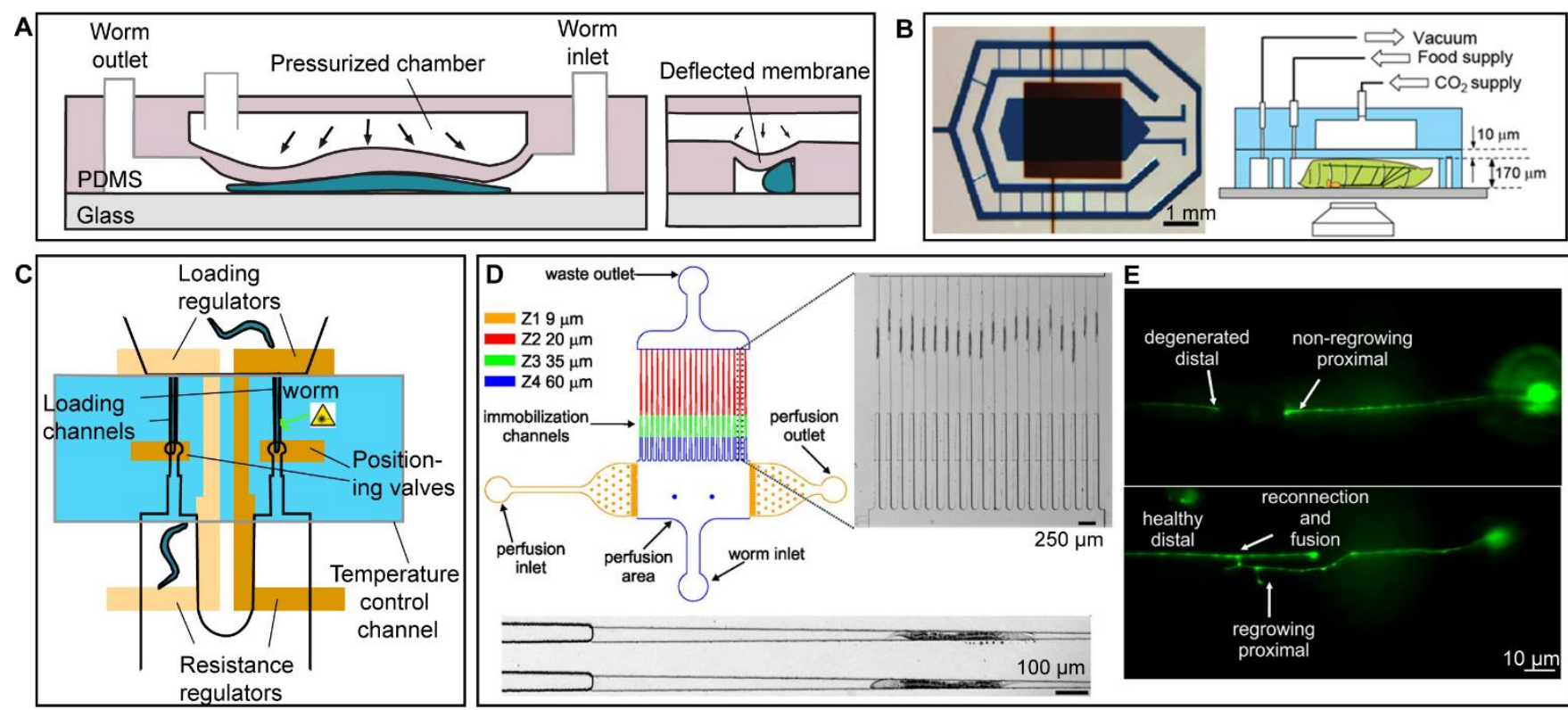

Figure 20. Microfluidic devices for small animal immobilization and surgery. (A) Cartoon of a pressure-based immobilization device for C. elegans that uses a deformable PDMS membrane to hold the animal still. ${ }^{244}$ (B) 2-layer, long immobilization device for Drosophila. The first layer (blue) provides food to the animal, whereas the second layer (red) supplies $\mathrm{CO}_{2}$ for immobilization through a $10 \mu \mathrm{m}$ PDMS membrane (left panel), as depicted by the adjoining cartoon (right panel). Copyright @ 2012 Ghannad-Rezaie et al. and reproduced under a Creative Commons Attribution license. ${ }^{68}$ (C) Cartoon of temperature-controlled immobilization device for automated killing of cells in $C$. elegans. Valves in light yellow are open, whereas valves in dark yellow are partially closed. (D) Worm hospital device (left panel) that uses a set of tapering channels/ passive clamps (right panel, magnified view: bottom panel) to immobilize L4 worms at a press ure of 65 $\mathrm{kPa}$. (E) Worms containing the transgene $z d l s 5$ to label the neurons with GFP. Axotomized PLM neuron from a worm having a mutation in the WNT ligand EGL-20 showing no regeneration (top panel) and PLM neuron from a zdls5 (control) worm showing successful reconnection (bottom panel). Reproduced from Gokce et al. under a Creative Commons BY 4.0 license. ${ }^{65}$

elegans. Individual axotomies at a rate of roughly $17 \mathrm{~s} /$ worm was achieved. ${ }^{64}$

Immobilization of $C$. elegans using a combination of aspiration and a pressurized membrane was demonstrated along with a high throughput screen of compounds that increased neuronal regeneration. ${ }^{63,245,246}$ Thousands of animals were screened using an automatic system that transferred the animals from multiwell plates into a microfluidic device that used a single aspiration port to immobilize a single animal at a time with a pressurized membrane system for fast axotomy. The single aspiration port allowed the capture of a single animal in a screening chamber, while a pressure pulse was applied to move debris and other animals away from the chamber. Then, all valves were closed and the animal was moved towards a channel array. The membrane above the chamber was then deformed to tightly immobilize the animal.

Chokshi et al. used the 2-layered design along with carbon dioxide perfusion through the thin membrane to increase immobilization times to several hours. ${ }^{247}$ A similar strategy was used to immobilize Drosophila 3rd instar larvae either by applying pressure on the membrane alone for $\sim 1$ hour in a snug microchamber or several hours in combination with $\mathrm{CO}_{2}$ (Figure 20B). ${ }^{68}$ This device was used to detect intracellular calcium waves and axonal transport patterns in proximal (to the cell body) and distal fragments in class IV sensory neurons. This study also confirmed that animals did not require any post-operative recovery time when immobilized using microfluidics, as opposed to those that are immobilized using anes- thetics like isofluorane, which required 2 hours between imaging sessions.

\subsubsection{Immobilization by cooling}

Chung and Lu designed a completely automatic system for ablating the olfactory neurons AWBL/R that expressed a GFP 7-transmembrane receptor in worms. ${ }^{62}$ In this study, a specific population of cells were "killed" and no fine surgery on axons was carried out (Figure 20C). The device contained two worm loading channels on each side, with a loading and resistance regulator. It had a unique mechanism to only load a single worm at a time, as the presence of a worm in the loading channel increased its resistance and caused the worms to be diverted into the empty channel. Once the worm entered a channel, the animals were immobilized by transient on-chip cooling. Cooling stopped body and pharyngeal movements but did not change the size or orientation of worms. Automatic imaging and thresholding of GFP-expressing neurons was used to locate cells to be ablated. Using this setup, the authors were able to ablate 350 pairs of olfactory neurons assaying success using a chemical repellent assay. A throughput of 110 worms/hour was achieved, sufficient for a population behavior assay.

\subsubsection{Passive immobilization}

Passive immobilization of $C$. elegans has been carried out by using a series of clamps, first described by Hulme et al., which consisted of a network of tapered channels that gradual- 
ly immobilized the animals. ${ }^{248}$ By choosing to operate the device at a constant pressure, rather than a constant flow, the authors were able to avoid mechanical damage to the animal. One of the key advantages of the immobilization device was that it contained bifurcation channels, as opposed to trifurcations or higher order branching topologies. This design facilitated the addition of bifurcation points to increase the number of clamps $\left(2^{\mathrm{N}}\right)$ and ensured identical fluidic resistance of each branch. Pinan-Lucarre et al. used this design to discover a beneficial role of CED-3 caspase in preserving dissociated processes of ablated ALM mechanosensory neurons. ${ }^{249}$

Allen et al. also used this system of tapered microchannels to study synaptogenesis in the HSNL neuron. ${ }^{250}$ The study showed that ablating the synapse at a crucial point in its development led to ectopic synapse formation.

Gokce et al. modified the design of passive clamps to reduce the height and the width to prevent the rotation of the lateral axis of animals away from the image plane. ${ }^{65}$ The height was reduced in 3 steps along with the tapering width to maintain an aspect ratio of $\sim 1$ at each step. This system was used to investigate the role of WNT/Frizzled on axon regeneration by cutting ALM and PLM neurons in C. elegans (Figure 20D,E). The trapping efficiency of this setup was over $91 \%$ with or without anesthetics. In order to perform long-term regeneration studies of the entire nerve, this device included an imaging module and a post-surgery module for 24-hour recovery. There were sieve channels on either side of the perfusion area that connected the inlet to the outlet. The perfusion inlet provided food for the axotomized animals. After 24 hours of recovery, the worms could be retrapped and imaged at efficiencies over $83 \%$. The perfusion arms were designed asymmetrically, i.e., the inlet and outlet arms had different lengths to achieve different levels of hydraulic resistance. As a result, the outlet arm was at a low pressure and the animals could be removed from immobilization channels.

In another study utilizing passive immobilization, Chang et al. created a fully automated system that aspirated zebrafish larvae from multiwell plates into a borosilicate capillary. ${ }^{251}$ Although it was not a traditional PDMS-based device, the authors utilized a microfluidic setup to carry out animal microsurgery. By detecting the amount of light scattered/transmitted, the system automatically detected whether the aspirated sample was larvae or debris. The borosilicate capillary with an ultrathin wall $(10 \mu \mathrm{m})$ was found to achieve the best quality in high-resolution confocal imaging with the least amount of autofluorescence compared with capillaries made of other materials such as teflon and thicker capillary walls. To reduce mismatch in refractive index and optical distortion, the capillary was immersed in water. This system allowed the control of the orientation of the larvae as the animal could be positioned and rotated in the capillary. Positioning and rotation were carried out by determining the entry and lateral orientations using 2D imaging while rotating the capillary and comparing the image to a series of templates. This system was used to carry out axotomy of the lateral-neuron axon fiber bundle in larvae. ${ }^{252}$ Each animal was positioned and axotomized within 18 seconds, with $100 \%$ recovery post surgery.

\subsection{Outlook and future opportunities}

Axotomy and wound healing assays are only two examples of ablation surgeries that have been carried out rapidly and reproducibly with the aid of microfluidics. The same principle should also apply to other types of tissues such as muscle or epithelial cells.

In addition to the techniques discussed above for wounding cells, other methods have been used to remove cells from culture substrates. These methods can, in principle, also be applied to study wound healing in a microfluidic system.

For example, temperature control has been used to detach cells from a Petri dish uniformly coated with the thermoresponsive polymer poly( $\mathrm{N}$-isopropylacrylamide) (PIPAAm). PIPAAm is hydrated and extended below $32^{\circ} \mathrm{C}$ but contracts above this temperature. By lowering the temperature from $37^{\circ} \mathrm{C}$ to $4-10^{\circ} \mathrm{C}$, cell detachment occurred and large gaps in the cultured monolayer were created. ${ }^{253,254}$ Building on this concept, a photoresponsive culture surface was developed using a novel polymer pNSp-NIPAAm, which was a pNIPAAm-based copolymer containing a photoresponsive nitrospiropyran (NSp) component. ${ }^{255}$ UV light was used to increase the polymer's adhesive properties by promoting photoisomerization of NSp. UV irradiation prevented lowtemperature cell detachment whereas irradiation with visible light reversed the effect of UV light. As another example,

focused sound waves have been used to detach cells from a substrate in a non-contact manner. Areas of roughly 200 cells $\left(0.1 \mathrm{~mm}^{2}\right)$ were cleared using an ultrasonic horn and as low as 10 -cell areas were cleared using a focused surface acoustic wave. 256,257

Integrating and automating immobilization, imaging and machine learning with laser ablation may well herald an era where we can carry out ablation experiments and regeneration assays in cells and animals with just the push of a button. Achieving such a goal would require further improvements on immobilization methods since cuts in tissues such as muscles might induce animal movement. Additional innovations in immobilization methods may also be needed for organisms that are soft but motile and extremely responsive to stimuli such as the ciliate Stentor coeruleus. ${ }^{6,7}$ Finally, the combination of genetically-encoded phototoxic proteins ${ }^{258}$ with laser irradiation could further expand control over the types and the precision of cells and structures to be ablated. 


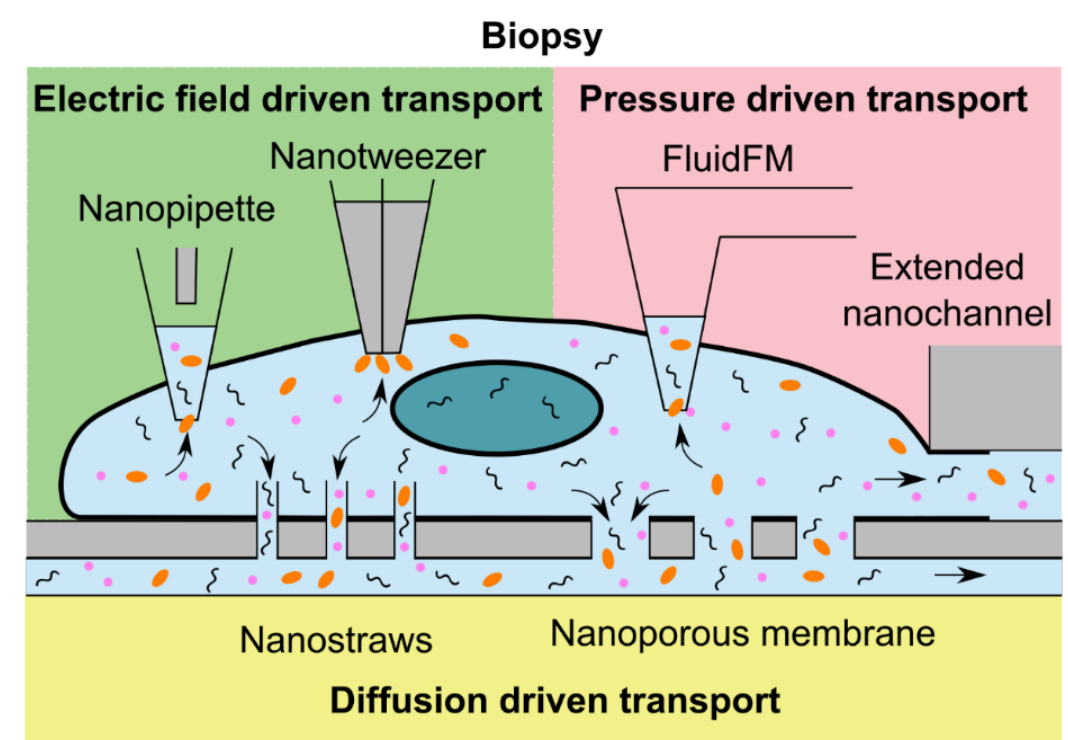

Figure 21. Methods for performing microfluidic biopsy of a single cell. Different techniques for performing biopsy on a single cell are categorized based on their principles of operation: Electric field driven transport (nanopipettes and nanotweezers), pressure driven transport (fluidic force microscopy and extended nanochannels), and diffusion driven transport (nanostraws and nanoporous membranes).

\section{BIOPSY}

We define biopsy as the process of extracting materials from within a living cell or organism for further examination. With advances in imaging, microscale and nanoscale manipulation techniques, biopsy has become increasingly precise and has made possible the analysis of the contents of a living cell. Single cell biopsy has multiple applications in cell biology and medicine. For example, real time monitoring of essential cellular processes such as cell cycle regulation could help shed light on the functions of specific biomolecules. ${ }^{259}$ Real time genomic analysis of cellular contents enabled by biopsy could facilitate the monitoring of disease phenotypes, a prime example being cancer cell transformation, proliferation and metastasis. $^{260}$

Conventional methods for the extraction of proteins and nucleic acids from the cell involve cell lysis. ${ }^{261-263}$ Intracellular fluorescence techniques, such as those based on fluorescent proteins, have allowed time-resolved, longitudinal monitoring of the same population of live cells to some extent. ${ }^{264}$ However, fluorescence methods have drawbacks, ${ }^{265}$ such as the loss of fluorescence due to photobleaching, cell division or other processes, ${ }^{266}$ and potential cytotoxicity and disruption to the normal functions of the cells. ${ }^{267,268}$

Biopsy of cellular contents from live cells can remedy some of these challenges. To extract biomolecules from live cells, micropipettes, also referred to as microcapillaries, have been used for single cell biopsy. Early micropipette biopsy involved the enucleation of sea urchin eggs. ${ }^{269}$ Micropipettes have also been used to extract organelles such as mitochondria, ${ }^{270}$ and to isolate biomolecules such as mRNA $^{271,272}$ and proteins. ${ }^{272,273}$ Biopsy using micropipettes is typically achieved by microscale pressure driven flow. ${ }^{271,272}$ However, cell sampling using micropipettes is limited by a number of factors. Due to the relatively large tip diameter $(\sim 0.5-10 \mu \mathrm{m})$, micropipettes are limited to handling large cells. ${ }^{263,274,275}$ Micropipette biopsy of cells smaller than $20 \mu \mathrm{m}$ suffers from low cell viability. ${ }^{270} \mathrm{It}$ is also difficult to sample precisely from a target location within the cell using micropippettes. ${ }^{270}$

With advances in micro and nanofabrication methods, it is now possible to perform biopsy in a minimally invasive manner to preserve cell viability in a broad range of single cells. For example, atomic force microscopy (AFM) tips and nanoneedles, often functionalized with special surface chemistries, have been used for extracting specific cellular materials. ${ }^{276-278}$ These techniques improved the specificity of intracellular sampling and preserved cell viability, making it feasible for real time monitoring of cells. Recently, the combination of nanoscale devices with micro and nanofluidics has further enhanced the throughput and efficiency of single cell biopsy. In this section, we will review these recent developments for single cell biopsy using nanopipettes or other conduits with diameters in the nanoscale (Figure 21).

We group these methods and related devices based on the way the flow was driven: electric field, diffusion, and pressure driven flows, respectively. Since the fabrication of these biopsy devices are different from those of standard microfluidic devices, we will also summarize the fabrication techniques briefly.

\subsection{Transport driven by electric field}

\subsubsection{Nanopipettes}

Nanopipettes are nanoscale hollow needles typically having a pore diameter of $200 \mathrm{~nm}$ or less. ${ }^{279-282}$ The small size makes them ideal for piercing the plasma membrane and extracting cellular content out of the cells. Nanopipettes described so far operate on the principles of electroosmosis, electrophoresis, and electrowetting to transport molecules and/or organelles out of the cell. The transport is non-specific. Molecules or organelles smaller than the pore diameter could be extracted out of the cytoplasm. 
A

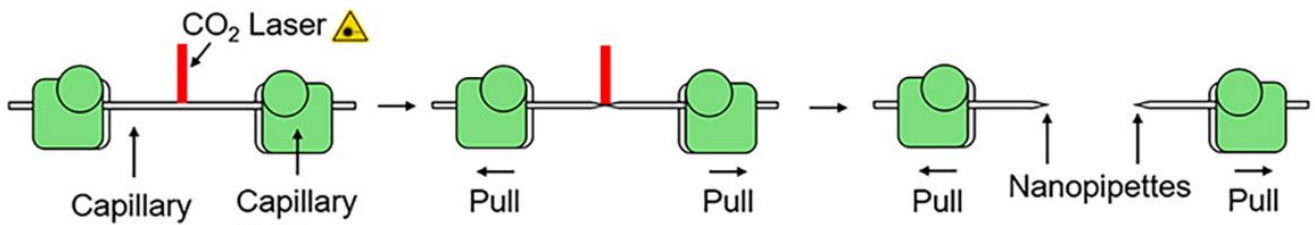

B

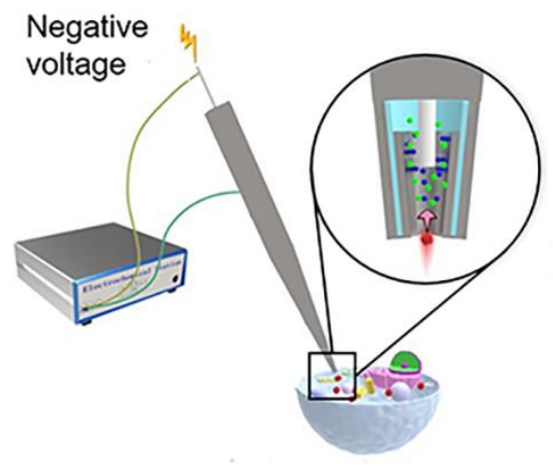

Figure 22. Biopsy using nanopipettes. (A) Schematic diagram showing the fabrication of a nanopipette using a laser puller. Adapted and redrawn from Gao et al. ${ }^{283}$ (B) Schematic of the experimental set-up for performing biopsy using a nanopipette. A negative voltage is applied across the nanopipette using electrodes to extract cellular samples. Reproduced from Pan et al. under a Creative Commons BY-NCND 4.0 license. ${ }^{284}$

Most nanopipettes have been fabricated out of quartz or borosilicate glass capillaries. Nanopipettes are typically fabricated by a laser based pipette puller, where glass capillaries were subjected to alternate cycles of heating by laser irradiation and pulling to generate fine tips of desired diameters (Figure 22A). ${ }^{279,285-287}$ Borosilicate capillaries are softer than quartz, and have been used to fabricate nanopipettes with diameters larger than $80 \mathrm{~nm}$, while quartz capillaries for finer tips as small as $10 \mathrm{~nm}$ in diameter. ${ }^{279}$

Based on the principle of molecule transport, nanopipettes can be classified broadly into two categories. The first category of nanopipettes utilize a combination of electroosmotic flows and electrophoresis to extract cellular content (or to deliver cargo into) a cell. ${ }^{287-290}$ To extract cellular contents, the nanopipette is first filled with an electrolyte solution (e.g. ammonium acetate solution). ${ }^{287,290}$ An electrode is then inserted into the nanopipette and immersed into the electrolyte solution. The counter electrode is inserted in the culture medium. The schematic diagram of a typical experimental set-up is shown in Figure 22B. When a voltage is applied across the nanopipette, an electroosmotic flow is generated. Depending on the dimensions of the nanopipette and the experimental setup, voltages ranging from $2 \mathrm{~V}^{287}$ to $150 \mathrm{~V}^{290}$ have been applied for intracellular sampling. A negative bias draws cytosolic materials into the nanopipette, while a positive bias delivers liquids from the nanopipette into the cell. Transport of molecules in the nanopipette is driven by a combination of the electroosmotic flow and the electrophoretic forces. ${ }^{289}$ The rate of transport was determined by the applied bias, zeta potential of the electrolyte, the size and the electrical properties of the molecules being transported. Cytosolic volume ranging from 2 $\mathrm{pL}$ to $3.5 \mathrm{~nL}$ has been extracted from a single cell by electroosmosis using nanopipettes. ${ }^{287,290}$

The second category of nanopipettes operated on the principle of electrowetting. ${ }^{260,291}$ Samples with volumes from attoliters to picoliters were transferred by changing the surface ten- sion between two immiscible fluids upon the application of an electric field inside the nanopipette. The nanopipette was typically filled with an organic solution such as 1,2dichloroethane. It was then immersed into an aqueous solution (e.g., inside a cell), forming a liquid-liquid interface at or close to the nanopipette opening. An electrode was immersed into the filled nanopipette and a counter electrode was placed inside the outer solution, usually the culture medium. A negative voltage (with a magnitude of 100 s of $\mathrm{mV}$ ) applied across this interface induced a change in the interfacial tension, and caused the aqueous solution to flow into the nanopipette. The electrokinetic processes of electroosmosis and electrophoresis were slower than the time scale over which a change in surface tension occurred. The extraction of molecules in the electrowetting-based nanopipette was thus dominated by surface tension.

In all nanopipettes described above, the volume aspirated by nanopipettes depended on the dimensions of the tip, and the amplitude and the operating time of the applied voltage. The concentration and the properties of the electrolyte in the fluid media are also important operating parameters.

A wide range of molecules have been extracted using nanopipettes. For example, Yin et al. demonstrated the extraction and mass spectrometric analysis of glucose in live epidermal cells from the bulb of Allium cepa (yellow onion). ${ }^{287}$ Chen et al. used nanopipette to extract from zebrafish embryos to characterize yolk cholesterol concentrations at different time points. ${ }^{290}$ Various studies have used nanopipettes to extract and analyze mRNA from HeLa cells, ${ }^{260}$ human MCF-7 cells, ${ }^{292,293}$ mouse embryonic stem cells and 3T3 fibroblasts. ${ }^{292}$ In a recent study, Wang et al. analyzed the levels of miR-10b in MCF-10A, MCF-7 and MDA-MB-231 cells. ${ }^{294}$ Coupled with a high precision of positioning of the nanopipette, spatial localization of specific mRNA has also been demonstrated. ${ }^{292}$ Nanopipettes have also been used to extract subcellular organelles such as mitochondria. ${ }^{295}$ Actis et al. used a $100 \mathrm{~nm}$ na- 
nopipette to extract mitochondria from human BJ fibroblast cells. ${ }^{260}$ Pan et al. extracted lysosomes from a cell using a 130 $\mathrm{nm}$ nanopipette. ${ }^{284}$ The activity of beta glucosidase, a protein specific to lysosomes, was analyzed inside the nanopipette with embedded electrochemical function.

Extraction of cellular materials can cause disruption to normal cell function. However, since the size of the puncture is small, and the volume of sample extracted $(\sim 50 \mathrm{fL}$ or $1 \%$ of cell volume) is minute, the cell viability post aspiration is reasonably high (over $70 \%$ cells survive). The same cell can thus be sampled multiple times at different phases of the cell cycle. $^{260}$

\subsubsection{Dielectrophoretic nanotweezer}

A nanotweezer was developed to trap molecules at the tip of the tweezer dielectrophoretically inside a cell. ${ }^{143,296}$ By retracting the nanotweezer, the molecule was extracted from the cell for subsequent analysis.

The nanotweezer consisted of a double barreled nanopipette which was modified to accommodate two carbon electrodes. It was fabricated from a double-barreled quartz capillary by laser pulling as described above. Pyrolytic deposition of carbon was used to fabricate the carbon nanoelectrodes at the tip of the nanopipette (Figure 23A). The separation between the electrodes was $\sim 10-20 \mathrm{~nm}$, as determined by the thickness of the wall dividing the two barrels of the nanopipette.

To trap the target molecule, an alternating voltage was applied at the carbon nano-electrodes $\left(20 \mathrm{~V}_{\mathrm{pp}}, 1 \mathrm{MHz}\right)$ to generate a local non-uniform electric field, which exerted an attractive force on polarizable molecules near this field and induced dielectrophoretic trapping of the molecules (Figure 23B,C). High gradients of electric fields $\left(\nabla|\mathrm{E}|^{2} \sim 10^{28} \mathrm{~V}^{2} / \mathrm{m}^{3}\right)$ were generated at the tip. ${ }^{143}$ Such high gradients were essential for extracting molecules ranging from small proteins $(<15 \mathrm{kDa})$ to DNA (below 200 base pairs) from within the cell as well as cell organelles. Due to the small separation $(\sim 10-20 \mathrm{~nm})$ between the electrodes, a small voltage (a few volts) was sufficient to generate the electric fields needed, thereby minimizing effects associated with heating. The trapping efficiency of DNA depended on the applied voltage and frequency, due to the change in polarizability of DNA molecules arising from the variation in the relaxation time constant of the ions that surround the DNA.

Selective labelling of molecules and organelles made it possible to target the molecules/organelles of interest. A micromanipulator was used to approach the cell with the nanotweezer, guided by an optical microscope. For example, target DNA was extracted from the nucleus of human bone osteosarcoma U2OS cells and human pancreatic adenocarcinoma cells (HPAC), mRNA from the cytoplasm of HPAC cells, and mitochondria from axons of primary mouse hippocampal neurons.

This technique was fast ( $<10$ s for the trapping of molecules) and minimally invasive. The functional integrity of the extracted DNA was preserved and verified by quantitative polymerase chain reaction (qPCR). Unlike nanopipettes which involve the non-specific aspiration of cytoplasmic fluid, the nanotweezer trapped molecules close to the tip only and was thus more specific. Similar to the nanopipettes, this technique allowed multiple sampling of the same cell at different time points. Cell viability was found to be unaffected by two biop- sies 16 hours after the second biopsy. The membrane damage caused by the biopsies was thus not permanent.

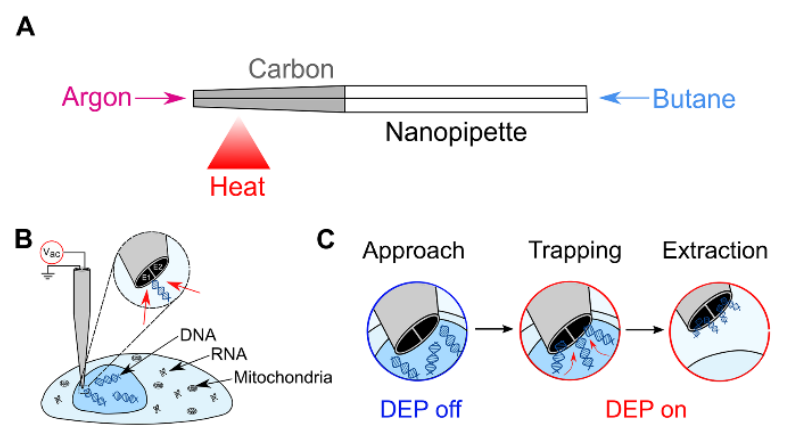

Figure 23. Biopsy using nanotweezers. (A) Integration of carbon nanoelectrodes into a double barrel nanopipette to fabricate a nanotweezer. (B) Experimental set-up showing the operation of a nanotweezer as a tool for performing single cell biopsy. (C) For biopsy, the nanotweezer first approaches the target location. This is followed by dielectrophoretic trapping of target molecules (DNA in this case), and finally withdrawal of the nanotweezer with the trapped molecules. All diagrams were adapted and redrawn from Nadappuram et al. ${ }^{143}$

\subsection{Diffusion following electroporation}

\subsubsection{Nanostraws}

Nanostraws are an array of hollow nanotubes which pierce into cells that were cultured on top. ${ }^{297}$ Application of an electrical voltage across the nanostraws causes nanoelectroporation or local permeabilization of the plasma membrane around the nanostraw. This membrane permeabilization allows the transport of cellular materials out of the cell by diffusion. ${ }^{297}$ Intracellular sampling using nanostraws has maintained high cell viability ( $>95 \%$ ) post extraction. ${ }^{297,298}$

In the first demonstration, nanostraws were made of alumina. The fabrication started with depositing a layer of alumina $(10-40 \mathrm{~nm})$ on a polycarbonate membrane with atomic layer deposition. $^{297}$ The alumina coating on the top surface of the polycarbonate membrane was then removed by directional reactive ion etching (RIE), leaving behind an alumina coating on the surface of the pores. Finally, the nanostraws were exposed by removing a desired thickness of the polycarbonate membrane by oxygen RIE. The diameter and the density of the nanostraws varied from $100-750 \mathrm{~nm}$ and $10^{6}$ to $10^{8}$ pores $/ \mathrm{cm}^{2}$, respectively, and were controlled by the diameter and the density of the pores in the polycarbonate membrane. ${ }^{297,299,300}$ The diameter of the pores could also be altered by using oxygen plasma etching. ${ }^{298}$ The height of the nanostraws were $~ 1-1.5$ $\mu \mathrm{m},{ }^{297-299}$ and the thickness of the walls of the nanostraw varied from $10 \mathrm{~nm}$ to $40 \mathrm{~nm} .^{297-299}$ The composition of the nanostraws could be varied. ${ }^{301}$ Using a similar technique, nanostraws were fabricated with metals ( $\mathrm{Pt}$ and $\mathrm{Au}$ ), a conductive polymer poly- (3,4-ethylenedioxythiophene) (PEDOT), and a metal/polymer hybrid Pt/PEDOT. The nanostraws used in this study had a thickness between $100 \mathrm{~nm}$ and $600 \mathrm{~nm}$, with a height up to $4 \mu \mathrm{m}$. To facilitate the extraction and collection of intracellular materials, the array of nanostraws was integrated with a chamber or microfluidic channel underneath containing an extraction buffer. 
Intracellular sampling using nanostraws worked on the principles of electroporation and diffusion. Cells were first cultured on the nanostraw membrane. The membrane was placed on a droplet of extraction buffer which rested on top of an indium tin oxide (ITO) electrode. The counter electrode was immersed into the cell culture buffer. For electroporation, 10$35 \mathrm{~V}$ square waves (pulse duration $200 \mu \mathrm{s}$; frequency $20 \mathrm{~Hz}$ ) were applied for 20-60 s. ${ }^{297}$ The short duration of voltage application ensured that cells were not severely damaged due to permeabilization. Diffusion of cellular material from the cell continued until the pores sealed spontaneously, which occurred between 2 and 5 mins after voltage application. ${ }^{297}$ However, the overall timescale for extraction was determined by the diffusion of molecules being extracted. Over a period of 10 minutes, it was found that $\sim 7 \%$ of the cellular red fluorescent proteins (RFP) were extracted, ${ }^{297}$ and the level of RFP extracted correlated with the level expressed in the cells at different time points. To extend the time over which molecules could diffuse from the cell, He et al. applied a lower voltage of $10 \mathrm{~V}$ at $2 \mathrm{~Hz}$ for 30 minutes. ${ }^{298}$ It was found that the cells were able to survive this prolonged poration and extraction.

Unlike extraction methods based on electrokinetic flows, nanostraws did not depend on the charge of the molecules. The diffusion process was non-specific, and a wide range of proteins, mRNA and other biomolecules have been extracted. ${ }^{297}$ However, only molecules smaller than the diameter of the nanostraws could diffuse out of the cell, and large molecules would require long diffusion times. The amount of cell content extracted increased with the diameter and/or density of nanostraws on the polymer membrane. With the array format, the nanostraws sampled cellular content from different parts of the cell simultaneously, although it would be difficult to sample specific regions of the cell.

Nanostraw extraction has been performed on different cell types including Chinese hamster ovary (CHO) cells, ${ }^{297,298}$ HeLa cells, ${ }^{301}$ and astrocytes and cardiac myocytes (CM) derived from human induced pluripotent stem cells (hiPSC). ${ }^{297}$ Different methods have been used for analyzing the extraction buffer including fluorescence (for fluorescent proteins), enzymatic assays, or polymerase chain reaction (PCR) ${ }^{297}$ Cao et al. performed a standard enzyme linked immunosorbent assay (ELISA) on the extraction buffer to detect and analyze heat shock protein 27 (HSP27) in hiPSC-MCs. ${ }^{297}$ They also performed RT-PCR to estimate the expression levels of the mRNA in the sample extracted from hiPSC-CMs. The study found that the efficiency of extraction was governed by the size of the mRNA and also their localization within the cell. Larger molecules were more difficult to extract within the time frame the plasma membrane pores were open. He et al. used ELISA to study the lactate dehydrogenase B (LDHB) protein in CHO cells. ${ }^{298}$ The study used around 18000 cells to extract the amount of LDHB required for ELISA. As the extraction process was minimally invasive and had no significant effect on cell viability, they were able to extract and analyze the cell sample at different time points over 2 hours. Wen et al. detected and analyzed the expression of caspase 3 enzyme in HeLa cells at different time points. ${ }^{301}$ Caspase 3 has a critical role in controlling cell apoptosis and hence is useful in understanding the mechanisms of cancer progression.

\subsubsection{Nanoporous membrane}

Instead of having nanotubes piercing into the cells, devices called the live cell analysis device (LCAD) used nanochannels (radius $\sim 250 \mathrm{~nm}$ ) formed by a nanoporous polycarbonate membrane, on which cells were cultured. ${ }^{302,303}$ Similar to nanostraws, LCAD operated on the principle of membrane permeabilization using electroporation followed by an outflow of cytosol primarily by diffusion. The LCAD was sandwiched between two indium tin oxide electrodes. tdTomato protein was extracted from an engineered cell line. The integration with self-assembled monolayers for matrix-assisted laser desorption/ionization mass spectrometry under the polycarbonate membrane allowed the quantification of enzymes (e.g., tyrosine phosphatase) extracted from the cells. Cell viability one day post electroporation was found to be $\sim 93 \%$ which was found to be comparable to the viability of cells which were not electroporated $(\sim 95 \%)$.

\subsection{Pressure-driven flow}

\subsubsection{Fluidic force microscopy}

Fluidic force microscopy (FluidFM) is a derivative of a conventional AFM where the cantilever contains a hollow microchannel and the tip has an aperture for fluid transfer to and from the cell. ${ }^{276,304-306}$ The microchannel is connected to a pressure controller via a macrochannel drilled to the AFM probe holder. Once the tip is inserted into the cell, the application of a negative pressure to the microchannel withdraws fluids from the cell.

FluidFM cantilevers were fabricated similarly to an AFM cantilever. Silicon wafers were etched to create cavities which were then lined with silicon dioxide. ${ }^{304}$ Selective silicon etch produced free-standing silicon dioxide structures, forming a hollow glass cantilever attached to a silicon chip. An aperture at the cantilever tip was opened using focused ion beam milling (FIB). The microfluidic channel inside the cantilever had a cross section of $2 \mu \mathrm{m} \times 10 \mu \mathrm{m} .{ }^{304}$ Prior to extraction, the microchannel was coated with an anti fouling agent (poly(Llysine)-graft-poly(ethylene glycol)) and filled with an aqueous buffer (phosphate buffered saline).

The FluidFM benefited from the precision of a conventional AFM. Specific sites on the cell were targeted and approached with a high degree of precision by virtue of the sensitive AFM force feedback. For example, Guillaume-Gentil et al. were able to target the cytoplasm and the nucleus of HeLa cells using FluidFM and selectively extract contents from the cytoplasm and the nucleus. ${ }^{305}$ Using a $400 \mathrm{~nm}$ aperture tip and a negative pressure of $-0.8 \mathrm{~atm}, \mathrm{mRNA}$, soluble proteins, vesicles, and cytoskeletal fibers smaller than the aperture were extracted. Larger proteins and subcellular organelles such as mitochondria could not pass through the aperture and were excluded. The volume of cell content extracted ranged from $0.1 \mathrm{pL}$ to $7.0 \mathrm{pL}$. The maximum flow rate achieved in their study was $0.4+/-0.1 \mathrm{pL} / \mathrm{min}$. Therefore, FluidFM allows rapid extraction of large volumes of cellular content.

The viability of the cells post sampling depended on the volume of the sample extracted and the site of extraction. The study by Guillaume-Gentil et al. found that cells remained largely viable when cytoplasm samples up to $4.0 \mathrm{pL}$ and nucleoplasm samples up to $0.6 \mathrm{pL}$ were collected, corresponding to $90 \%$ and $20 \%$ of the native cytoplasmic and nuclear volumes, respectively. 


\subsubsection{Extended nanochannel}

Lin et al. reported an extended nanochannel coated with lipid bilayer which worked as a femtoliter pipette for sampling from a live cell after the cell membrane fused with the lipid bilayer at the junction of the nanochannel. ${ }^{307}$

The device consisted of two parallel microfluidic channels for trapping single cells and for collecting cellular extracts, respectively. The two microfluidic channels were connected by an extended nanochannel $(900 \mathrm{~nm}$ wide $\mathrm{x} 900 \mathrm{~nm}$ tall x 50 $\mu \mathrm{m}$ long). The microchannels were fabricated on a glass substrate using photolithography and dry etching. The extended nanochannel was fabricated on a separate fused silica glass substrate by electron-beam lithography and plasma etching. After bonding the channels to a glass bottom, a lipid bilayer was formed on the wall of the extended nanochannel by introducing vesicles followed by vesicle fusion.

During operation, a cell was first trapped in the single cell chamber. A pressure above $60 \mathrm{kPa}$ was applied to cause the cell membrane to fuse with the lipid bilayer coating the surface of the nanochannel. The exact mechanism of fluid flow out of the cell was not described explicitly, but it was likely driven by pressure gradient across the nanochannel. A volume of $39 \mathrm{fL}$ of cell cytoplasm was sampled. After cytoplasm extraction, the cells were released by application of a high pressure of over $400 \mathrm{kPa}$ from the opposite direction. This device was used to sample the cytoplasm of a living human aortic endothelial cell (HAEC). Cells were found to be viable 12 hours post extraction.

\subsection{Outlook and future opportunities}

The micro and nanofluidic single-cell biopsy techniques described above have opened doors to sampling contents from live cells in real time. These techniques are versatile and have been shown to work well in a wide range of cell types. Depending on the specific device geometry, the sampling of a single cell or many cells can be achieved. The small size of the tip or aperture are minimally invasive to the cells. The spatial distribution of the organelles and the cytosol are not affected during the sampling process, making it possible to sample and identify local inhomogeneities within the cell. Since these techniques do not affect cell viability significantly, the same cell can be sampled repeatedly over long periods of time. Although the samples collected are typically non-specific, many techniques are available to separate and analyze the extracted biomolecules in vitro.

A challenge of most single-cell biopsy methods is the low throughput, since only one cell can be sampled at a time. Diffusion mediated techniques such as the nanostraws can sample thousands of cells at a time. However, it takes a long time ( 10-30 minutes) to obtain samples of reasonable yield. Nevertheless, it is possible to increase the throughput of biopsy for device geometries that are amenable to integration with parallel micro or nanochannels on a chip, such as the extended nanochannels described by Lin et al. ${ }^{307}$ Robotic systems can also be used to increase the throughput. ${ }^{270,308}$ Another challenge of some high precision biopsy techniques is that they can only work with adherent cells. However, along with on-chip suction valves or immobilization methods described in Section 4.3, it should be possible to extend these methods to an even broader range of cell types.
Finally, current biopsy tools can be further enhanced by direct integration with sample analysis. For example, Liu et al. developed a microfluidic "T probe" capable of rapid cytosol extraction (on the order of seconds) which is fed directly to a mass spectrometer. ${ }^{309}$ In this work, the $\mathrm{T}$ probe extracted a large volume of cytosol which resulted in low cell viability. The experimental parameters and runtime could be optimized, however, to increase cell viability. 


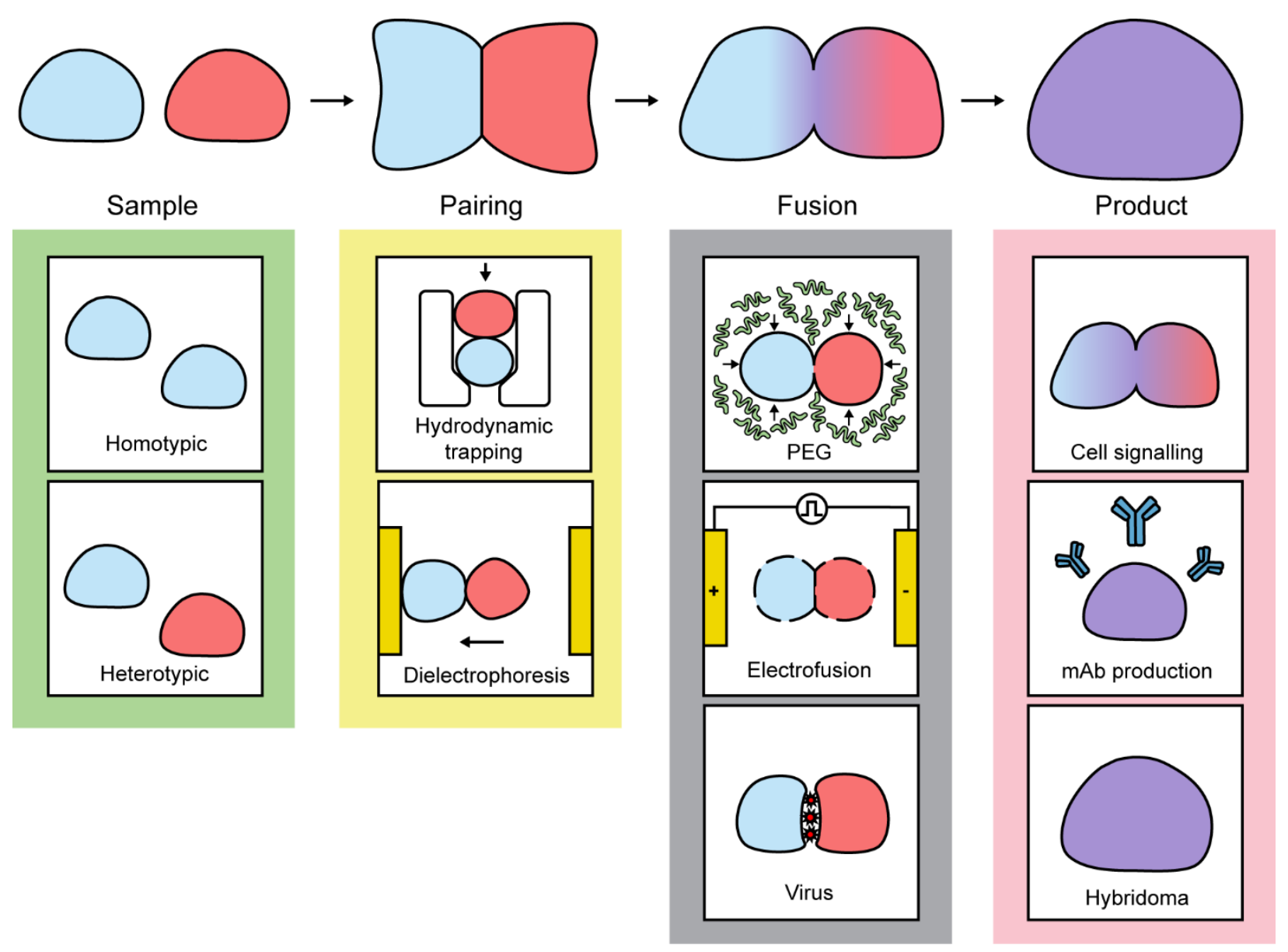

Figure 24. Fusion surgery workflow. Fusion surgery consists of choosing a sample, a method for pairing, and a method for fusion. The resulting product can be used for a variety of downstream applications.

\section{FUSION}

We define fusion as the process of joining two or more units to form a single entity. This process is applied primarily to the fusion between two single cells of the same cell type (homotypic fusion) or different cell types (heterotypic fusion). In vivo fusion between cells is a naturally occurring phenomenon with important biological functions. ${ }^{310}$ For example, the fusion of muscle precursor cells is necessary for the proper development of skeletal muscle, ${ }^{311,312}$ the fusion of sperm with egg is vital for fertility, ${ }^{313}$ and the fusion of epithelia is important for normal development. ${ }^{314}$ Disruption of normal cell fusion processes can lead to infertility, cancer, preeclampsia, muscle diseases, and parasitic, bacterial, and viral infections. ${ }^{315}$

For in vitro fusion studies, the most important practical application is perhaps hybridoma technology, where B cells are fused with myeloma cells to form hybridomas, which can produce monoclonal antibodies for a broad range of diagnostic and therapeutic applications. ${ }^{316}$ Other applications of fusion include the study of cell-cell interaction and the mechanisms regulating gene expression, ${ }^{317}$ investigation of cellular reprogramming, ${ }^{318-320}$ and the development of a cancer vaccine. ${ }^{321}$

In the past, in vitro fusion studies required manual surgery. For example, in the fusion or grafting between two Stentor coeruleus, the two cells were cut open by a sharp needle and the wound surfaces were pressed together firmly to fuse them in a viscous liquid. ${ }^{163}$ One of the first methods of cell fusion that did not require direct manual surgery was the use of Sendai virus in the 1960s. ${ }^{322}$ It was followed by the discovery of polyethylene glycol (PEG)-mediated fusion in the $1970 \mathrm{~s}^{323}$ and electrofusion in the 1980s. ${ }^{324}$ However, all of these methods suffer from low cell pairing accuracy and low fusion efficiencies. To address these challenges, microfluidics methods have been developed to trap a large number of cell pairs with high precision. In combination with existing fusion techniques, microfluidic fusion surgery has achieved drastic improvements in fusion efficiencies. The general workflow of these experiments can be seen in Figure 24. In this section, we will describe microfluidic fusion methods, most of which are on single cells but with recent examples on multicellular systems.

\subsection{Single cells}

At the single cell level, fusion involves the merging of two (or more) cells. A key advantage of microfluidics is the superb ability to trap cells and keep them in contact and immobilized for subsequent fusion. Many microfluidic methods for trapping have been reported, including hydrodynamic trapping, dielectrophoretic trapping, magnetic trapping, and acoustic trapping. ${ }^{325}$ Below, we will focus on trapping methods that have been used in conjunction with fusion. 


\subsubsection{Polyethylene glycol (PEG)-mediated fusion}

PEG has been used widely as an agent for the aggregation and fusion of cells. However, controlling single cell pairings in bulk suspension is difficult, resulting in poor fusion efficiency. Elegant microfluidic methods have been developed to address this challenge by trapping and pairing thousands of cells before fusion. After fusion, PEG can also be washed off quickly to minimize cytotoxicity.

The microfluidic device described by Skelley et al. is one of the early seminal works that demonstrated the advantage of microfluidics in achieving a high efficiency in cell pairing and fusion. ${ }^{326}$ Multiple cell types were tested, including NIH3T3 fibroblasts (3T3), myeloma cells, B cells, mouse embryonic stem cells (mESCs), and mouse embryonic fibroblasts (mEFs). The device consisted of a dense array of $\sim 6000$ weir-based passive hydrodynamic cell traps. Each trap had a large capture cup and a small capture cup facing in opposite directions. The loading protocol had three steps. In step one, the first cell type was introduced by flow in the upward direction. They were trapped by the small capture cups on the back of each trap. In step two, a downward flow was applied. The trapped cells were transferred into the large capture cups of the opposing traps, which had space for two cells only. In step three, the second cell type was introduced by downward flow and became trapped by the large capture cups, thereby saturating the trap and preventing more than two cells to be loaded in each trap. The pairing efficiency was up to $70 \%$. Flowing PEG solution past the cells did not dislodge them from the traps. PEG fusion took 25 minutes for cells to fully merge with $\sim 39 \%$ fusion efficiency. This study also tested electrofusion, which took 20 minutes for cells to merge with $\sim 78 \%$ fusion efficiency. The hybrid cells were retrieved. Reprogrammed mEFs after fusion with mESCs were observed after 14 days of culture. Over the entire device, $>50 \%$ properly paired and fused cells was achieved, representing a fivefold improvement over commercial electrofusion chambers.

In a follow-up work by the same group, deformable traps and changes in osmolarity were used to enhance the immobilization of cell pairs within the traps. ${ }^{327}$ This design enabled rapid media exchange, and was compatible with chemical, biological, and physical fusion protocols. Approximately 750900 traps were loaded in $<60$ seconds. The pairing efficiency was up to $80 \%$. Fusion with PEG, virus, and electrofusion were tested. The fusion efficiency was up to $95 \%$ (for electrofusion).

Other studies on PEG-mediated fusion used similar ideas to trap cells first and then fuse them. For example, Wu et al. reported a device consisting of double micro-column lines to trap and fuse tobacco mesophyll protoplasts using PEG. ${ }^{328}$ This study was one of the first to demonstrate fusion of plant cells in a microfluidic system. Since this device relied on cells that were in close contact when PEG was introduced, the fusion efficiency was $28.8 \%$ only. In a separate study by Grabowski et al., a microfluidic trap and a pneumatic valve were used to actuate the flow to bring cells into contact, followed by the introduction of PEG to induce fusion. ${ }^{329}$ The cells tested were human myeloid cell line U937 and human lymphoid cell line L540. No pairing or fusion metrics were reported, however.

\subsubsection{Electrofusion}

As can be seen from the study by Skelley et al., ${ }^{326}$ electrofusion efficiency is generally higher than PEG-mediated fusion. Another advantage of electrofusion is that the electric field can also be used to trap cells based on dielectrophoresis. The first microfluidic device to electrofuse single cells was described by Wang and Lu. ${ }^{330}$ The device consisted of a microfluidic channel with narrow and wide sections. The electric field could then be controlled such that the field in the narrow sections of the device was high enough for cell fusion to occur, and the field in wide sections were too weak to have adverse effects on cell viability. The success of this work generated interest for electrofusion in a microfluidic platform. The overall design concepts of subsequent microfluidic electrofusion devices are similar, differing primarily in the design of the traps and the electrodes. Below, we will highlight a few examples.

\subsubsection{Microorifice}

The microorifice design consists of a small opening, across which a cell pair is trapped and becomes fused. Gel et al. described a device with microorifices (diameter $2-10 \mu \mathrm{m}$ ) on a vertical wall inside a microchannel. ${ }^{331}$ The electrodes were patterned parallel to the wall. Cells were loaded into the device on the opposing sides of the microorifice. Dielectrophoretic force attracted the cells to the orifice to form a pair. Application of an electric field $(0.1-0.2 \mathrm{~V} / \mathrm{cm})$ initiated cell fusion. Cytoplasmic transfer was visualized by Calcein AM fluorescence of cells.

The same research group went on to quantify the efficiency of this method. ${ }^{332}$ They modified their design to eliminate the need for a pump to facilitate cell loading. In this particular device, cells were introduced into wells on both sides of microorifices. The device was tilted so that a hydrostatic pressure difference between both inlets created a flow that carried the cells to one side of the microorifices. An applied AC field enabled trapping of the cells at the microorifices by dielectrophoresis. Only cells at the microorifice were held in place and other cells were able to be washed away. Once all the cells were paired, an electric field was applied to cause fusion. The fusion sequence composed of an AC field (10 Vpeak-to-peak, $1 \mathrm{MHz})$ for $10 \mathrm{~s}$, and then a DC pulse $(4 \mathrm{~V}, 300 \mu \mathrm{s})$ superimposed on the AC field for another $10 \mathrm{~s}$. A pairing and fusion efficiency of $>95 \%$ was achieved. In a follow up work by the same group, their microorifice design was adapted into a large planar array format, ${ }^{333}$ increasing the number of cells that can be fused to $10^{4}-10^{6}$ cells in a single device. An improved microorifice design also allowed the pairing of cells with different sizes easily. A fusion efficiency of $78-90 \%$ was reported.

One of the most interesting aspects of the microorifice design is that the size of the microorifice controlled whether nuclear mixing occurs or not. Nuclear mixing could lead to abnormal karyotypes. Cell fusion and cytoplasmic transfer without nuclear mixing could thus allow the induction of transdifferentiation and reprogramming keeping the karyotype normal. $^{334}$

\subsubsection{Microelectrode arrays}

Microelectrode arrays have been incorporated with microfluidics for high-throughput cell pairing by dielectrophoresis and electrofusion. ${ }^{335-338}$ The key advantage of this system 
compared with microorifice design was that the fused cells can be retrieved immediately by turning off the electric field and flowing the cells out of the device.

In one study, a microfluidic electrofusion device was developed consisting of a microelectrode array made of doped silicon. ${ }^{336}$ The electrode array also worked as the sidewalls of the microchannels $(42 \mu \mathrm{m}$ tall $\times 100 \mu \mathrm{m}$ wide). Each electrode had square protrusions $(20 \mu \mathrm{m} \times 20 \mu \mathrm{m})$ spaced $60 \mu \mathrm{m}$ apart to create high electric field gradients for cell trapping. The region with a high gradient field was $<8 \mu \mathrm{m}$. As such, the trapping of more than two cells (with a radius of 4-8 $\mu \mathrm{m}$ ) was unlikely. Human Embryonic Kidney 293 cells (HEK293), mouse fibroblasts (NIH3T3), and mouse embryonic stem cells were tested. Pairing efficiency of different cell types was $\sim 35 \%$. The electric field needed for fusion was $\sim 1000 \mathrm{~V} / \mathrm{cm}$ and the fusion efficiency was $\sim 46 \%$. Cell alignments took $\sim 1$ minute, and the electrofusion of paired cells was achieved within 3 minutes of pulsing. The cell viability was $>90 \%$ for voltages up to $9 \mathrm{~V}$.

One of the disadvantages of this work was that cells were trapped in the space between adjacent protruding microelectrodes, referred to as the dead zones. A follow up work from the same group addressed this issue by filling the gaps with a dielectric. ${ }^{338}$ More than $99 \%$ of cells were trapped and aligned at the edges of the microelectrodes and $\sim 70 \%$ of cell chains contained only 2 cells. However, this design still could not address another drawback of the protruding microelectrode design, which was the difficulty to control the pairing of different cells for hybrid production.

\subsubsection{Droplet encapsulation}

Previous methods of cell pairing based on dielectrophoresis often suffer from the trapping and fusion of more than two cells. The generation efficiency of functional hybridoma is also unclear. ${ }^{339-344}$ To attempt to address these challenges in part, Schoeman et al. described a microfluidic device that trapped cells and electrofused them inside microdroplets. ${ }^{345} \mathrm{~A}$ flow-focusing nozzle was used to generate drops from a cell suspension having a volume fraction of $1 \%$. By Poisson statistics, $15 \%$ of the drops contained two cells. The drops passed over six recessed platinum electrode pairs for electrofusion. Six voltage pulses of 2-3 V were applied. The system had a high throughput and could process 500 cells/second. However, the fusion efficiency was only $5 \%$. The advantage of this system, however, was that it is directly compatible with other high throughput droplet microfluidics operations downstream.

\subsubsection{Cell traps}

The most reliable method to ensure the pairing and trapping of two cells before fusion was demonstrated by using cell traps that can fit two cells at a time. The seminal studies by Skelley et al. ${ }^{326}$ and Dura et al. ${ }^{327}$ described in Section 6.1.1 also demonstrated electrofusion, where the electrodes were fabricated outside of the chamber containing the array of hydrodynamic traps.

In another study, Yoshimura et al. reported a device with an array of 10,000 microwells that can trap and pair cells vertically with positive dielectrophoretic force. ${ }^{346}$ The microwells were sandwiched between upper and lower indium tin oxide electrodes. These microwells were designed to hold two vertically aligned mouse myeloma cells. Different cell populations were introduced separately and trapped in microwells by a dielectrophoretic force. The time required for the formation of the array of cell pairs was less than a minute (over 5,00 pairs made). The pairing efficiency was $\sim 50 \%$, but no fusion metrics were reported. Fused cells could easily be collected after removing the electric field.

He et al. described a device that integrated cell pairing, fusion, and long-term culture of HeLa cells on the same chip. ${ }^{347}$ The device consisted of two parts. The lower part (device floor), consisting of an array of trenches with electrodes patterned underneath, was responsible for cell capture, pairing, and fusion. Positive dielectrophoretic force was applied to trap cells into the trenches. Excess cells were washed off. Negative dielectrophoretic force was then applied to bring the trapped cells into contact. An electric field of $20 \mathrm{kV} / \mathrm{cm}$ (40 ms duration, 5 pulses, $0.5 \mathrm{~s}$ interval) was applied to fuse the trapped cells. The upper part of the device (device ceiling) was designed to be a cell culture well. It was made of PDMS modified with fetal bovine serum for extended cell culture. After cells fused, the device was flipped upside down, transferring the fused cells to the device ceiling culture. Switching the dielectrophoresis buffer with culture media allowed for cell growth of the newly fused cell pair. Filling the trap array, pairing, and fusion required only 5 minutes. This device generated a total of 864 homotypic cell pairs, with a pairing efficiency of $\sim 78 \%$ and a fusion efficiency of $\sim 26 \%$. Successful cell proliferation and migration were demonstrated 72 hours after onchip culture. However, long-term culture of hybrid cells on chip remains difficult to achieve with similar efficiencies as homotypic fusions.

\subsubsection{Virus-mediated fusion}

Although less common than electrofusion and PEG-mediate fusion, virus-mediated fusion has well established biology and other unique advantages (see details in Section 2.2.1). ${ }^{348}$ Since the virus is introduced in solution, in principle, the devices developed for PEG-mediated fusion could also be applied for virus-mediated fusion.

Sasaki et al. described a microfluidic viral fusion device with a single pairing step. ${ }^{349}$ Different cell suspensions were added simultaneously from separate inlets to the microchannels consisting of a microslit with a hydrodynamic weir. The pairing and fusion of cells were studied near the microslit. Using embryonic stem cells (B6G-2) and somatic cells (3T3), the device was able to achieve a pairing efficiency of $80 \%$. HVJ envelope was introduced for fusion. The fusion efficiency depended on the slit size: it was $\sim 17 \%$ for a $10 \mu \mathrm{m}$ microslit and $\sim 6 \%$ for a $2 \mu \mathrm{m}$ microslit, respectively.

Also using the concept of microslits, Wada et al. described a device to pair and fuse single cells at the microslit. ${ }^{334}$ The goal was to allow cytoplasmic transfer between the trapped cells but prevent nuclear mixing, so that the induction of transdifferentiation and reprogramming could be studied while keeping the karyotype normal. Here, cell fusion was induced by the HVJ envelope. This method created a stricture in the cytoplasmic connection. The microslit prevented nuclear mixing in spread shaped NIH3T3 cells, but not round shaped NIH3T3 cells. Mitochondria was shown to be transferred to the recipient cell. 


\subsection{Multicellular systems}

At the multicellular level, microfluidic fusion surgery demonstrated so far has involved the joining of two or more spheroids. Applications include the fusion of tumor spheroids with fibroblast spheroids to study metastatic characteristics. The study of fusion in multicellular systems in vitro is less explored than in single cell systems. The role of microfluidics is to bring different spheroids in close contact with each other in a controlled, high throughput manner. These studies are motivated by the need to recapitulate the $3 \mathrm{D}$ microenvironment to model physiological and pathological behavior in vivo.

Tocchio et al. described the use of magnetic levitation in a capillary to control the merging of different spheroids. ${ }^{350}$ The motivation was to biomanufacture functional living systems to model physiological and pathological behavior. A gadoliniumbased nonionic paramagnetic agent was used with magnetic fields to levitate and assemble cells and spheroids. The magnetic force directed the spheroids toward the minimum of the magnetic field, allowing them to interact and eventually fuse. The effect of surface tension and spheroid composition were studied on the 3D arrangement of the fused product. The spheroids were viable for over 4 days. The throughput of the system was not reported, but appeared to be relatively low.

Tomasi et al. developed a microfluidic platform with a large array of asymmetric anchors to pair and merge drops containing different spheroids. ${ }^{351}$ The motivation of the work was to control the microenvironment of $3 \mathrm{D}$ cultures of spheroids for studying tissue self-organization and drug testing. The array consisted of asymmetric anchors. The large anchor was used to trap the first set of drops with one type of cells which were allowed to settle and form a spheroid. A second set of drops containing another cell type was then introduced and trapped in the small anchor adjacent to the first drop. They were again allowed to settle and form a spheroid. Finally, flushing a destabilization agent in the outer phases caused the coalescence of the two droplets and the fusion of the two types of spheroids.

Zhao et al. developed a simple method based on microwell arrays and centrifugation for fusing tumor and fibroblast spheroids. ${ }^{352}$ The motivation was to reconstruct the heterogeneous tumor microenvironment comprising both stromal and tumor components and to study 3D metastasis. Tumor and stromal cells were seeded separately into two microwell arrays to generate tumor and stromal spheroid cultures. Tumor spheroids were collected with a pipette and transferred into the array with stromal spheroids, which had a depth of approximately the size of two spheroids. The device was centrifuged down for pairing of tumor and stromal spheroids. This process achieved a pairing efficiency of $\sim 78 \%$ for 240 tumor-stroma pairings. Two different tumor spheroids, generated from colon cancer cell line HCT-116 and breast cancer cell line MCF-7 respectively, were compared for metastatic characteristics by observing the interactions between the tumor spheroid with a fibroblast spheroid. HCT-116 spheroid fused with fibroblast spheroids within 5 hours after pairing, while it took 7 hours for MCF-7 to fuse with fibroblast spheroid, suggesting that HCT-116 spheroids were more invasive than MCF-7.

\subsection{Outlook and future opportunities}

In summary, microfluidic methods have drastically improved the efficiency of fusion in both single cell and spheroid systems compared with methods in bulk. Fusion surgery has leveraged the unique advantages of microfluidics to control the trapping and pairing of cells or spheroids with high precision. Most studies to date have focused on mammalian cells. It would be of interest to see if microfluidic methods can be extended to other systems, such as Stentor ${ }^{353,354}$ and colonial tunicate Botryllus schlosseri, ${ }^{355}$ which are classic models on grafting and regeneration. In addition, current applications of microfluidic fusion surgery did not require the control of the orientation of entities to be fused, since the cells or spheroids were relatively uniform. For the fusion or grafting of organisms like Stentor or Botryllus, the specific location of the graft is important and can determine the subsequent regeneration potential and pathway. In this case, imaging and the control of animal orientation will be required prior to fusion. Although this capability has not been demonstrated, the microfluidic techniques developed for animal immobilization for laser ablation surgeries (Section 4.3) could well be applied here.

\section{SUMMARY AND PERSPECTIVES}

\subsection{Summary}

In summary, the field of microscale surgery has evolved from performing the surgery by hand, to the use of microfluidics integrated with optical, electrical, and chemical techniques to perform the surgery in a fully or semi-automated manner. Microfluidic surgery has drastically improved the precision and the throughput of surgical operations, the range of cell types, tissues and organisms that can be examined and manipulated, as well as the post-surgery viability of the samples.

The precision of microfluidic surgery in cutting a specific cellular structure is provided by a combination of many factors, including the plethora of methods to immobilize and to position the sample in microfluidic systems, the ability to fabricate sharp micro or nanoknives and integrate them on-chip, and the use of femtosecond lasers along with advanced microscopy. The cut can thus be localized to small regions of interest.

The high throughput of microfluidic surgery is made possible in two ways. The first is the ability to flow a continuous stream of samples through the surgical areas for operations performed in a serial manner. Laser ablation of specific cell types inside live organisms, and the bisection of cells or organoids in the microfluidic guillotine are two examples where the throughput is increased this way. The second way that the throughput is increased is by using a massively parallel array of traps to immobilize many samples at once so that the surgical operation can be performed to all samples at the same time. The separation of daughter cells from mother cells, and the fusion of many cell pairs are two prominent examples.

The types of samples that can be operated on are no longer limited to those that are large enough to be dissected by hand. Nevertheless, most microfluidic surgery have been applied to mammalian cells and popular model organisms such as $C$. elegans, largely due to their biomedical applications.

The improved post-surgery viability in microfluidic systems is a direct result of the precision of the surgery, since the damage is limited to a very small area. In addition, the fine control of the microenvironment of the surgery, as well as the use of physical immobilization instead of anesthetics during surgery have both facilitated fast recovery of the organisms after the surgery. 


\subsection{Perspectives}

Since we have already provided concluding remarks specific to each type of surgery at the end of each section in Sections 3-6, we will not repeat the comments here. Rather, we will point out a few challenges and opportunities common to multiple types of surgery below.

Fundamentally, Section 2 has revealed gaps in some of the principles of surgery, in particular, the interaction between mechanical and optical forces and biological materials. A better understanding of the underlying mechanisms can facilitate better designs of surgical tools to increase the precision of surgery and further minimize undesired damage to the cells or tissues associated with the surgery. It can also enable the characterization or prediction of the biomechanical properties of the cells or tissues and their corresponding physiological state, by measuring their response to the surgical tool.

In terms of the potential uses of microfluidic surgery, we are only scratching the surface. For example, organisms such as the unicellular ciliate Stentor coeruleus, the planarian flatworm, and the colonial tunicate Botryllus are classic models of regeneration and have traditionally been studied by dissection or grafting by hand. ${ }^{356,357}$ With the exception of the microfluidic guillotine to bisect Stentor, few microfluidic surgical techniques have been applied to study these organisms. We believe that the methods described in this review can be adapted to further elucidate the mechanisms underlying regeneration in these organisms. However, innovations in microfluidic designs to immobilize, position, cut, or graft the organisms may be needed since these organisms are either highly motile, or have different shapes and stiffness from the cells and organisms that current microfluidic surgery have been developed for.

Another potential use of microfluidic surgery is in combination with cell atlas and computer vision to further automate the surgery. One of the challenges in dissection or ablation surgery is the identification of the target structure, which relies critically on optical imaging. Leveraging recent developments in cell atlas providing rich cell position and lineage information, ${ }^{358}$ along with machine learning and real-time feedback control, it should be possible to further increase the level of automation and throughput of microfluidic surgery. Such capability has potential to expand the phenotypes that can be studied with high statistical power to elucidate the functional roles of different cell types within the organism. The same notion applies to subcellular structures. Nanobiopsy can enable the mapping of organelles and other subcellular structures with high spatiotemporal resolution. Such information can in turn facilitate the dissection or ablation of these structures to identify their functional roles with the cell.

Finally, the surgical operations we have covered in this review are limited to samples that are smaller than, and are completely enclosed by, the microfluidic system. Recently, microfluidics have also been applied to microneedles, ${ }^{359} \mathrm{mi}$ crogrippers, ${ }^{360}$ and soft robots. ${ }^{361}$ It is therefore foreseeable that microfluidics can be extended to performing surgery on macroscale organisms like large animals or even humans, where the microfluidic system is smaller than, and can possibly be engulfed by, the organism.

\section{AUTHOR INFORMATION}

\section{Corresponding Author}

*E-mail: sindy@stanford.edu

\section{Author Contributions}

The manuscript was written through contributions of all authors. All authors have given approval to the final version of the manuscript. ${ }^{1}$ These authors contributed equally.

\section{Funding Sources}

This work was supported by funding from the Stanford Bio-X Interdisciplinary Initiatives Seed Grants Program (IIP) (No. R1014); National Science Foundation (NSF Award No.1938109); and the Center for Cellular Construction, which is a Science and Technology Center funded by the National Science Foundation (NSF Award No. DBI-1548297).

\section{Biographies}

Kevin S. Zhang is a Ph.D. candidate at Stanford University in the lab of Dr. Sindy Tang (Dept. of Mechanical Engineering). His research focuses on investigating single-cell wound healing phenomena in Stentor coeruleus using microfluidic approaches. He received his M.S. from Stanford University and his B.S. from the University of California, Los Angeles.

Ambika V. Nadkarni is a joint postdoctoral scholar in the labs of Dr. Sindy Tang (Dept. of Mechanical Engineering, Stanford University) and Dr. Wallace Marshall (Dept. of Biochemistry \& Biophysics, UCSF), where she studies the molecular mechanisms of wound-healing in the ciliate, Stentor coeruleus. She received her Ph.D. from the University of Illinois at Urbana-Champaign (UIUC) at the Department of Cell \& Developmental Biology under the guidance of Dr. William M. Brieher. Her expertise is in cytoskeletal systems. In her Ph.D. she investigated the mechanisms of actin filament disassembly by cellular factors, after which she did postdoctoral work with Dr. Rebecca Heald at the University of California, Berkeley. In Dr. Heald's lab, she studied microtubule organization in muscle cells, supported by the American Heart Association.

Rajorshi Paul is a Ph.D. student in the Mechanical Engineering Department at Stanford University. His research in the lab of Dr. Sindy KY Tang focuses on developing microfluidic tools for cutting cells to study wound healing and regeneration in single cells. He received his MSc in Mechanical Engineering from the University of Alberta, Canada where he investigated the phenomenon of inter-colony interactions in solid bacterial cultures. He received his BTech in Mechanical Engineering from the Indian Institute of Technology, Kharagpur, India.

Adrian M. Martin is a research fellow in the lab of Dr. Sindy Tang (Dept. of Mechanical Engineering, Stanford University), where he studies calcium signaling during wounding in the ciliate, Stentor coeruleus. He received his M.S. from San Francisco State University at the Department of Cell and Molecular biology under the guidance of Dr. Wilfred Denetclaw. His expertise is in developmental biology. In his M.S. degree, he investigated the mechanisms of nitric oxide generation during chicken embryo development.

Sindy KY Tang is an Associate Professor of Mechanical Engineering and by courtesy of Radiology (Precision Health and Integrated Diagnostics) at Stanford University. She received her Ph.D. from Harvard University in Engineering Sciences under the supervision of Prof. George Whitesides. Her lab at Stanford 
works on the fundamental understanding of fluid mechanics and mass transport in micro-nano systems, and the application of this knowledge towards problems in biology, rapid diagnostics for health and environmental sustainability. The current areas of focus include the flow physics of confined micro-droplets using experimental and machine learning methods, interfacial mass transport and self-assembly, and ultrahigh throughput optomicrofluidic systems for disease diagnostics, water and energy sustainability, single-cell wound healing and regeneration studies.

\section{ACKNOWLEDGMENTS}

We are grateful for insightful discussions with Prof. Wei Cai, Prof. Lakshminarayanan Mahadevan, Prof. Wallace Marshall, members of Sindy Tang's lab, and members of the NSF Center for Cellular Construction.

\section{REFERENCES}

(1) Cole, F. J. History of micro-dissection. Proc R Soc Lond, B, Biol Sci 1951, 138, 159-187.

(2) Cobb, M. Malpighi, Swammerdam and the colourful silkworm: replication and visual representation in early modern science. Ann. Sci. 2002, 59, 111-117.

(3) Malpighi, M. Marcelli Malpighii Opera posthuma : figuris aneis illustrata: quibus praefixa est eiusdem vita a seipso scripta.; 1967; p. 77.

(4) Hildebrand, E. M. Micrurgy and the plant cell. Bot. Rev. 1960, 26, 277-330. cell wound repair studies. Proc. Natl. Acad. Sci. USA 2017, 114, 7283-7288.

Zhang, K. S.; Blauch, L. R.; Huang, W.; Marshall, W. F.; Tang, S. K. Y. Microfluidic guillotine reveals multiple timescales and mechanical modes of wound response in Stentor coeruleus. BMC Biol. 2021, 19, 63.

Barber, M. A. A New Method of Isolating Microorganisms. J. Kans. Med. Soc. 1904, 4, 487.

Barber, M. A. The rate of multiplication of bacillus coli at different temperatures. Journal of Infectious Diseases 1908, 5, 379-400.

Barber, M. A. On heredity in certain microorganisms. Z.Ver-erbungslehre 1908, 1, 261-261.

Barber, M. A. A Technic for the Inoculation of Bacteria and Other Substances into Living Cells. J. Infect. Dis. 1911, 8, 348-360.

Barber, M. A. The Pipette Method in the Isolation of Single Microorganisms and in the Inoculation of Substances into Living Cells. Philippine Jour. Sc., Sec. B, Trop. M 1914, 9, 307.

E. Leitz Inc., New York. Pamphlet No. 1066. Dr. Chambers' Micro-Manipulator: an apparatus for Microtechnique or Micrurgy. E. Leitz Company Trade Literature (A copy of this pamphlet survives in Countway Medical Library, Harvard University, call no. 3.J.1926.1). 1926,

Whitesides, G. M. The origins and the future of microfluidics. Nature 2006, 442, 368-373.

El-Ali, J.; Sorger, P. K.; Jensen, K. F. Cells on chips. Nature 2006, 442, 403-411.

Squires, T.; Quake, S. Microfluidics: Fluid physics at the nanoliter scale. Rev. Mod. Phys. 2005, 77, 977-1026.

Beebe, D. J.; Mensing, G. A.; Walker, G. M. Physics and applications of microfluidics in biology. Annu. Rev. Biomed. Eng. 2002, 4, 261-286.
Stone, H. A.; Stroock, A. D.; Ajdari, A. ENGINEERING FLOWS IN SMALL DEVICES. Annu. Rev. Fluid Mech. 2004, 36, 381-411.

Duncombe, T. A.; Tentori, A. M.; Herr, A. E. Microfluidics: reframing biological enquiry. Nat. Rev. Mol. Cell Biol. 2015, 16, 554-567.

Weibel, D. B.; Whitesides, G. M. Applications of microfluidics in chemical biology. Curr. Opin. Chem. Biol. 2006, 10, 584-591.

Stewart, M. P.; Langer, R.; Jensen, K. F. Intracellular delivery by membrane disruption: mechanisms, strategies, and concepts. Chem. Rev. 2018, 118, 7409-7531.

Evans, E.; Heinrich, V.; Ludwig, F.; Rawicz, W. Dynamic tension spectroscopy and strength of biomembranes. Biophys. J. 2003, 85, 2342-2350.

Xie, X.; Xu, A. M.; Angle, M. R.; Tayebi, N.; Verma, P.; Melosh, N. A. Mechanical model of vertical nanowire cell penetration. Nano Lett. 2013, 13, 6002-6008.

Sen, S.; Subramanian, S.; Discher, D. E. Indentation and adhesive probing of a cell membrane with AFM: theoretical model and experiments. Biophys. J. 2005, 89, 32033213.

Evans, E. A.; Waugh, R.; Melnik, L. Elastic area compressibility modulus of red cell membrane. Biophys. J. 1976, 16, 585-595.

Verma, P.; Wong, I. Y.; Melosh, N. A. Continuum model of mechanical interactions between biological cells and artificial nanostructures. Biointerphases 2010, 5, 37-44.

Lulevich, V.; Zimmer, C. C.; Hong, H.; Jin, L.; Liu, G. Single-cell mechanics provides a sensitive and quantitative means for probing amyloid-beta peptide and neuronal cell interactions. Proc. Natl. Acad. Sci. USA 2010, 107, 13872-13877.

Lulevich, V.; Zink, T.; Chen, H.-Y.; Liu, F.-T.; Liu, G.-Y. Cell mechanics using atomic force microscopy-based single-cell compression. Langmuir 2006, 22, 8151-8155.

Eid, J.; Razmazma, H.; Jraij, A.; Ebrahimi, A.; Monticelli, L. On Calculating the Bending Modulus of Lipid Bilayer Membranes from Buckling Simulations. J. Phys. Chem. B 2020, 124, 6299-6311.

(30) Gonzalez-Rodriguez, D.; Guillou, L.; Cornat, F.; Lafaurie-Janvore, J.; Babataheri, A.; de Langre, E.; Barakat, A. I.; Husson, J. Mechanical Criterion for the Rupture of a Cell Membrane under Compression. Biophys. J. 2016, 111, 2711-2721.

(31) Capozza, R.; Caprettini, V.; Gonano, C. A.; Bosca, A.; Moia, F.; Santoro, F.; De Angelis, F. Cell Membrane Disruption by Vertical Micro-/Nanopillars: Role of Membrane Bending and Traction Forces. ACS Appl. Mater. Interfaces 2018, 10, 29107-29114.

(32) Atkins, A. G.; Mai, Y. W. Elastic and Plastic Fracture: Metals, Polymers, Ceramics, Composites, Biological Materials; Ellis Horwood, 1985.

(33) Liu, Z.; Wang, C.; Chen, Z.; Sui, J. The advance of surgical blades in cutting soft biological tissue: a review. Int. $J$. Adv. Manuf. Technol. 2021, 113, 1817-1832.

(34) Doran, C. F.; McCormack, B. A. O.; Macey, A. A Simplified Model to Determine the Contribution of Strain Energy in the Failure Process of Thin Biological Membranes during Cutting. Strain 2008, 40, 173-179.

(35) Azar, T.; Hayward, V. Estimation of the Fracture Toughness of Soft Tissue from Needle Insertion. In Biomedical Simulation; Bello, F.; Edwards, P. J. E., Eds.; Springer Berlin Heidelberg: Berlin, Heidelberg, 2008; pp. 166-175. Gokgol, C.; Basdogan, C.; Canadinc, D. Estimation of fracture toughness of liver tissue: experiments and validation. Med Eng Phys 2012, 34, 882-891.

(37) Hu, Z.; Zhang, B.; Sun, W. Cutting characteristics of biological soft tissues. CIRP Annals 2012, 61, 135-138. Pissarenko, A.; Yang, W.; Quan, H.; Poyer, B.; Williams, A.; Brown, K. A.; Meyers, M. A. The toughness of porcine skin: Quantitative measurements and microstructural 
characterization. J Mech Behav Biomed Mater 2020, 109, 103848 .

(39) Griffith, A. A. The phenomena of rupture and flow in solids. Philosophical Transactions of the Royal Society A: Mathematical, Physical and Engineering Sciences 1921, 221, 163-198.

(40) Lake, G. J.; Yeoh, O. H. Measurement of rubber cutting resistance in the absence of friction. Int. J. Fract. 1978, $14,509-526$.

(41) Creton, C.; Ciccotti, M. Fracture and adhesion of soft materials: a review. Rep. Prog. Phys. 2016, 79, 046601.

(42) Zhang, B.; Shiang, C. S.; Yang, S. J.; Hutchens, S. B. YShaped Cutting for the Systematic Characterization of Cutting and Tearing. Exp Mech 2019, 59, 1-13.

(43) McCarthy, C. T.; Hussey, M.; Gilchrist, M. D. On the sharpness of straight edge blades in cutting soft solids: Part I - indentation experiments. Eng Fract Mech 2007, $74,2205-2224$.

(44) Hu, Z.; Sun, W.; Zhang, B. Characterization of aortic tissue cutting process: experimental investigation using porcine ascending aorta. J Mech Behav Biomed Mater 2013, 18, 81-89.

(45) McCarthy, C. T.; Annaidh, A. N.; Gilchrist, M. D. On the sharpness of straight edge blades in cutting soft solids: Part II - Analysis of blade geometry. Eng Fract Mech 2010, 77, 437-451.

(46) scienceofsharp. A Comparison of Several Manufactured Blades https://scienceofsharp.com/2014/05/28/acomparison-of-several-manufactured-blades (accessed Jun 27, 2021).

(47) Rogers, J. A.; Nuzzo, R. G. Recent progress in soft lithography. Materials Today 2005, 8, 50-56.

(48) Barbot, A.; Wales, D.; Yeatman, E.; Yang, G. Microfluidics at fiber tip for nanoliter delivery and sampling. $A d v$. Sci. 2021, 2004643.

(49) Alsharhan, A. T.; Acevedo, R.; Warren, R.; Sochol, R. D. 3D microfluidics via cyclic olefin polymer-based in situ direct laser writing. Lab Chip 2019, 19, 2799-2810.

(50) Wang, Z.; Volinsky, A. A.; Gallant, N. D. Crosslinking effect on polydimethylsiloxane elastic modulus measured by custom-built compression instrument. J. Appl. Polym. Sci. 2014, 131, n/a-n/a.

(51) Johnston, I. D.; McCluskey, D. K.; Tan, C. K. L.; Tracey, M. C. Mechanical characterization of bulk Sylgard 184 for microfluidics and microengineering. J. Micromech. Microeng. 2014, 24, 035017.

(52) Guimarães, C. F.; Gasperini, L.; Marques, A. P.; Reis, R. L. The stiffness of living tissues and its implications for tissue engineering. Nat. Rev. Mater. 2020.

(53) Sim, J. H.; Moon, H. J.; Roh, Y. H.; Jung, H. W.; Bong, K. W. Fabrication of NOA microfluidic devices based on sequential replica molding. Korean J. Chem. Eng. 2017, 34, 1495-1499.

(54) Iliescu, C.; Taylor, H.; Avram, M.; Miao, J.; Franssila, S. A practical guide for the fabrication of microfluidic devices using glass and silicon. Biomicrofluidics 2012, 6, $16505-1650516$

(55) Ben-Yakar, A.; Bourgeois, F. Ultrafast laser nanosurgery in microfluidics for genome-wide screenings. Curr. Opin. Biotechnol. 2009, 20, 100-105.

(56) Vogel, A.; Venugopalan, V. Mechanisms of pulsed laser ablation of biological tissues. Chem. Rev. 2003, 103, 577644.

(57) Niemz, M. H. Laser-Tissue Interactions: Fundamentals and Applications; Springer International Publishing: Cham, 2019.

(58) Zeigler, M. B.; Chiu, D. T. Laser selection significantly affects cell viability following single-cell nanosurgery. Photochem. Photobiol. 2009, 85, 1218-1224.

(59) Nishimura, N.; Schaffer, C. B.; Kleinfeld, D. In vivo manipulation of biological systems with femtosecond laser pulses. In High-Power Laser Ablation VI; Phipps, C. R., Ed.; SPIE Proceedings; SPIE, 2006; Vol. 6261, p. 62611J.

(60) Shen, N.; Schaffer, C. B.; Datta, D.; Mazur, E. Photodisruption in biological tissues and single cells using femtosecond laser pulses. In Technical Digest. Summaries of papers presented at the Conference on Lasers and Electro-Optics. Postconference Technical Digest (IEEE Cat. No.01CH37170); IEEE, 2001; pp. 403-404.

(61) Guo, S. X.; Bourgeois, F.; Chokshi, T.; Durr, N. J.; Hilliard, M. A.; Chronis, N.; Ben-Yakar, A. Femtosecond laser nanoaxotomy lab-on-a-chip for in vivo nerve regeneration studies. Nat. Methods 2008, 5, 531-533.

(62) Chung, K.; Lu, H. Automated high-throughput cell microsurgery on-chip. Lab Chip 2009, 9, 2764-2766.

(63) Samara, C.; Rohde, C. B.; Gilleland, C. L.; Norton, S.; Haggarty, S. J.; Yanik, M. F. Large-scale in vivo femtosecond laser neurosurgery screen reveals small-molecule enhancer of regeneration. Proc. Natl. Acad. Sci. USA 2010, 107, 18342-18347.

(64) Gokce, S. K.; Guo, S. X.; Ghorashian, N.; Everett, W. N.; Jarrell, T.; Kottek, A.; Bovik, A. C.; Ben-Yakar, A. A fully automated microfluidic femtosecond laser axotomy platform for nerve regeneration studies in C. elegans. PLoS One 2014, 9, e113917.

(65) Gokce, S. K.; Hegarty, E. M.; Mondal, S.; Zhao, P.; Ghorashian, N.; Hilliard, M. A.; Ben-Yakar, A. A multitrap microfluidic chip enabling longitudinal studies of nerve regeneration in Caenorhabditis elegans. Sci. Rep. 2017, 7, 9837 .

(66) Kim, Y.; Karthikeyan, K.; Chirvi, S.; Davé, D. P. Neurooptical microfluidic platform to study injury and regeneration of single axons. Lab Chip 2009, 9, 2576-2581.

(67) Niioka, H.; Smith, N. I.; Fujita, K.; Inouye, Y.; Kawata, S. Femtosecond laser nano-ablation in fixed and non-fixed cultured cells. Opt. Express 2008, 16, 14476-14495.

(68) Ghannad-Rezaie, M.; Wang, X.; Mishra, B.; Collins, C.; Chronis, N. Microfluidic chips for in vivo imaging of cellular responses to neural injury in Drosophila larvae. PLoS One 2012, 7, e29869.

(69) Zeigler, M. B.; Chiu, D. T. Single-cell nanosurgery. Methods Mol. Biol. 2013, 991, 139-148.

(70) Laser Precision Microfabrication; Sugioka, K.; Meunier, M.; Piqué, A., Eds.; Springer series in materials science; Springer Berlin Heidelberg: Berlin, Heidelberg, 2010; Vol. 135.

(71) Vogel, A.; Noack, J.; Hüttman, G.; Paltauf, G. Mechanisms of femtosecond laser nanosurgery of cells and tissues. Appl. Phys. B 2005, 81, 1015-1047.

(72) Compton, J. L.; Hellman, A. N.; Venugopalan, V. Hydrodynamic determinants of cell necrosis and molecular delivery produced by pulsed laser microbeam irradiation of adherent cells. Biophys. J. 2013, 105, 2221-2231.

(73) Cai, D. K.; Neyer, A.; Kuckuk, R.; Heise, H. M. Optical absorption in transparent PDMS materials applied for multimode waveguides fabrication. Opt Mater (Amst) 2008, 30, 1157-1161.

(74) Garavito, R. M.; Ferguson-Miller, S. Detergents as tools in membrane biochemistry. J. Biol. Chem. 2001, 276, 32403-32406.

(75) Heerklotz, H. Interactions of surfactants with lipid membranes. Q Rev Biophys 2008, 41, 205-264.

(76) Dourmashkin, R. R.; Dougherty, R. M.; Harris, R. J. Electron microscopic observations on Rous sarcoma virus and cell membranes. Nature 1962, 194, 1116-1119.

(77) Lichtenberg, D.; Ahyayauch, H.; Alonso, A.; Goñi, F. M. Detergent solubilization of lipid bilayers: a balance of driving forces. Trends Biochem. Sci. 2013, 38, 85-93.

(78) Lichtenberg, D.; Ahyayauch, H.; Goñi, F. M. The mechanism of detergent solubilization of lipid bilayers. Biophys. J. 2013, 105, 289-299. 
(79) Nazari, M.; Kurdi, M.; Heerklotz, H. Classifying surfactants with respect to their effect on lipid membrane order. Biophys. J. 2012, 102, 498-506.

(80) Windaus, A. Über die Entgiftung der Saponine durch Cholesterin. Ber. Dtsch. Chem. Ges. 1909, 42, 238-246.

(101) Kozlov, M. M.; Markin, V. S. [Possible mechanism of membrane fusion]. Biofizika 1983, 28, 242-247.

(102) Markin, V. S.; Kozlov, M. M.; Borovjagin, V. L. On the theory of membrane fusion. The stalk mechanism. Gen Physiol Biophys 1984, 3, 361-377.

(103) Markin, V. S.; Albanesi, J. P. Membrane fusion: stalk model revisited. Biophys. J. 2002, 82, 693-712.
(104) White, J. M. Membrane fusion. Science 1992, 258, 917924.

(105) Chiu, F. W. Y.; Bagci, H.; Fisher, A. G.; deMello, A. J.; Elvira, K. S. A microfluidic toolbox for cell fusion. $J$. Chem. Technol. Biotechnol. 2016, 91, 16-24.

(106) Duelli, D.; Lazebnik, Y. Cell-to-cell fusion as a link between viruses and cancer. Nat. Rev. Cancer 2007, 7, 968976.

(107) Köhler, G.; Milstein, C. Continuous cultures of fused cells secreting antibody of predefined specificity. Nature 1975, 256, 495-497.

(108) Leroy, H.; Han, M.; Woottum, M.; Bracq, L.; Bouchet, J.; Xie, M.; Benichou, S. Virus-Mediated Cell-Cell Fusion. Int. J. Mol. Sci. 2020, 21.

(109) Chen, E. H.; Olson, E. N. Unveiling the mechanisms of cell-cell fusion. Science 2005, 308, 369-373.

(110) Knutton, S. The mechanism of virus-induced cell fusion Micron (1969) 1978, 9, 133-154.

(111) Okada, Y. [3] Sendai virus-induced cell fusion. In Membrane fusion techniques part B; Methods in Enzymology; Elsevier, 1993; Vol. 221, pp. 18-41.

(112) Okada, Y. Chapter 10 Sendai Virus-Mediated Cell Fusion. In Membrane fusion in fertilization, cellular transport, and viral infection; Current topics in membranes and transport; Elsevier, 1988; Vol. 32, pp. 297-336.

(113) Yang, J.; Shen, M. H. Polyethylene glycol-mediated cell fusion. Methods Mol. Biol. 2006, 325, 59-66.

(114) Ahkong, Q. F.; Fisher, D.; Tampion, W.; Lucy, J. A. Mechanisms of cell fusion. Nature 1975, 253, 194-195.

(115) Davidson, R. L.; Gerald, P. S. Improved techniques for the induction of mammalian cell hybridization by polyethylene glycol. Somatic Cell Genet. 1976, 2, 165-176.

(116) Lentz, B. R.; Lee, J. K. Poly(ethylene glycol) (PEG)mediated fusion between pure lipid bilayers: a mechanism in common with viral fusion and secretory vesicle release? Mol Membr Biol 1999, 16, 279-296.

(117) MacDonald, R. I. Membrane fusion due to dehydration by polyethylene glycol, dextran, or sucrose. Biochemistry 1985, 24, 4058-4066.

(118) Hui, S. W.; Kuhl, T. L.; Guo, Y. Q.; Israelachvili, J. Use of poly(ethylene glycol) to control cell aggregation and fusion. Colloids and Surfaces B: Biointerfaces 1999, 14, 213-222.

(119) Blow, A. M.; Botham, G. M.; Fisher, D.; Goodall, A. H.; Tilcock, C. P.; Lucy, J. A. Water and calcium ions in cell fusion induced by poly(ethylene glycol). FEBS Lett. 1978, 94, 305-310.

(120) Matter, F.; Luna, A. L.; Niederberger, M. From colloidal dispersions to aerogels: How to master nanoparticle gelation. Nano Today 2020, 30, 100827.

(121) Lee, J.; Lentz, B. R. Outer leaflet-packing defects promote poly(ethylene glycol)-mediated fusion of large unilamellar vesicles. Biochemistry 1997, 36, 421-431.

(122) Lehtonen, J. Y.; Kinnunen, P. K. Changes in the lipid dynamics of liposomal membranes induced by poly(ethylene glycol): free volume alterations revealed by inter- and intramolecular excimer-forming phospholipid analogs. Biophys. J. 1994, 66, 1981-1990.

(123) Lentz, B. R. Polymer-induced membrane fusion: potential mechanism and relation to cell fusion events. Chem Phys Lipids 1994, 73, 91-106.

(124) Honda, K.; Maeda, Y.; Sasakawa, S.; Ohno, H.; Tsuchida, E. The components contained in polyethylene glycol of commercial grade (PEG-6,000) as cell fusogen. Biochem. Biophys. Res. Commun. 1981, 101, 165-171.

(125) Davidson, R. L.; Gerald, P. S. Chapter 18 induction of mammalian somatic cell hybridization by polyethylene glycol. In Methods in cell biology volume 15; Methods in cell biology; Elsevier, 1977; Vol. 15, pp. 325-338.

(126) Roos, D. S.; Davidson, R. L. Isolation of mouse cell lines resistant to the fusion-inducing effect of polyethylene glycol. Somatic Cell Genet. 1980, 6, 381-390. 
(127) Hoffman, A. N.; Bamba, R.; Pollins, A. C.; Thayer, W. P. Analysis of polyethylene glycol (PEG) fusion in cultured neuroblastoma cells via flow cytometry: Techniques \& optimization. J. Clin. Neurosci. 2017, 36, 125-128.

(128) Messineo, E.; Pollins, A.; Thayer, W. Optimization and evaluation of an in vitro model of PEG-mediated fusion of nerve cell bodies. J. Clin. Neurosci. 2019, 63, 189-195.

(129) Zimmermann, U.; Scheurich, P. High frequency fusion of plant protoplasts by electric fields. Planta 1981, 151, 2632.

(130) Hu, N.; Yang, J.; Joo, S. W.; Banerjee, A. N.; Qian, S. Cell electrofusion in microfluidic devices: A review. Sensors and Actuators B: Chemical 2013, 178, 63-85.

(131) Abidor, I. G.; Sowers, A. E. Kinetics and mechanism of cell membrane electrofusion. Biophys. J. 1992, 61, 15571569.

(132) Klöck, G.; Zimmermann, U. Facilitated electrofusion of vacuolated $\times$ evacuolated oat mesophyll protoplasts in hypo-osmolar media after alignment with an alternating field of modulated strength. Biochimica et Biophysica Acta (BBA) - Biomembranes 1990, 1025, 87-93.

(133) Wang, K.; Sun, X. H.; Zhang, Y.; Zhang, T.; Zheng, Y.; Wei, Y. C.; Zhao, P.; Chen, D. Y.; Wu, H. A.; Wang, W. $\mathrm{H}$.; et al. Characterization of cytoplasmic viscosity of hundreds of single tumour cells based on micropipette aspiration. R. Soc. Open Sci. 2019, 6, 181707.

(134) Verkman, A. S. Solute and macromolecule diffusion in cellular aqueous compartments. Trends Biochem. Sci. 2002, 27, 27-33.

(135) Ghosal, S. Fluid mechanics of electroosmotic flow and its effect on band broadening in capillary electrophoresis. Electrophoresis 2004, 25, 214-228.

(136) Anderson, J. L. Colloid transport by interfacial forces. Annu. Rev. Fluid Mech. 1989, 21, 61-99.

(137) O'Brien, R. W.; White, L. R. Electrophoretic mobility of a spherical colloidal particle. J. Chem. Soc., Faraday Trans. 2 1978, 74, 1607.

(138) Li, S. F. Y.; Wu, Y. S. Capillary electrophoresis. Electrophoresis 2000, 1176-1187.

(139) Yeh, I.-C.; Hummer, G. Diffusion and electrophoretic mobility of single-stranded RNA from molecular dynamics simulations. Biophys. J. 2004, 86, 681-689.

(140) Pohl, H. A.; Crane, J. S. Dielectrophoresis of cells. Biophys. J. 1971, 11, 711-727.

(141) Castellanos, A.; Ramos, A.; González, A.; Green, N. G.; Morgan, H. Electrohydrodynamics and dielectrophoresis in microsystems: scaling laws. J. Phys. D, Appl. Phys. 2003, 36, 2584-2597.

(142) Freedman, K. J.; Otto, L. M.; Ivanov, A. P.; Barik, A.; Oh, S.-H.; Edel, J. B. Nanopore sensing at ultra-low concentrations using single-molecule dielectrophoretic trapping. Nat. Commun. 2016, 7, 10217.

(143) Nadappuram, B. P.; Cadinu, P.; Barik, A.; Ainscough, A. J.; Devine, M. J.; Kang, M.; Gonzalez-Garcia, J.; Kittler, J. T.; Willison, K. R.; Vilar, R.; et al. Nanoscale tweezers for single-cell biopsies. Nat. Nanotechnol. 2019, 14, 80 88.

(144) Gruner, G. Conductivity and dielectric constant of the DNA double helix. In AIP Conference Proceedings; AIP, 2000; pp. 462-467.

(145) Foster, K. R.; Bidinger, J. M.; Carpenter, D. O. The electrical resistivity of cytoplasm. Biophys. J. 1976, 16, 9911001.

(146) Mugele, F.; Baret, J.-C. Electrowetting: from basics to applications. J. Phys.: Condens. Matter 2005, 17, R705R774.

(147) Liu, Y.; Yang, M.; Deng, Y.; Su, G.; Enninful, A.; Guo, C. C.; Tebaldi, T.; Zhang, D.; Kim, D.; Bai, Z.; et al. High-Spatial-Resolution Multi-Omics Sequencing via Deterministic Barcoding in Tissue. Cell 2020, 183, 16651681.e18.
(148) Rodriguez, A. D.; Horowitz, L. F.; Castro, K.; Kenerson, H.; Bhattacharjee, N.; Gandhe, G.; Raman, A.; Monnat, R. J.; Yeung, R.; Rostomily, R. C.; et al. A microfluidic platform for functional testing of cancer drugs on intact tumor slices. Lab Chip 2020, 20, 1658-1675.

(149) Horowitz, L. F.; Rodriguez, A. D.; Dereli-Korkut, Z.; Lin, R.; Castro, K.; Mikheev, A. M.; Monnat, R. J.; Folch, A.; Rostomily, R. C. Multiplexed drug testing of tumor slices using a microfluidic platform. NPJ Precis. Oncol. 2020, 4,12 .

(150) Horowitz, L. F.; Rodriguez, A. D.; Au-Yeung, A.; Bishop, K. W.; Barner, L. A.; Mishra, G.; Raman, A.; Delgado, P.; Liu, J. T. C.; Gujral, T. S.; et al. Microdissected "cuboids" for microfluidic drug testing of intact tissues. Lab Chip 2020.

(151) Gouveia, C.; Huyser, C.; Egli, D.; Pepper, M. S. Lessons Learned from Somatic Cell Nuclear Transfer. Int. J. Mol. Sci. 2020, 21.

(152) Iuso, D.; Czernik, M.; Zacchini, F.; Ptak, G.; Loi, P. A simplified approach for oocyte enucleation in mammalian cloning. Cell. Reprogram. 2013, 15, 490-494.

(153) Zhao, X.; Cui, M.; Zhang, Y.; Liu, Y.; Zhao, X. Robotic precisely oocyte blind enucleation method. Appl. Sci. 2021, $11,1850$.

(154) Haibo Huang; Dong Sun; Mills, J. K.; Li, W. J.; Shuk Han Cheng. Visual-Based Impedance Control of Out-of-Plane Cell Injection Systems. IEEE Trans. Automat. Sci. Eng. 2009, 6, 565-571.

(155) Yang, H.; Li, X.; Liu, Y.; Sun, D. Automated transportation of biological cells for multiple processing steps in cell surgery. IEEE Trans. Automat. Sci. Eng. 2017, 14, 1712-1721.

(156) Hagiwara, M.; Ichikawa, A.; Kawahara, T.; Arai, F. High speed enucleation of oocyte using magnetically actuated microrobot on a chip. In 20127 th IEEE International Conference on Nano/Micro Engineered and Molecular Systems (NEMS); IEEE, 2012; pp. 364-367.

(157) Hagiwara, M.; Kawahara, T.; Yamanishi, Y.; Masuda, T.; Feng, L.; Arai, F. On-chip magnetically actuated robot with ultrasonic vibration for single cell manipulations. Lab Chip 2011, 11, 2049-2054.

(158) Feng, L.; Hagiwara, M.; Ichikawa, A.; Arai, F. On-Chip Enucleation of Bovine Oocytes using' 'MicrorobotAssisted Flow-Speed Control. Micromachines (Basel) 2013, 4, 272-285.

(159) Ichikawa, A.; Tanikawa, T.; Matsukawa, K.; Takahashi, S.; Ohba, K. Fluorescent monitoring using microfluidics chip and development of syringe pump for automation of enucleation to automate cloning. In 2009 IEEE International Conference on Robotics and Automation; IEEE, 2009; pp. 2231-2236.

(160) Ichikawa, A.; Takahashi, S.; Matsukawa, K.; Tanikawa, T.; Ohba, K. Injection and cutting methods of animal cells using a microfluidic chip. In 2008 IEEE International Conference on Robotics and Automation; IEEE, 2008; pp. 868-873.

(161) Ichikawa, A.; Tanikawa, T.; Matsukawa, K.; Takahashi, S.; Ohba, K. Automated Cell-Cutting for Cell Cloning. SICE JCMSI 2010, 3, 75-80.

(162) Regeneration in Protozoa: A Problem of Morphogenesis on JSTOR https://www.jstor.org/stable/2808978\#metadata_info_tab_ contents (accessed May 10, 2021).

(163) Tartar, V. The Biology of Stentor; International Series of Monographs on Pure and Applied Biology; Pergammon, 1961; Vol. 5.

(164) Schmidt, H. D. On the Minute Structure of the Hepatic Lobules, particularly with reference to the Relationship between the Capillary Bloodvessels, the Hepatic Cells, and the Canals which carry off the Secretion of the latter. Am. J. Med. Sci. 1859, 37. 
(165) Ozil, J. P. Production of identical twins by bisection of blastocysts in the cow. J. Reprod. Fertil. 1983, 69, 463468.

(166) Slabodnick, M.; Prevo, B.; Gross, P.; Sheung, J.; Marshall, W. Visualizing cytoplasmic flow during single-cell wound healing in Stentor coeruleus. J. Vis. Exp. 2013, e50848.

(167) Koppaka, S.; Blauch, L. R.; Zhang, K. S.; Cordts, S.; Tang, S. K. Y. Fabrication and characterization of 3D microfluidics blades to improve the cutting of biological materials. In; Chemical and Biological Microsystems Society, 2020; pp. T3-316.b.

(168) Paul, R.; Zhang, K. S.; Tang, S. K. Y. Hydrodynamic Cell Splitter as a Microfluidic Tool to Study Single-cell Wound Healing and Regeneration. In; American Society of Cell Biology \& European Molecular Biology Organization, 2020; p. 101.

(169) Wyckoff, J. B.; Jones, J. G.; Condeelis, J. S.; Segall, J. E. A critical step in metastasis: in vivo analysis of intravasation at the primary tumor. Cancer Res. 2000, 60, 25042511.

(170) Weiss, L.; Nannmark, U.; Johansson, B. R.; Bagge, U. Lethal deformation of cancer cells in the microcirculation: a potential rate regulator of hematogenous metastasis. Int. J. Cancer 1992, 50, 103-107.

(171) Kamyabi, N.; Vanapalli, S. A. Microfluidic cell fragmentation for mechanical phenotyping of cancer cells. Biomicrofluidics 2016, 10, 021102.

(172) López-Otín, C.; Blasco, M. A.; Partridge, L.; Serrano, M.; Kroemer, G. The hallmarks of aging. Cell 2013, 153, 1194-1217.

(173) Hanahan, D.; Weinberg, R. A. Hallmarks of cancer: the next generation. Cell 2011, 144, 646-674.

(174) Maskell, D.; Kennedy, A.; Hodgson, J.; Smart, K. Chronological and replicative lifespan of polyploid (syn. ). FEMS Yeast Res 2003, 3, 201-209.

(175) Steffen, K. K.; Kennedy, B. K.; Kaeberlein, M. Measuring replicative life span in the budding yeast. J. Vis. Exp. 2009.

(176) Ryley, J.; Pereira-Smith, O. M. Microfluidics device for single cell gene expression analysis in Saccharomyces cerevisiae. Yeast 2006, 23, 1065-1073.

(177) Lee, S. S.; Avalos Vizcarra, I.; Huberts, D. H. E. W.; Lee, L. P.; Heinemann, M. Whole lifespan microscopic observation of budding yeast aging through a microfluidic dissection platform. Proc. Natl. Acad. Sci. USA 2012, 109, 4916-4920.

(178) Zhang, Y.; Luo, C.; Zou, K.; Xie, Z.; Brandman, O.; Ouyang, Q.; Li, H. Single cell analysis of yeast replicative aging using a new generation of microfluidic device. PLoS One 2012, 7, e48275.

(179) Crane, M. M.; Clark, I. B. N.; Bakker, E.; Smith, S.; Swain, P. S. A microfluidic system for studying ageing and dynamic single-cell responses in budding yeast. PLoS One 2014, 9, e100042.

(180) Jo, M. C.; Liu, W.; Gu, L.; Dang, W.; Qin, L. Highthroughput analysis of yeast replicative aging using a microfluidic system. Proc. Natl. Acad. Sci. USA 2015, 112, 9364-9369.

(181) Altschuler, S. J.; Wu, L. F. Cellular heterogeneity: do differences make a difference? Cell 2010, 141, 559-563.

(182) Dagogo-Jack, I.; Shaw, A. T. Tumour heterogeneity and resistance to cancer therapies. Nat. Rev. Clin. Oncol. 2018, 15, 81-94.

(183) Qiu, X.; Huang, J.-H.; Westerhof, T. M.; Lombardo, J. A.; Henrikson, K. M.; Pennell, M.; Pourfard, P. P.; Nelson, E. L.; Nath, P.; Haun, J. B. Microfluidic channel optimization to improve hydrodynamic dissociation of cell aggregates and tissue. Sci. Rep. 2018, 8, 2774.

(184) Reichard, A.; Asosingh, K. Best Practices for Preparing a Single Cell Suspension from Solid Tissues for Flow Cytometry. Cytometry A 2019, 95, 219-226.
(185) Lin, C.-H.; Lee, D.-C.; Chang, H.-C.; Chiu, I.-M.; Hsu, C.-H. Single-cell enzyme-free dissociation of neurospheres using a microfluidic chip. Anal. Chem. 2013, 85, 11920-11928.

(186) Qiu, X.; Lombardo, J. A.; Westerhof, T. M.; Pennell, M.; Ng, A.; Alshetaiwi, H.; Luna, B. M.; Nelson, E. L.; Kessenbrock, K.; Hui, E. E.; et al. Microfluidic filter device with nylon mesh membranes efficiently dissociates cell aggregates and digested tissue into single cells. $L a b$ Chip 2018, 18, 2776-2786.

(187) Qiu, X.; De Jesus, J.; Pennell, M.; Troiani, M.; Haun, J. B. Microfluidic device for mechanical dissociation of cancer cell aggregates into single cells. Lab Chip 2015, 15, 339350 .

(188) Lombardo, J. A.; Aliaghaei, M.; Nguyen, Q. H.; Kessenbrock, K.; Haun, J. B. Microfluidic platform accelerates tissue processing into single cells for molecular analysis and primary culture models. Nat. Commun. 2021, $12,2858$.

(189) Wallman, L.; Åkesson, E.; Ceric, D.; Andersson, P. H.; Day, K.; Hovatta, O.; Falci, S.; Laurell, T.; Sundström, E. Biogrid--a microfluidic device for large-scale enzymefree dissociation of stem cell aggregates. Lab Chip 2011, 11, 3241-3248.

(190) Reddien, P. W. The cellular and molecular basis for planarian regeneration. Cell 2018, 175, 327-345.

(191) Toda, S.; Blauch, L. R.; Tang, S. K. Y.; Morsut, L.; Lim, W. A. Programming self-organizing multicellular structures with synthetic cell-cell signaling. Science 2018, 361, 156-162.

(192) Hopcroft, M. A.; Nix, W. D.; Kenny, T. W. What is the young's modulus of silicon? J. Microelectromech. Syst. 2010, 19, 229-238.

(193) Ganguli, A.; Ornob, A.; Spegazzini, N.; Liu, Y.; Damhorst, G.; Ghonge, T.; Thornton, B.; Konopka, C. J.; Dobrucki, W.; Clare, S. E.; et al. Pixelated spatial gene expression analysis from tissue. Nat. Commun. 2018, 9, 202.

(194) Cordts, S. C.; Castaño, N.; Koppaka, S.; Tang, S. K. Y. Fabrication of a silicon $\mu$ Dicer for uniform microdissection of tissue samples. Appl. Phys. Lett. 2021, 119, 011904.

(195) Lemma, E. D.; Rizzi, F.; Dattoma, T.; Spagnolo, B.; Sileo, L.; Qualtieri, A.; De Vittorio, M.; Pisanello, F. Mechanical properties tunability of three-dimensional polymeric structures in two-photon lithography. IEEE Trans. Nanotechnol. 2016, 1-1.

(196) Thomas, C. R.; Zhang, Z. The effect of hydrodynamics on biological materials. In Advances in bioprocess engineering; Galindo, E.; Ramírez, O. T., Eds.; Springer Netherlands: Dordrecht, 1998; pp. 137-170.

(197) Kohles, S. S.; Nève, N.; Zimmerman, J. D.; Tretheway, D. C. Mechanical stress analysis of microfluidic environments designed for isolated biological cell investigations. J Biomech Eng 2009, 131, 121006.

(198) Belen, D.; Aciduman, A.; Er, U. History of peripheral nerve repair: may the procedure have been practiced in Hippocratic School? Surg Neurol 2009, 72, 190-3; discussion 193.

(199) West, J. R. Early history of mammalian nerve regeneration. Neuroscience \& Biobehavioral Reviews 1978, 2, 2732.

(200) Nissl, F. Über die Veränderungen der Ganglienzellen am Facialiskern des Kaninchens nach Ausreissung der Nerven. Allg. Z. Psychiat. Are Grenzgeb 1892.

(201) Grafstein, B. The nerve cell body response to axotomy. Exp. Neurol. 1975, 48, 32-51.

(202) DeFelipe, J.; Jones, E. G. Cajal's degeneration and regeneration of the nervous system; Oxford University Press: New York, 1928

(203) Siddique, R.; Thakor, N. Investigation of nerve injury through microfluidic devices. J. R. Soc. Interface 2014, 11,20130676 
(204) Shrirao, A. B.; Kung, F. H.; Omelchenko, A.; Schloss, R. S.; Boustany, N. N.; Zahn, J. D.; Yarmush, M. L.; Firestein, B. L. Microfluidic platforms for the study of neuronal injury in vitro. Biotechnol. Bioeng. 2018, 115, $815-830$

(205) Taylor, A. M.; Rhee, S. W.; Tu, C. H.; Cribbs, D. H.; Cotman, C. W.; Jeon, N. L. Microfluidic multicompartment device for neuroscience research. Langmuir $\mathbf{2 0 0 3}$ $19,1551-1556$.

(206) Taylor, A. M.; Blurton-Jones, M.; Rhee, S. W.; Cribbs, D. H.; Cotman, C. W.; Jeon, N. L. A microfluidic culture platform for CNS axonal injury, regeneration and transport. Nat. Methods 2005, 2, 599-605.

(207) Tong, Z.; Segura-Feliu, M.; Seira, O.; Homs-Corbera, A.; Del Río, J. A.; Samitier, J. A microfluidic neuronal platform for neuron axotomy and controlled regenerative studies. RSC Adv. 2015, 5, 73457-73466.

(208) Park, J. W.; Vahidi, B.; Taylor, A. M.; Rhee, S. W.; Jeon, N. L. Microfluidic culture platform for neuroscience research. Nat. Protoc. 2006, 1, 2128-2136.

(209) Taylor, A. M.; Berchtold, N. C.; Perreau, V. M.; Tu, C. H.; Li Jeon, N.; Cotman, C. W. Axonal mRNA in uninjured and regenerating cortical mammalian axons. J. Neurosci. 2009, 29, 4697-4707.

(210) Zhang, J.-N.; Michel, U.; Lenz, C.; Friedel, C. C.; Köster, S.; d Hedouville, Z.; Tönges, L.; Urlaub, H.; Bähr, M.; Lingor, P.; et al. Calpain-mediated cleavage of collapsin response mediator protein-2 drives acute axonal degeneration. Sci. Rep. 2016, 6, 37050.

(211) Kim, H. J.; Park, J. W.; Byun, J. H.; Vahidi, B.; Rhee, S. W.; Jeon, N. L. Integrated microfluidics platforms for investigating injury and regeneration of CNS axons. Ann. Biomed. Eng. 2012, 40, 1268-1276.

(212) Nagendran, T.; Larsen, R. S.; Bigler, R. L.; Frost, S. B.; Philpot, B. D.; Nudo, R. J.; Taylor, A. M. Distal axotomy enhances retrograde presynaptic excitability onto injured pyramidal neurons via trans-synaptic signaling. Nat. Commun. 2017, 8, 625.

(213) Holland, S. M.; Collura, K. M.; Ketschek, A.; Noma, K.; Ferguson, T. A.; Jin, Y.; Gallo, G.; Thomas, G. M. Palmitoylation controls DLK localization, interactions and activity to ensure effective axonal injury signaling. Proc. Natl. Acad. Sci. USA 2016, 113, 763-768.

(214) Kilinc, D.; Peyrin, J.-M.; Soubeyre, V.; Magnifico, S.; Saias, L.; Viovy, J.-L.; Brugg, B. Wallerian-like degeneration of central neurons after synchronized and geometrically registered mass axotomy in a three-compartmental microfluidic chip. Neurotox Res 2011, 19, 149-161.

(215) Lee, C. Y.; Romanova, E. V.; Sweedler, J. V. Laminar stream of detergents for subcellular neurite damage in a microfluidic device: a simple tool for the study of neuroregeneration. J. Neural Eng. 2013, 10, 036020.

(216) Deleglise, B.; Lassus, B.; Soubeyre, V.; AlleaumeButaux, A.; Hjorth, J. J.; Vignes, M.; Schneider, B.; Brugg, B.; Viovy, J.-L.; Peyrin, J.-M. Synapto-protective drugs evaluation in reconstructed neuronal network. PLoS One 2013, 8, e71103.

(217) Deleglise, B.; Lassus, B.; Soubeyre, V.; Doulazmi, M.; Brugg, B.; Vanhoutte, P.; Peyrin, J.-M. Dysregulated Neurotransmission induces Trans-synaptic degeneration in reconstructed Neuronal Networks. Sci. Rep. 2018, 8, 11596.

(218) Hosmane, S.; Fournier, A.; Wright, R.; Rajbhandari, L.; Siddique, R.; Yang, I. H.; Ramesh, K. T.; Venkatesan, A.; Thakor, N. Valve-based microfluidic compression platform: single axon injury and regrowth. Lab Chip 2011, 11, 3888-3895.

(219) Magidson, V.; Loncarek, J.; Hergert, P.; Rieder, C. L.; Khodjakov, A. Laser microsurgery in the GFP era: A cell biologist's perspective. Laser Manipulation of Cells and Tissues 2007, 82, 239-266.
(220) Stirman, J. N.; Harker, B.; Lu, H.; Crane, M. M. Animal microsurgery using microfluidics. Curr. Opin. Biotechnol. 2014, 25, 24-29.

(221) Hellman, A. N.; Vahidi, B.; Kim, H. J.; Mismar, W.; Steward, O.; Jeon, N. L.; Venugopalan, V. Examination of axonal injury and regeneration in micropatterned neuronal culture using pulsed laser microbeam dissection. Lab Chip 2010, 10, 2083-2092.

(222) Aumeier, C.; Schaedel, L.; Gaillard, J.; John, K.; Blanchoin, L.; Théry, M. Self-repair promotes microtubule rescue. Nat. Cell Biol. 2016, 18, 1054-1064.

(223) Walker, R. A.; Inoué, S.; Salmon, E. D. Asymmetric behavior of severed microtubule ends after ultravioletmicrobeam irradiation of individual microtubules in vitro. J. Cell Biol. 1989, 108, 931-937.

(224) Schaedel, L.; John, K.; Gaillard, J.; Nachury, M. V.; Blanchoin, L.; Théry, M. Microtubules self-repair in response to mechanical stress. Nat. Mater. 2015, 14, 11561163.

(225) Todaro, G. J.; Lazar, G. K.; Green, H. The initiation of cell division in a contact-inhibited mammalian cell line. $J$ Cell Comp Physiol 1965, 66, 325-333.

(226) Gurney, T. Local stimulation of growth in primary cultures of chick embryo fibroblasts. Proc. Natl. Acad. Sci. USA 1969, 62, 906-911.

(227) Shabestani Monfared, G.; Ertl, P.; Rothbauer, M. Microfluidic and Lab-on-a-Chip Systems for Cutaneous Wound Healing Studies. 2021.

(228) Sticker, D.; Lechner, S.; Jungreuthmayer, C.; Zanghellini, J.; Ertl, P. Microfluidic migration and wound healing assay based on mechanically induced injuries of defined and highly reproducible areas. Anal. Chem. 2017, 89, 23262333.

(229) Go, H.; Tian, T.; Rhee, S. W. Fabrication of microfluidic chip for investigation of wound healing processes. BioChip J. 2018, 12, 146-153.

(230) Zhang, M.; Li, H.; Ma, H.; Qin, J. A simple microfluidic strategy for cell migration assay in an in vitro woundhealing model. Wound Repair Regen. 2013, 21, 897-903.

(231) Nikolić, D. L.; Boettiger, A. N.; Bar-Sagi, D.; Carbeck, J. D.; Shvartsman, S. Y. Role of boundary conditions in an experimental model of epithelial wound healing. Am. J. Physiol. Cell Physiol. 2006, 291, C68-75.

(232) Poujade, M.; Grasland-Mongrain, E.; Hertzog, A.; Jouanneau, J.; Chavrier, P.; Ladoux, B.; Buguin, A.; Silberzan, P. Collective migration of an epithelial monolayer in response to a model wound. Proc. Natl. Acad. Sci. USA 2007, 104, 15988-15993.

(233) Kim, H. N.; Hong, Y.; Kim, M. S.; Kim, S. M.; Suh, K.$\mathrm{Y}$. Effect of orientation and density of nanotopography in dermal wound healing. Biomaterials 2012, 33, 87828792 .

(234) Wang, Y.; Zhu, J.; Chen, P.; Li, Y.; Yan, S.; Wang, J.; Du, W.; Liu, B.-F. Wound-on-a-chip: High-throughput 3D wound healing assay with a novel SU-8 mesh chip. Sensors and Actuators B: Chemical 2019, 280, 86-93.

(235) Wei, Y.; Chen, F.; Zhang, T.; Chen, D.; Jia, X.; Tong, J.; Wang, J.; Guo, W.; Chen, J. A tubing-free microfluidic wound-healing assay quantifying vascular smooth muscle cell migration. In 2015 Transducers - 2015 18th International Conference on Solid-State Sensors, Actuators and Microsystems (TRANSDUCERS); IEEE, 2015; pp. 17781781.

(236) Conde, A. J.; Salvatierra, E.; Podhajcer, O.; Fraigi, L.; Madrid, R. E. Wound healing assay in a low-cost microfluidic platform. J. Phys.: Conf. Ser. 2013, 477, 012035.

(237) van der Meer, A. D.; Vermeul, K.; Poot, A. A.; Feijen, J.; Vermes, I. A microfluidic wound-healing assay for quantifying endothelial cell migration. Am. J. Physiol. Heart Circ. Physiol. 2010, 298, H719-25.

(238) Lin, J.-Y.; Lo, K.-Y.; Sun, Y.-S. A microfluidics-based wound-healing assay for studying the effects of shear 
stresses, wound widths, and chemicals on the woundhealing process. Sci. Rep. 2019, 9, 20016.

(239) Murrell, M.; Kamm, R.; Matsudaira, P. Tension, free space, and cell damage in a microfluidic wound healing assay. PLoS One 2011, 6, e24283.

(240) Felder, M.; Sallin, P.; Barbe, L.; Haenni, B.; Gazdhar, A.; Geiser, T.; Guenat, O. Microfluidic wound-healing assay to assess the regenerative effect of HGF on wounded alveolar epithelium. Lab Chip 2012, 12, 640-646.

(241) Nie, F.-Q.; Yamada, M.; Kobayashi, J.; Yamato, M.; Kikuchi, A.; Okano, T. On-chip cell migration assay using microfluidic channels. Biomaterials 2007, 28, 4017-4022.

(242) Lee, I.; Kim, D.; Park, G.-L.; Jeon, T.-J.; Kim, S. M. Investigation of wound healing process guided by nanoscale topographic patterns integrated within a microfluidic system. PLoS One 2018, 13, e0201418.

(243) Soitu, C.; Panea, M.; Castrejón-Pita, A. A.; Cook, P. R.; Walsh, E. J. Creating wounds in cell monolayers using micro-jets. Biomicrofluidics 2021, 15, 014108.

(244) Ben-Yakar, A.; Chronis, N.; Lu, H. Microfluidics for the analysis of behavior, nerve regeneration, and neural cell biology in C. elegans. Curr. Opin. Neurobiol. 2009, 19 , 561-567.

(245) Zeng, F.; Rohde, C. B.; Yanik, M. F. Sub-cellular precision on-chip small-animal immobilization, multi-photon imaging and femtosecond-laser manipulation. Lab Chip 2008, 8, 653-656.

(246) Rohde, C. B.; Zeng, F.; Gonzalez-Rubio, R.; Angel, M.; Yanik, M. F. Microfluidic system for on-chip highthroughput whole-animal sorting and screening at subcellular resolution. Proc. Natl. Acad. Sci. USA 2007, 104. 13891-13895.

(247) Chokshi, T. V.; Ben-Yakar, A.; Chronis, N. CO2 and compressive immobilization of C. elegans on-chip. Lab Chip 2009, 9, 151-157.

(248) Hulme, S. E.; Shevkoplyas, S. S.; Apfeld, J.; Fontana, W.; Whitesides, G. M. A microfabricated array of clamps for immobilizing and imaging C. elegans. Lab Chip 2007, 7, $1515-1523$.

(249) Pinan-Lucarre, B.; Gabel, C. V.; Reina, C. P.; Hulme, S. E.; Shevkoplyas, S. S.; Slone, R. D.; Xue, J.; Qiao, Y.; Weisberg, S.; Roodhouse, K.; et al. The core apoptotic executioner proteins CED-3 and CED-4 promote initiation of neuronal regeneration in Caenorhabditis elegans. PLoS Biol. 2012, 10, e1001331.

(250) Allen, P. B.; Sgro, A. E.; Chao, D. L.; Doepker, B. E.; Scott Edgar, J.; Shen, K.; Chiu, D. T. Single-synapse ablation and long-term imaging in live C. elegans. J. Neurosci. Methods 2008, 173, 20-26.

(251) Chang, T.-Y.; Pardo-Martin, C.; Allalou, A.; Wählby, C.; Yanik, M. F. Fully automated cellular-resolution vertebrate screening platform with parallel animal processing. Lab Chip 2012, 12, 711-716.

(252) Pardo-Martin, C.; Chang, T.-Y.; Koo, B. K.; Gilleland, C. L.; Wasserman, S. C.; Yanik, M. F. High-throughput in vivo vertebrate screening. Nat. Methods 2010, 7, 634636.

(253) Yamada, N.; Okano, T.; Sakai, H.; Karikusa, F.; Sawasaki, Y.; Sakurai, Y. Thermo-responsive polymeric surfaces; control of attachment and detachment of cultured cells. Wiley 1990.

(254) Okano, T.; Yamada, N.; Sakai, H.; Sakurai, Y. A novel recovery system for cultured cells using plasma-treated polystyrene dishes grafted with poly $(\mathrm{N}$ isopropylacrylamide). J. Biomed. Mater. Res. 1993, 27, 1243-1251.

(255) Edahiro, J.-I.; Sumaru, K.; Tada, Y.; Ohi, K.; Takagi, T.; Kameda, M.; Shinbo, T.; Kanamori, T.; Yoshimi, Y. In situ control of cell adhesion using photoresponsive culture surface. Biomacromolecules 2005, 6, 970-974.

(256) Inui, T.; Kurashina, Y.; Imashiro, C.; Takemura, K. Method of localized removal of cells using a bolt-clamped
Langevin transducer with an ultrasonic horn. Eng. Life Sci. 2019, 19, 575-583.

(257) Inui, T.; Mei, J.; Imashiro, C.; Kurashina, Y.; Friend, J.; Takemura, K. Focused surface acoustic wave locally removes cells from culture surface. Lab Chip 2021, 21, 1299-1306.

(258) Bulina, M. E.; Lukyanov, K. A.; Britanova, O. V.; Onichtchouk, D.; Lukyanov, S.; Chudakov, D. M. Chromophore-assisted light inactivation (CALI) using the phototoxic fluorescent protein KillerRed. Nat. Protoc. 2006, $1,947-953$.

(259) Xu, X.; Jia, J.; Guo, M. The Most Recent Advances in the Application of Nano-Structures/Nano-Materials for Single-Cell Sampling. Front. Chem. 2020, 8, 718.

(260) Actis, P.; Maalouf, M. M.; Kim, H. J.; Lohith, A.; Vilozny, B.; Seger, R. A.; Pourmand, N. Compartmental genomics in living cells revealed by single-cell nanobiopsy. ACS Nano 2014, 8, 546-553.

(261) Tan, S. C.; Yiap, B. C. DNA, RNA, and protein extraction: the past and the present. J. Biomed. Biotechnol. 2009, 2009, 574398.

(262) Gawad, C.; Koh, W.; Quake, S. R. Single-cell genome sequencing: current state of the science. Nat. Rev. Genet. 2016, 17, 175-188.

(263) Higgins, S. G.; Stevens, M. M. Extracting the contents of living cells. Science 2017, 356, 379-380.

(264) Frommer, W. B.; Davidson, M. W.; Campbell, R. E. Genetically encoded biosensors based on engineered fluorescent proteins. Chem. Soc. Rev. 2009, 38, 2833-2841.

(265) Jensen, E. C. Use of fluorescent probes: their effect on cell biology and limitations. Anat Rec (Hoboken) 2012, 295, 2031-2036.

(266) Swenson, E. S.; Price, J. G.; Brazelton, T.; Krause, D. S. Limitations of green fluorescent protein as a cell lineage marker. Stem Cells 2007, 25, 2593-2600.

(267) Tao, W.; Evans, B.-G.; Yao, J.; Cooper, S.; Cornetta, K.; Ballas, C. B.; Hangoc, G.; Broxmeyer, H. E. Enhanced green fluorescent protein is a nearly ideal long-term expression tracer for hematopoietic stem cells, whereas DsRed-express fluorescent protein is not. Stem Cells 2007, 25, 670-678.

(268) Li, Z.-H.; Peng, J.; Chen, H.-L. Bioconjugated quantum dots as fluorescent probes for biomedical imaging. $\mathrm{J} \mathrm{Na}$ nosci Nanotechnol 2011, 11, 7521-7536.

(269) Hiramoto, Y. An analysis of the mechanism of fertilization by means of enucleation of sea urchin eggs. Exp. Cell Res. 1962, 28, 323-334.

(270) Shakoor, A.; Xie, M.; Luo, T.; Hou, J.; Shen, Y.; Mills, J. K.; Sun, D. Achieving automated organelle biopsy on small single cells using a cell surgery robotic system. IEEE Trans. Biomed. Eng. 2019, 66, 2210-2222.

(271) Karrer, E. E.; Lincoln, J. E.; Hogenhout, S.; Bennett, A. B.; Bostock, R. M.; Martineau, B.; Lucas, W. J.; Gilchrist, D. G.; Alexander, D. In situ isolation of mRNA from individual plant cells: creation of cell-specific cDNA libraries. Proc. Natl. Acad. Sci. USA 1995, 92, 3814-3818.

(272) Tomos, A. D.; Sharrock, R. A. Cell sampling and analysis (SiCSA): metabolites measured at single cell resolution. $J$. Exp. Bot. 2001, 52, 623-630.

(273) Hofmann, P. A.; Hartzell, H. C.; Moss, R. L. Alterations in $\mathrm{Ca} 2+$ sensitive tension due to partial extraction of $\mathrm{C}$ protein from rat skinned cardiac myocytes and rabbit skeletal muscle fibers. J. Gen. Physiol. 1991, 97, 11411163.

(274) Stephens, D. J.; Pepperkok, R. The many ways to cross the plasma membrane. Proc. Natl. Acad. Sci. USA 2001, 98, 4295-4298.

(275) Liu, J.; Wen, J.; Zhang, Z.; Liu, H.; Sun, Y. Voyage inside the cell: Microsystems and nanoengineering for intracellular measurement and manipulation. Microsyst. Nanoeng. 2015, 1, 15020. 
(276) Guillaume-Gentil, O.; Potthoff, E; Ossola, D.; Franz, C. M.; Zambelli, T.; Vorholt, J. A. Force-controlled manipulation of single cells: from AFM to FluidFM. Trends Biotechnol. 2014, 32, 381-388.

(277) Nawarathna, D.; Chang, R.; Nelson, E.; Wickramasinghe, H. K. Targeted messenger RNA profiling of transfected breast cancer gene in a living cell. Anal. Biochem. 2011, 408, 342-344.

(278) Han, S.-W.; Nakamura, C.; Kotobuki, N.; Obataya, I.; Ohgushi, H.; Nagamune, T.; Miyake, J. High-efficiency DNA injection into a single human mesenchymal stem cell using a nanoneedle and atomic force microscopy. $\mathrm{Na}$ nomedicine 2008, 4, 215-225.

(279) Stanley, J.; Pourmand, N. Nanopipettes-The past and the present. APL Mater. 2020, 8, 100902.

(280) Bulbul, G.; Chaves, G.; Olivier, J.; Ozel, R. E.; Pourmand, N. Nanopipettes as monitoring probes for the single living cell: state of the art and future directions in molecular biology. Cells 2018, 7.

(281) Actis, P. Sampling from Single Cells. Small Methods 2018, 2, 1700300.

(282) Takami, T.; Park, B. H.; Kawai, T. Nanopipette exploring nanoworld. Nano Convergence 2014, 1, 17.

(283) Gao, R.; Cui, L.-F.; Ruan, L.-Q.; Ying, Y.-L.; Long, Y.-T. A Closed-Type Wireless Nanopore Electrode for Analyzing Single Nanoparticles. J. Vis. Exp. 2019.

(284) Pan, R.; Xu, M.; Burgess, J. D.; Jiang, D.; Chen, H.-Y. Direct electrochemical observation of glucosidase activity in isolated single lysosomes from a living cell. Proc. Natl. Acad. Sci. USA 2018, 115, 4087-4092.

(285) Clarke, R. W.; White, S. S.; Zhou, D.; Ying, L.; Klenerman, D. Trapping of Proteins under Physiological Conditions in a Nanopipette. Angew. Chem. Int. Ed. Engl. 2005, $117,3813-3816$

(286) Hao, R.; Zhang, B. Nanopipette-Based Electroplated Nanoelectrodes. Anal. Chem. 2016, 88, 614-620.

(287) Yin, R.; Prabhakaran, V.; Laskin, J. Electroosmotic extraction coupled to mass spectrometry analysis of metabolites in live cells. Meth. Enzymol. 2019, 628, 293-307.

(288) Qian, R.-C.; Lv, J.; Long, Y.-T. Ultrafast Mapping of Subcellular Domains via Nanopipette-Based Electroosmotically Modulated Delivery into a Single Living Cell. Anal. Chem. 2018, 90, 13744-13750.

(289) Adam Seger, R.; Actis, P.; Penfold, C.; Maalouf, M.; Vilozny, B.; Pourmand, N. Voltage controlled nanoinjection system for single-cell surgery. Nanoscale 2012, 4, 5843-5846.

(290) Chen, A.; Lynch, K. B.; Ren, J.; Jia, Z.; Yang, Y.; Lu, J. J.; Liu, S. Tunable Electroosmosis-Based Femto-Liter Pipette: A Promising Tool toward Living-Cell Surgery. Anal. Chem. 2017, 89, 10806-10812.

(291) Laforge, F. O.; Carpino, J.; Rotenberg, S. A.; Mirkin, M. V. Electrochemical attosyringe. Proc. Natl. Acad. Sci. USA 2007, 104, 11895-11900.

(292) Nashimoto, Y.; Takahashi, Y.; Zhou, Y.; Ito, H.; Ida, H.; Ino, K.; Matsue, T.; Shiku, H. Evaluation of mRNA Localization Using Double Barrel Scanning Ion Conductance Microscopy. ACS Nano 2016, 10, 6915-6922.

(293) Nashimoto, Y.; Echigo, M.; Ino, K.; Shiku, H. SiteSpecific Cytosol Sampling from a Single Cell in an Intact Tumor Spheroid Using an Electrochemical Syringe. Anal. Chem. 2019, 91, 8772-8776.

(294) Wang, H.-Y.; Ruan, Y.-F.; Zhu, L.-B.; Shi, X.-M.; Zhao, W.-W.; Chen, H.-Y.; Xu, J.-J. An integrated electrochemical nanodevice for intracellular RNA collection and detection in single living cell. Angew. Chem. Int. Ed. Engl. 2021, 60, 13244-13250.

(295) Bury, A. G.; Vincent, A. E.; Turnbull, D. M.; Actis, P.; Hudson, G. Mitochondrial isolation: when size matters. Wellcome Open Res. 2020, 5, 226.
(296) Burke, P. J. Nanodielectrophoresis: electronic nanotweezers. Encyclopedia of nanoscience and nanotechnology 2004, 6, 623-641.

(297) Cao, Y.; Hjort, M.; Chen, H.; Birey, F.; Leal-Ortiz, S. A.; Han, C. M.; Santiago, J. G.; Paşca, S. P.; Wu, J. C.; Melosh, N. A. Nondestructive nanostraw intracellular sampling for longitudinal cell monitoring. Proc. Natl. Acad. Sci. USA 2017, 114, E1866-E1874.

(298) He, G.; Yang, C.; Hang, T.; Liu, D.; Chen, H.-J.; Zhang, A.-H.; Lin, D.; Wu, J.; Yang, B.-R.; Xie, X. Hollow Nanoneedle-Electroporation System To Extract Intracellular Protein Repetitively and Nondestructively. ACS Sens. 2018, 3, 1675-1682.

(299) VanDersarl, J. J.; Xu, A. M.; Melosh, N. A. Nanostraws for direct fluidic intracellular access. Nano Lett. 2012, 12, 3881-3886.

(300) Xie, X.; Xu, A. M.; Leal-Ortiz, S.; Cao, Y.; Garner, C. C.; Melosh, N. A. Nanostraw-electroporation system for highly efficient intracellular delivery and transfection. ACS Nano 2013, 7, 4351-4358.

(301) Wen, R.; Zhang, A.-H.; Liu, D.; Feng, J.; Yang, J.; Xia, D.; Wang, J.; Li, C.; Zhang, T.; Hu, N.; et al. Intracellular delivery and sensing system based on electroplated conductive nanostraw arrays. ACS Appl. Mater. Interfaces 2019, 11, 43936-43948.

(302) Mukherjee, P.; Nathamgari, S. S. P.; Kessler, J. A.; Espinosa, H. D. Combined Numerical and Experimental Investigation of Localized Electroporation-Based Cell Transfection and Sampling. ACS Nano 2018, 12, 1211812128.

(303) Mukherjee, P.; Berns, E. J.; Patino, C. A.; Hakim Moully, E.; Chang, L.; Nathamgari, S. S. P.; Kessler, J. A.; Mrksich, M.; Espinosa, H. D. Temporal Sampling of Enzymes from Live Cells by Localized Electroporation and Quantification of Activity by SAMDI Mass Spectrometry. Small 2020, 16, e2000584.

(304) Meister, A.; Gabi, M.; Behr, P.; Studer, P.; Vörös, J.; Niedermann, P.; Bitterli, J.; Polesel-Maris, J.; Liley, M.; Heinzelmann, H.; et al. FluidFM: combining atomic force microscopy and nanofluidics in a universal liquid delivery system for single cell applications and beyond. Nano Lett. 2009, 9, 2501-2507.

(305) Guillaume-Gentil, O.; Grindberg, R. V.; Kooger, R.; Dorwling-Carter, L.; Martinez, V.; Ossola, D.; Pilhofer, M.; Zambelli, T.; Vorholt, J. A. Tunable Single-Cell Extraction for Molecular Analyses. Cell 2016, 166, 506-516.

(306) Amarouch, M. Y.; El Hilaly, J.; Mazouzi, D. AFM and fluidfm technologies: recent applications in molecular and cellular biology. Scanning 2018, 2018, 7801274.

(307) Lin, L.; Mawatari, K.; Morikawa, K.; Pihosh, Y.; Yoshizaki, A.; Kitamori, T. Micro/extended-nano sampling interface from a living single cell. Analyst 2017, 142, 16891696.

(308) Shakoor, A.; Luo, T.; Chen, S.; Xie, M.; Mills, J. K.; Sun, D. A high-precision robot-aided single-cell biopsy system. In 2017 IEEE International Conference on Robotics and Automation (ICRA); IEEE, 2017; pp. 5397-5402.

(309) Liu, R.; Pan, N.; Zhu, Y.; Yang, Z. T-Probe: An Integrated Microscale Device for Online In Situ Single Cell Analysis and Metabolic Profiling Using Mass Spectrometry. Anal. Chem. 2018, 90, 11078-11085.

(310) International review of cell and molecular biology; International review of cell and molecular biology; Elsevier, 2013; Vol. 304.

(311) Hindi, S. M.; Tajrishi, M. M.; Kumar, A. Signaling mechanisms in mammalian myoblast fusion. Sci. Signal. 2013, 6 , re2.

(312) Yagami-Hiromasa, T.; Sato, T.; Kurisaki, T.; Kamijo, K.; Nabeshima, Y.; Fujisawa-Sehara, A. A metalloproteasedisintegrin participating in myoblast fusion. Nature 1995, $377,652-656$. 
(313) Inoue, N.; Ikawa, M.; Isotani, A.; Okabe, M. The immunoglobulin superfamily protein Izumo is required for sperm to fuse with eggs. Nature 2005, 434, 234-238.

(314) Mohler, W. A.; Shemer, G.; del Campo, J. J.; Valansi, C.; Opoku-Serebuoh, E.; Scranton, V.; Assaf, N.; White, J. G.; Podbilewicz, B. The type I membrane protein EFF-1 is essential for developmental cell fusion. Dev. Cell 2002, $2,355-362$.

(315) Tamm, L. K. Protein-Lipid Interactions: From Membrane Domains to Cellular Networks; 1st ed.; Wiley-VCH: Weinheim, 2005; p. 470.

(316) Parray, H. A.; Shukla, S.; Samal, S.; Shrivastava, T.; Ahmed, S.; Sharma, C.; Kumar, R. Hybridoma technology a versatile method for isolation of monoclonal antibodies, its applicability across species, limitations, advancement and future perspectives. Int. Immunopharmacol. 2020, 85, 106639.

(317) Blau, H. M.; Chiu, C. P.; Webster, C. Cytoplasmic activation of human nuclear genes in stable heterocaryons. Cell 1983, 32, 1171-1180.

(318) Tada, M.; Tada, T.; Lefebvre, L.; Barton, S. C.; Surani, M. A. Embryonic germ cells induce epigenetic reprogramming of somatic nucleus in hybrid cells. EMBO J. 1997, 16, 6510-6520.

(319) Wilmut, I.; Schnieke, A. E.; McWhir, J.; Kind, A. J.; Campbell, K. H. Viable offspring derived from fetal and adult mammalian cells. Nature 1997, 385, 810-813.

(320) Cowan, C. A.; Atienza, J.; Melton, D. A.; Eggan, K. Nuclear reprogramming of somatic cells after fusion with human embryonic stem cells. Science 2005, 309, 1369 1373.

(321) Trefzer, U.; Walden, P. Hybrid-Cell Vaccines for Cancer Immune Therapy. $M B$ 2003, 25, 63-70.

(322) Okada, Y.; Tadokoro, J. Analysis of giant polynuclear cell formation caused by HVJ virus from Ehrlich's ascites tumor cells. Exp. Cell Res. 1962, 26, 108-118.

(323) Kao, K. N.; Michayluk, M. R. A method for highfrequency intergeneric fusion of plant protoplasts. Planta 1974, 115, 355-367.

(324) Pilwat, G.; Richter, H. P.; Zimmermann, U. Giant culture cells by electric field-induced fusion. FEBS Lett. 1981, 133, 169-174.

(325) Nilsson, J.; Evander, M.; Hammarström, B.; Laurell, T. Review of cell and particle trapping in microfluidic systems. Anal. Chim. Acta 2009, 649, 141-157.

(326) Skelley, A. M.; Kirak, O.; Suh, H.; Jaenisch, R.; Voldman, J. Microfluidic control of cell pairing and fusion. Nat. Methods 2009, 6, 147-152.

(327) Dura, B.; Liu, Y.; Voldman, J. Deformability-based microfluidic cell pairing and fusion. Lab Chip 2014, 14, 2783-2790.

(328) Wu, H.; Liu, W.; Tu, Q.; Song, N.; Li, L.; Wang, J.; Wang, J. Culture and chemical-induced fusion of tobacco mesophyll protoplasts in a microfluidic device. Microfluid. Nanofluidics 2011, 10, 867-876.

(329) Grabowski, M.; Buchenauer, A.; El Hasni, A.; Klockenbring, T.; Barth, S.; Mokwa, W.; Schnakenberg, U. Microfluidic system for cell fusion. Procedia Engineering 2010, 5, 1332-1335.

(330) Wang, J.; Lu, C. Microfluidic cell fusion under continuous direct current voltage. Appl. Phys. Lett. 2006, 89, 234102.

(331) Gel, M.; Suzuki, S.; Kimura, Y.; Kurosawa, O.; Techaumnat, B.; Oana, H.; Washizu, M. Microorificebased high-yield cell fusion on microfluidic chip: electrofusion of selected pairs and fusant viability. IEEE Trans Nanobioscience 2009, 8, 300-305.

(332) Gel, M.; Kimura, Y.; Kurosawa, O.; Oana, H.; Kotera, H.; Washizu, M. Dielectrophoretic cell trapping and parallel one-to-one fusion based on field constriction created by a micro-orifice array. Biomicrofluidics 2010, 4 .
(333) Kimura, Y.; Gel, M.; Techaumnat, B.; Oana, H.; Kotera, H.; Washizu, M. Dielectrophoresis-assisted massively parallel cell pairing and fusion based on field constriction created by a micro-orifice array sheet. Electrophoresis 2011, 32, 2496-2501.

(334) Wada, K.-I.; Hosokawa, K.; Ito, Y.; Maeda, M. A novel cell fusion method for direct cytoplasmic transfer using a microfluidic device. In Advances in bioinspired and biomedical materials volume 2; Ito, Y.; Chen, X.; Kang, I.K., Eds.; ACS Symposium Series; American Chemical Society: Washington, DC, 2017; Vol. 1253, pp. 227-236.

(335) Cao, Y.; Yang, J.; Yin, Z. Q.; Luo, H. Y.; Yang, M.; Hu, N.; Yang, J.; Huo, D. Q.; Hou, C. J.; Jiang, Z. Z.; et al. Study of high-throughput cell electrofusion in a microelectrode-array chip. Microfluid. Nanofluidics 2008, 5, 669-675.

(336) Qu, Y.; Hu, N.; Xu, H.; Yang, J.; Xia, B.; Zheng, X.; Yin, Z. Q. Somatic and stem cell pairing and fusion using a microfluidic array device. Microfluid. Nanofluidics 2011, $11,633-641$.

(337) Hu, N.; Yang, J.; Yin, Z.-Q.; Ai, Y.; Qian, S.; Svir, I. B.; Xia, B.; Yan, J.-W.; Hou, W.-S.; Zheng, X.-L. A highthroughput dielectrophoresis-based cell electrofusion microfluidic device. Electrophoresis 2011, 32, 2488-2495.

(338) Hu, N.; Yang, J.; Qian, S.; Joo, S. W.; Zheng, X. A cell electrofusion microfluidic device integrated with 3D thinfilm microelectrode arrays. Biomicrofluidics 2011, 5, 34121-3412112.

(339) Lu, Y.-T.; Pendharkar, G. P.; Lu, C.-H.; Chang, C.-M.; Liu, C.-H. A microfluidic approach towards hybridoma generation for cancer immunotherapy. Oncotarget 2015, $6,38764-38776$.

(340) Kandušer, M.; Ušaj, M. Cell electrofusion: past and future perspectives for antibody production and cancer cell vaccines. Expert Opin Drug Deliv 2014, 11, 1885-1898.

(341) Kato, M.; Sasamori, E.; Chiba, T.; Hanyu, Y. Cell activation by CpG ODN leads to improved electrofusion in hybridoma production. J. Immunol. Methods 2011, 373, $102-110$.

(342) Usaj, M.; Kanduser, M. The systematic study of the electroporation and electrofusion of B16-F1 and $\mathrm{CHO}$ cells in isotonic and hypotonic buffer. J. Membr. Biol. 2012, 245, 583-590.

(343) Usaj, M.; Trontelj, K.; Miklavcic, D.; Kanduser, M. Cellcell electrofusion: optimization of electric field amplitude and hypotonic treatment for mouse melanoma (B16-F1) and Chinese Hamster ovary (CHO) cells. J. Membr. Biol. 2010, 236, 107-116.

(344) Rems, L.; Ušaj, M.; Kandušer, M.; Reberšek, M.; Miklavčič, D.; Pucihar, G. Cell electrofusion using nanosecond electric pulses. Sci. Rep. 2013, 3, 3382.

(345) Schoeman, R. M.; van den Beld, W. T. E.; Kemna, E. W. M.; Wolbers, F.; Eijkel, J. C. T.; van den Berg, A. Electrofusion of single cells in picoliter droplets. Sci. Rep. 2018, 8, 3714.

(346) Yoshimura, Y.; Tomita, M.; Mizutani, F.; Yasukawa, T. Cell pairing using microwell array electrodes based on dielectrophoresis. Anal. Chem. 2014, 86, 6818-6822.

(347) He, W.; Huang, L.; Feng, Y.; Liang, F.; Ding, W.; Wang, W. Highly integrated microfluidic device for cell pairing, fusion and culture. Biomicrofluidics 2019, 13, 054109.

(348) Kaneda, Y.; Nakajima, T.; Nishikawa, T.; Yamamoto, S.; Ikegami, H.; Suzuki, N.; Nakamura, H.; Morishita, R.; Kotani, H. Hemagglutinating virus of Japan (HVJ) envelope vector as a versatile gene delivery system. Mol. Ther. 2002, 6, 219-226.

(349) Sasaki, N.; Gong, J.; Sakuragi, M.; Hosokawa, K.; Maeda, M.; Ito, Y. Hydrodynamic Cell Pairing and Cell Fusion through a Microslit on a Microfluidic Device. Jpn. J. Appl. Phys. 2012, 51, 030206.

(350) Tocchio, A.; Durmus, N. G.; Sridhar, K.; Mani, V.; Coskun, B.; El Assal, R.; Demirci, U. Magnetically Guid- 
ed Self-Assembly and Coding of 3D Living Architectures. Adv. Mater. Weinheim 2018, 30.

(351) Tomasi, R. F.-X.; Sart, S.; Champetier, T.; Baroud, C. N. Individual Control and Quantification of 3D Spheroids in a High-Density Microfluidic Droplet Array. Cell Rep. 2020, 31, 107670.

(352) Zhao, L.; Liu, Y.; Liu, Y.; Zhang, M.; Zhang, X. Microfluidic Control of Tumor and Stromal Cell Spheroids Pairing and Merging for Three-Dimensional Metastasis Study. Anal. Chem. 2020, 92, 7638-7645.

(353) Weisz, P. B. Homoplastic grafting in stentor coeruleus. Biol Bull 1951, 100, 116-126.

(354) Tartar, V. Grafting experiments concerning primordium formation in Stentor coeruleus. J. Exp. Zool. 1956, 131, 75-121.

(355) Voskoboynik, A.; Weissman, I. L. Botryllus schlosseri, an emerging model for the study of aging, stem cells, and mechanisms of regeneration. Invertebr. Reprod. Dev. 2015, 59, 33-38.

(356) Bely, A. E.; Nyberg, K. G. Evolution of animal regeneration: re-emergence of a field. Trends Ecol. Evol. (Amst.) 2010, 25, 161-170.

(357) Sánchez Alvarado, A.; Tsonis, P. A. Bridging the regeneration gap: genetic insights from diverse animal models. Nat. Rev. Genet. 2006, 7, 873-884.

(358) Rozenblatt-Rosen, O.; Stubbington, M. J. T.; Regev, A.; Teichmann, S. A. The Human Cell Atlas: from vision to reality. Nature 2017, 550, 451-453.

(359) Mandal, A.; Boopathy, A. V.; Lam, L. K. W.; Moynihan, K. D.; Welch, M. E.; Bennett, N. R.; Turvey, M. E.; Thai, N.; Van, J. H.; Love, J. C.; et al. Cell and fluid sampling microneedle patches for monitoring skin-resident immunity. Sci. Transl. Med. 2018, 10.

(360) Jin, Q.; Yang, Y.; Jackson, J. A.; Yoon, C.; Gracias, D. H. Untethered single cell grippers for active biopsy. Nano Lett. 2020, 20, 5383-5390.

(361) Gao, R. Z.; Ren, C. L. Synergizing microfluidics with soft robotics: A perspective on miniaturization and future directions. Biomicrofluidics 2021, 15, 011302. 